\title{
A von Neumann Algebra Framework for the Duality of the Quantum Groups
}

\author{
Dedicated to Professor Masamichi Takesaki on his 60th birthday
}

By

Tetsuya MASUDA $^{*}$ and Yoshiomi NAKAGAMI ${ }^{* *}$

\section{§o. Introduction}

Classical Lie groups are important examples in the category of locally compact groups. The general theory of unitary representations is developed for these objects as harmonic analysis, which provides us a good theoretical framework for the detailed study of the unitary representations of the classical Lie groups. This is regarded as an extension of the Fourier analysis to a general context. For a locally compact group, its dual i.e. the set of all the equivalence classes of irreducible unitary representations plays an important role, and the duality established by Pontrjagin for Abelian groups, Tannaka and Krein for compact groups, Steinspring for unimodular groups, Eymard and Tatsuuma for locally compact groups is an important theoretical basis for the harmonic analysis. On the other hand, at the formal level in the framework of pure algebras, we use the notion of Hopf algebras to deal with the algebraic groups, discrete groups, or the dual of those objects at the same time. Then functional analysis is necessarily combined with the algebraic framework of Hopf algebras to have a good control with the infinite dimensional unitary representations. This theory, especially the argument utilized by Steinspring, suggests us to introduce the notion of Kac algebras in the language of von Neumann algebras. The first take off from the group or the group algebra to the Kac algebra was considered by Kac [7] and performed by Takesaki [23] by introducing the, so-called, Kac-Takesaki operator or the fundamental operator for the semifinite i.e. the unimodular case, and then completed by Enock and Schwartz $[4,20,5]$ for the general case, in which the above mentioned duality was established by Takesaki, Enock and Schwartz, and others [24, 21].

Communicated by H. Araki, October 19, 1993.

1991 Mathematics Subject Classifications : 46L10, 81R50, 16W30

* Institute of Mathematics, University of Tsukuba, Tsukuba, Ibaraki, 305 Japan.

** Department of Mathematics, Yokohama City University, Seto, Kanazawa-ku, Yokohama, 236 Japan. 
In the recent study of the quantum inverse scattering method, non-commutative and non-cocommutative Hopf algebras were introduced in $[6,3]$, as a natural " $q$ deformation" or " $q$-analogue" of the universal enveloping algebra of simisimple Lie algebras and called by name "quantum groups". Around the same time, Woronowicz [26] also discovered independently an object as a $C^{*}$-algebra which looks like a noncommutative deformation of the Hopf algebra of complex valued continuous functions on the compact Lie group $S U(2)$. It then turned out that these objects were essentially the same. (See [18], for example.)

At the present stage, the "quantum groups" are known by these explicitly given examples for which the finite dimensional representation theory is developed. As an object of pure algebra, "quantum group" makes sense as a Hopf algebra with no assumptions on its commutativity or co-commutativity. However, if we take the complex number field $\mathbb{C}$ as the coefficient field of these particular Hopf algebras as the coordinate rings of corresponding "quantum groups", we are able to specify the *structures and the unitarity of the representations make sense with which we are allowed to manage the infinite dimensional representations in some explicit cases.

Those success in the representation theory are considered to be still formal. However, these are the good evidences to believe the existence of a suitable category which naturally contains not only group duals but also "quantum groups". Up to the present stage, nobody seemed to have already defined some good frameworks to deal with the infinite dimensional representations of these "quantum groups" in a rigorous way. The purpose of this paper is to develop an abstract framework of harmonic analysis which works also for these "quantum groups" including the non-compact cases (see [17] for example). In this paper, we use the language of von Neumann algebras to formulate the category in which we are able to deal with the "quantum groups" and prove that the duality holds.

We now describe the arrangements of this paper as follows. In Section 1, we define a Woronowicz algebra $(M, \delta, R, \tau, h)$ by introducing a deformation automorphism $\left\{\tau_{t}\right\}$ into a Kac algebra, where $M$ is a von Neumann algebra, $\delta$ is a coassociative coproduct, $R$ is a unitary antipode and $h$ is a left invariant Haar weight. When the Woronowicz algebra is commutative or finite dimensional, it goes back to a Kac algebra. According to the succeeding discussion, this concept will be recognized as a natural object of the von Neumann algebra version of quantum groups. In Section 2 we construct a left Hilbert algebra coming from the predual $M_{*}$ of the Woronowicz algebra. We also show the unitarity of the Kac-Takesaki operator $W$, whose adjoint will play the role of the left regular representation. The unitarity is also equivalent to the density of the linear span of quadratic elements of the Hilbert algebra. In our subsequent argument, the commutativity of four positive selfadjoint operators, the modular operator $\Delta$ with respect to the Haar weight, the deformation operator $H$ implementing the deformation automorphism and the module operators $\rho, J \rho J$ of the Haar weight is extensively used. Utilizing the Fourier transform $\hat{\pi}(\phi)=(\phi \otimes \mathrm{id})\left(W^{*}\right)$ for $\phi \in M_{*}$, we construct the dual Woronowicz algebra in Section 3. Our Section 4 is devoted to the proof of the 
general duality theorem. Finally, in Section 5, we discuss, as an example, a Woronowicz algebra which corresponds to the quantum group $S U_{q}(n)$.

We have to remark that the complex number field $\mathbf{C}$ is not the only possible choice for the coefficient field of the coordinate rings of the "quantum groups". However, the global geometrical nature of the Lie groups are very well described by the unitarity of the representations which play important roles in the theory of locally compact groups. This is why we are more or less obliged to use the language of operator algebras which is not apriori automatic. It also has to be mentioned that, for the purpose of obtaining a reasonable framework in this direction, the language of $C^{*}$-algebras should be used to formulate the category which corresponds to the category of locally compact topological groups. Indeed, Woronowicz had also studied the same type of problem for compact quantum groups by using the "polar decomposition" of the antipode mapping as we will discuss in terms of the unitary antipode and the deformation automorphism. However, for the purpose of avoiding some technicalities in the full algebraic generality, we use the language of von Neumann algebras in the present publication. It is desirable to discuss the framework using the language of $C^{*}$-algebras, which will be our future subject.

The primitive ideas of this publication were already presented in [9]. This publication is a detailed version of our previous talk [11]. The crossed product in our context was also developed in [16].

\section{§1. General Framework}

We start by clarifying our situation in which we deal with the unitary representations of "quantum groups" using the language of von Neumann algebras. Since our motivation comes from the "quantum groups", we have an essential difficulty which is the unboundedness of the antipode mapping of our "involutive Hopf algebra". This is a difficulty which is apparent even in the "simplest case" $S U_{q}(2)$. Algebraically, this corresponds to the non-triviality of the automorphism of the Hopf algebra obtained by the square of the antipode mapping. This difficulty is not particular for the framework using von Neumann algebras which still exists even in the $C^{*}$-algebraic framework. Therefore, this point is considered to be one of the essential features of the new category in which we are able to deal with the "quantum groups". So, for the purpose of formulating our category in terms of operator algebras, we introduce an auxiliary structure i.e. one parameter family of $*$-automorphisms which we call deformation automorphism. It is also remarked that, in the classical situation, the unit element is negligible with respect to the Haar measure. (This is one of the disadvantages to use the language of von Neumann algebras.) Therefore we have to give up using the counit of our "involutive Hopf algebra" in our framework. The axioms for the counit and the left invariant Haar measure (Haar weight) are combined and replaced by a condition which we call the strong left invariance. This condition technically simplifies our formulation as in the case for the framework of Kac algebra. 
First of all, we remark on our basic notational convention of this paper that all the tensor products of the von Neumann algebras are the spacial tensor products which we denote simply by $\otimes$. Then, all the tensor products of the Hilbert spaces are also denoted by the same symbol $\otimes$ with which we have no danger of confusions. The set of all bounded operators on the Hilbert space $\mathfrak{S}$ is denoted by $\mathscr{L}(\mathfrak{S})$. We also give an obvious remark that we use the usual notions of Tomita-Takesaki theory. For example,

$$
\mathfrak{n}_{h}=\left\{x \in M: h\left(x^{*} x\right)<\infty\right\} .
$$

We also use the notation $x \in \mathfrak{n}_{h} \rightarrow \eta_{h}(x) \in \mathfrak{S}_{h}$ which identifies an element of $\mathfrak{n}_{h}$ with the element of the Hilbert space $\mathfrak{S}_{h}$ which is given by the GNS construction using $h$. For $x \in M$, we denote by $\pi_{h}(x)$ the corresponding left multiplication operator acting on the Hilbert space $\mathfrak{S}_{h}$ given by

$$
\pi_{h}(x) \eta_{h}(y)=\eta_{h}(x y) \text { for } y \in \mathfrak{n}_{h} .
$$

Furthermore, the left involution operator obtained as the closure of the conjugate linear mapping : $\eta_{h}(x) \rightarrow \eta_{h}\left(x^{*}\right)$ and its polar decomposition are denoted by $S$ and $J \Delta^{1 / 2}$, where the modular conjugation operator $J\left(=J_{h}\right)$ is conjugate unitary and involutive, and the modular operator $\Delta\left(=\Delta_{h}\right)$ is positive and self-adjoint. The adjoint $F$ of $S$ is the right involution operator and the polar decomposition is given by $J \Delta^{-1 / 2}=\Delta^{1 / 2} J$. The modular automorphism group $\left\{\sigma_{t}^{h}\right\}$ is given by $\sigma_{t}^{h}(x)=\Delta^{t t} x \Delta^{-i t}$ for $x \in M$. For these notations, we refer to the standard reference [22] of Takesaki.

Now, our basic definition which we introduce in this section is given by the following.

Definition 1.1. The family $(M, \delta, R, \tau, h)$ with the following conditions is called a Woronowicz algebra:

(i) $M$ is a von Neumann algebra with a comultiplication $\delta: M \rightarrow M \otimes M$ which is a normal $*$-isomorphism with the coassociativity $(\delta \otimes$ id $) \circ \delta=($ id $\otimes \delta) \circ \delta$.

(ii) Unitary antipode is a *-antiautomorphism $R: M \rightarrow M$ such that $R^{2}=$ id and $\sigma \circ(R \otimes R) \circ \delta=\delta \circ R$, where $\sigma: M \otimes M \rightarrow M \otimes M, x \otimes y \rightarrow y \otimes x$ is the flip mapping.

(iii) Deformation automorphism is a continuous one parameter group $\left\{\tau_{t}\right\}$ of $*$ automorphisms $\tau: \mathbf{R} \rightarrow \operatorname{Aut}(M)$ such that the mappings $\delta$ and $R$ are invariant i.e. $\left(\tau_{t} \otimes \tau_{t}\right) \circ \delta=\delta \circ \tau_{t}$ and $\tau_{t} \circ R=R \circ \tau_{t}$ for $t \in \mathbf{R}$.

(iv) (left invariant) Haar weight $h$ is a $\left\{\tau_{t}\right\}$-invariant semifinite faithful normal weight on the von Neumann algebra $M$ such that the following conditions hold:

a) Left invariance: For any positive $\phi \in M_{*}$, the weight $(\phi \otimes h) \circ \delta$ coincides with the weight $\phi(1) h$.

b) Strong left invariance: For any $x, y \in \mathfrak{n}_{h}$, and $\phi \in M_{*}$ which is entire analytic with respect to the action of the deformation automorphism $\left\{\tau_{t}\right\}$ on the predual $M_{*}$, the following equality holds:

$$
(\phi \otimes h)\left(\left(1 \otimes y^{*}\right) \delta(x)\right)=\left(\phi \circ \tau_{-t / 2} \circ R \otimes h\right)\left(\delta\left(y^{*}\right)(1 \otimes x)\right) .
$$


c) Commutativity: The two weights $h$ and $h \circ R$ commute (i.e. the corresponding modular automorphisms commute).

We remark that for any positive $\phi \in M_{*}$ satisfying $\phi(1)=1$, the mapping $\phi \otimes \mathrm{id}: M \otimes M \rightarrow M$ defined by $x \otimes y \rightarrow \phi(x) y$ is a conditional expectation under the identification $M \rightarrow M \otimes M$ by $x \rightarrow 1 \otimes x$. Therefore we have $(\phi \otimes \mathrm{id})(\delta(x))^{*}(\phi \otimes \mathrm{id})(\delta(x)) \leq(\phi \otimes \mathrm{id})\left(\delta\left(x^{*} x\right)\right)$ for $x \in M$. This inequality combined with the left invariance of the Haar weight implies $(\phi \otimes$ id $)\left(\delta\left(\mathfrak{n}_{h}\right)\right) \subset \mathfrak{n}_{h}$ for any $\phi \in M_{*}$. This inclusion relation guarantees that the both sides of the equality in the above strong left invariance are finite.

By the commutativity of the weights $h$ and $h \circ R$, there exists a positive selfadjoint element $\rho$ affiliated with $M$ such that the Radon-Nikodym cocycle satisfies $(D h \circ R: D h)_{t}=\rho^{-t t}$ for $t \in \mathbf{R}$. Since $R=R^{-1}$, it satisfies

$$
R\left(\rho^{t t}\right)=R\left((D h \circ R: D h)_{-t}\right)=(D h: D h \circ R)_{-t}=\rho^{-t t} .
$$

Remark 1.2. An involutive Hopf algebra $\left(A, \mu_{A}, \delta_{A}, \varepsilon_{A}, \kappa_{A}\right)$ is a Hopf algebra with the following additional conditions: (i) $A$ is an involutive unital algebra with respect to the multiplication $\mu_{A}: a \otimes b \in A \otimes A \rightarrow a b \in A$; (ii) the comultiplication $\delta_{A}$ and the counit $\varepsilon_{A}$ preserve the involution. Then it is known that the antipode $\kappa_{A}$ satisfies the Woronowicz condition $\kappa_{A}\left(a^{*}\right)=\kappa_{A}^{-1}(a)^{*}$ for $a \in A$ and

$$
\sigma \circ\left(\kappa_{A} \otimes \kappa_{A}\right) \circ \delta_{A}=\delta_{A} \circ \kappa_{A} .
$$

Further, if the involutive Hopf algebra has a Haar state $h$, i.e. if $h \in A^{*}$ is a positive linear functional which satisfies the normalization condition $h(1)=1$ and the left invariance $(\mathrm{id} \otimes h)\left(\delta_{A}(a)\right)=h(a) 1$, then the strong left invariance

$$
(\mathrm{id} \otimes h)\left(\left(1 \otimes b^{*}\right) \delta_{A}(a)\right)=\left(\kappa_{A} \otimes h\right)\left(\delta_{A}\left(b^{*}\right)(1 \otimes a)\right)
$$

automatically holds. Moreover, $h=h \circ \kappa_{A}$, because $h \circ \kappa_{A}$ is right invariant by (1.2) and hence $h \circ \kappa_{A}(a) h(1)=\left(h \circ \kappa_{A} \otimes h\right)\left(\delta_{A}(a)\right)=h(1) h(a)$. The left and the right invariance of the Haar state yields the uniqueness of a Haar state: $h_{1}(a) h(1)$ $=\left(h_{1} \otimes h\right)\left(\delta_{A}(a)\right)=h_{1}(1) h(a)$ if $h_{1}$ is another Haar state.

Remark 1.3. a) It should be mentioned that the antipode mapping of the involutive Hopf algebra associated with the "quantum group" corresponds to the formal expression $R \circ \tau_{-l / 2}=\tau_{-t / 2} \circ R$. Furthermore, if $\tau$ is trivial, our situation reduces to that of Kac algebras.

b) When the Haar weight $h$ is bounded i.e. it satisfies $h(1)<\infty$, the strong left invariance implies the left invariance of $h$ by putting $y=1$. The normalized bounded Haar weight is sometimes called the Haar state.

c) The weight $h \circ R$ is right invariant: $(h \circ R \otimes \phi)(\delta(z))=h \circ R(z) \phi(1)$ for $\phi \in M_{*}$ by the property of the unitary antipode. If $h$ is bounded, then $h=h \circ R$ (or $\rho=1$ ) and 
the uniqueness of the Haar weight up to scalar is deduced from its bi-invariance property as above. The uniqueness for an unbounded case is also proved in Remark 4.7.

d) If the von Neumann algebra for a Woronowicz algebra is finite dimensional or commutative, then the deformation automorphism turns out to be trivial and hence the Woronowicz algebra reduces to a Kac algebra as shown in Remark 3.18. In addition, in the finite dimensional case, the concepts of a Woronowicz algebra, a Kac algebra and an involutive Hopf algebra are the same as shown in Proposition 4.6. Furthermore, a commutative Woronowicz algebra is nothing but a Kac algebra defined by a locally compact group by [23].

In this paper, we will introduce several kinds of left Hilbert algebras as listed below:

1) left Hilbert algebras associated with $(M, h)$

$\mathfrak{A}_{h}=\eta_{h}\left(\mathfrak{a}_{h}\right)$; the achieved left Hilbert algebra

$\mathfrak{a}_{0}=\eta_{h}\left(\mathfrak{a}_{0}\right) ; \mathfrak{a}_{0}$ is the entire analytic part of $\mathfrak{a}_{h}$ with respect to four continuous transformations $\left\{\sigma_{t}^{h}\right\},\left\{\sigma_{t}^{h \circ R}\right\},\left\{\tau_{t}\right\}$ and $\left\{L_{t}\right\}$ (see the beginning of Section 2 for the definition of $\left.\left\{L_{t}\right\}\right)$

2) left Hilbert algebras associated with $(\hat{M}, \hat{h})$

$\mathfrak{A}_{\hat{h}}=\eta_{\hat{h}}\left(\mathfrak{a}_{\hat{h}}\right) ;$ the achieved left Hilbert algebra

$\hat{\mathfrak{U}}=\hat{\eta}(\mathfrak{b}) ;$ see Definition 2.3

$\hat{\mathfrak{A}}_{0}=\eta_{\hat{h}}\left(\hat{\mathfrak{a}}_{0}\right) ; \hat{\mathfrak{a}}_{0}$ is the entire analytic part of $\mathfrak{a}_{\hat{h}}$ with respect to the corresponding four continuous transformations

3) left Hilbert algebras associated with $(\hat{\hat{M}}, \hat{\hat{h}})$

$\mathfrak{U}_{\hat{\hat{h}}}=\eta_{\hat{\hat{h}}}\left(\mathfrak{a}_{\hat{\hat{h}}}\right) ;$ the achieved left Hilbert algebra

$\hat{\hat{\mathfrak{U}}}=\hat{\hat{\eta}}(\hat{\mathfrak{b}}) ;$ see Definition 2.3

4) left Hilbert algebras associated with the co-opposite Woronowicz algebra $\mathfrak{U}_{h^{\circ}}=\eta_{h^{\circ}}\left(\mathfrak{a}_{h^{\circ}}\right)$; the achieved left Hilbert algebra

$\hat{\mathfrak{U}}^{\circ}=\hat{\eta}^{\circ}\left(\mathfrak{b}^{\circ}\right)$; see Definition 2.3

5) left Hilbert algebras associated with the commutant Woronowicz algebra $\mathfrak{A}_{h^{\prime}}=\eta_{h^{\prime}}\left(\mathfrak{a}_{h^{\prime}}\right)$; the achieved left Hilbert algebra

\section{§2. Left Hilbert Algebra and Kac-Takesaki Operator}

In this section, we discuss some properties of the Kac-Takesaki operator to construct a left Hilbert algebra which we use in the subsequent section. If we deal with the purely algebraic Hopf algebra $A$ over $\mathbb{C}$, the dual Hopf algebra is constructed inside the linear dual $\operatorname{Hom}(A, \mathbb{C})$ of $A$ under some technical conditions. However, since we deal with a framework which involves functional analysis, we need an appropriate framework for the construction of the dual. 
For the purpose of constructing the dual object, we take a nice subspace of the predual $M_{*}$ of the original Woronowicz algebra to start with. Our discussion is based on the standard representation Hilbert space $\mathfrak{S}$ of $M$ associated with the Haar weight $h$ which is a semifinite faithful normal weight on $M$. Therefore $\{M, \mathfrak{g}\}$ is identified with $\left\{\pi_{h}(M), \mathfrak{S}_{h}\right\}$. The construction of our dual Woronowicz algebra $(\hat{M}, \hat{\delta}, \hat{R}, \hat{\tau}, \hat{h})$ is such that the Hilbert space $\mathbf{S}$ is also the standard representation Hilbert space of $\hat{M}$ associated with the dual Haar weight (Plancherel weight) $\hat{h}$ which is a semifinite faithful normal weight on $\hat{M}$.

The predual $M_{*}$ is endowed with a multiplication structure dual to the coproduct defined on $M$. Namely, the product $\phi * \psi \in M_{*}$ for $\phi, \psi$ in $M_{*}$ is defined by

$$
\left(\phi^{*} \psi\right)(x)=(\phi \otimes \psi)(\delta(x)), \quad x \in M .
$$

This product is associative due to the coassociativity of the coproduct. Thus $M_{*}$ is a Banach algebra. We denote by $\left(M_{*}\right)_{\tau}$ the set of all entire analytic elements in $M_{*}$ with respect to the action $\phi \rightarrow \phi \circ \tau_{t}$ of the deformation automorphism $\left\{\tau_{t}\right\}$ on the predual $M_{*}$. Then for $\phi \in\left(M_{*}\right)_{\tau}$, the involution $\phi^{\#} \in\left(M_{*}\right)_{\tau}$ is defined by $\phi^{\#}(x)=$ $\phi^{*}\left(\tau_{-l / 2} \circ R(x)\right), x \in M$, where $\phi^{*}$ denotes the linear functional on $M$ with $\phi^{*}(y)=$ $\overline{\phi\left(y^{*}\right)}$ and $\phi^{*} \circ \tau_{\bar{\alpha}}=\left(\phi \circ \tau_{\alpha}\right)^{*}$ for $\alpha \in \mathbf{C}$. This involution, combined with the above associative product, makes the set $\left(M_{*}\right)_{\tau}$ an involutive algebra.

We next define the $L^{2}$-boundedness for an element in the predual $M_{*}$.

Definition 2.1. An element $\phi \in M_{*}$ is said to be $L^{2}$-bounded if there exists a constant $\lambda \geq 0$ such that

$$
\left|\phi\left(y^{*}\right)\right| \leq \lambda\left\|\eta_{h}(y)\right\| \text { for } y \in \mathfrak{n}_{h} .
$$

We denote by $\hat{\eta}(\phi)$ the unique element in $\mathfrak{g}$ satisfying $\phi\left(y^{*}\right)=\left(\hat{\eta}(\phi) \mid \eta_{h}(y)\right)$ for $y \in \mathfrak{n}_{h}$.

The mapping $\hat{\eta}$ is linear and injective. Since $\mathfrak{B}$ is the standard representation Hilbert space of the von Neumann algebra $M$, any element in $M_{*}$ is of the form

$$
\omega_{\xi, \eta}(x)=(x \xi \mid \eta), x \in M, \xi, \eta \in \mathfrak{g} .
$$

In addition to this standard notational convention, we also use the same type of notations. Namely, in this paper, for the purpose of the notational simplification, we adapt the convention of writing $x$ or $\phi$ instead of $\eta_{h}(x)$ or $\hat{\eta}(\phi)$, respectively only in the case that these elements are put as the subscripts of $\omega$. For example we write $\omega_{\phi, i} \in M_{*}$ for $L^{2}$-bounded $\phi \in M_{*}$ and $x \in \mathfrak{n}_{h}$ to express a linear functional defined by

$$
\omega_{\phi, \lambda}(y)=\left(y \hat{\eta}(\phi) \mid \eta_{h}(x)\right), y \in M .
$$

Due to the conditions described in the axioms for the Woronowicz algebra, the one parameter group of unitaries $\left\{\rho^{t t}: t \in \mathbf{R}\right\}$ in $M$ are invariant under the actions of the 
modular automorphisms $\left\{\sigma_{t}^{h}\right\},\left\{\sigma_{t}^{h \circ R}\right\}$ as well as the deformation automorphisms $\left\{\tau_{t}\right\}$. We also introduce a one parameter family of linear transformations $\left\{L_{t}\right\}$ on $M$ defined by $L_{t}(x)=x \rho^{t t}, t \in \mathbf{R}$ and $x \in M$. Then $\rho^{t t} x \rho^{-t t}=\sigma_{t}^{h}\left(\sigma_{-t}^{h \circ R}(x)\right)$ for $t \in \mathbf{R}, x \in M$ and hence the four weakly continuous linear transformations $\left\{\sigma_{t}^{h}\right\},\left\{\sigma_{t}^{h \circ R}\right\},\left\{\tau_{t}\right\}$ and $\left\{L_{t}\right\}$ are commutative with each other, and the set $\mathfrak{a}_{h}=\left\{x \in \mathfrak{n}_{h}: x^{*} \in \mathfrak{n}_{h}\right\}$ is invariant under these four transformations. We denote by $a_{0}$ the set of all entire analytic elements in $\mathfrak{a}_{h}$ with respect to these four transformations. Then $\mathfrak{a}_{0}$ is also invariant under the actions of the unitary antipode $R$ as well as these four linear transformations. The analytic continuations of these four transformations preserve not only the $*$-algebra structure of $\mathfrak{a}_{0}$ but also the additive group structure of $\mathbf{C}$ on $\mathfrak{a}_{0}$. For example, for each $x, y \in \mathfrak{a}_{0}$ and $\alpha, \alpha_{1}, \alpha_{2} \in \mathbf{C}$,

$$
\begin{array}{ll}
\sigma_{\alpha}^{h}(x+y)=\sigma_{\alpha}^{h}(x)+\sigma_{\alpha}^{h}(y), & \sigma_{\alpha}^{h}(\lambda x)=\lambda \sigma_{\alpha}^{h}(x) \\
\sigma_{\alpha}^{h}(x y)=\sigma_{\alpha}^{h}(x) \sigma_{\alpha}^{h}(y), & \sigma_{\alpha}^{h}(x)^{*}=\sigma_{\bar{\alpha}}^{h}\left(x^{*}\right)
\end{array}
$$

and

$$
\sigma_{\alpha_{1}}^{h}\left(\sigma_{\alpha_{2}}^{h}(x)\right)=\sigma_{\alpha_{1}+\alpha_{2}}^{h}(x) .
$$

However $L_{\alpha}\left(x^{*}\right)=\left(L_{\bar{\alpha}} \circ \sigma_{\bar{\alpha}}^{h \circ R} \circ \sigma_{-\bar{\alpha}}^{h}(x)\right)^{*}$ for $x \in \mathfrak{a}_{0}$. If $\phi \in M_{*}$ is entire analytic with respect to $\left\{\tau_{t}\right\}$, then $\left(\phi \circ \tau_{\alpha}\right)(x)=\phi\left(\tau_{\alpha}(x)\right)$ for $\alpha \in \mathbf{C}$ and $x \in \mathfrak{a}_{0}$ by the unicity of the analytic continuation.

We see that the linear functional $\omega_{x, y} \in M_{*}$ for $x, y \in a_{0}$ is $L^{2}$-bounded and the vector $\hat{\eta}\left(\omega_{x, y}\right)$ is given by $\eta_{h}\left(x \sigma_{-l}^{h}\left(y^{*}\right)\right)$. This, in particular, implies that the set of all $L^{2}$-bounded elements is dense in $M_{*}$. It is also seen by the same reason that the set $\left\{\hat{\eta}(\phi): \phi \in M_{*}\right.$ is $L^{2}$-bounded $\}$ is dense in $\mathbf{S}$.

Now, the following lemma is easily obtained.

Lemma 2.2. (i) The functional $\omega_{x, y}$ for $x, y \in \mathfrak{a}_{0}$ is entire analytic with respect to four transformations in the above.

(ii) If $z \in \mathfrak{a}_{0}$, then the vector $\eta_{h}(z)$ belongs to the domain of the operator $J \rho^{-t} J$ and $J \rho^{-t} J \eta_{h}(z)=\eta_{h}\left(L_{i t} z\right)$ for $t \in \mathbf{R}$.

(iii) If $z \in \mathfrak{a}_{0}$, then $R\left(L_{t}\left(z^{*}\right)\right)=\left(L_{t}(R(z))\right)^{*}$ for $t \in \mathbf{R}$.

(iv) If $x^{\prime}=L_{\imath / 2}\left(R\left(\tau_{\imath / 2}\left(x^{*}\right)\right)\right)$ for $x \in \mathfrak{a}_{0}$ and $y^{\prime}=L_{\imath / 2}\left(R\left(\tau_{-l / 2}\left(y^{*}\right)\right)\right)$ for $y \in \mathfrak{a}_{0}$, then $x^{\prime}, y^{\prime} \in \mathfrak{a}_{0}$ and $\omega_{\lambda, y}^{\#}=\omega_{x^{\prime}, y^{\prime}}$.

(v) In addition, if $x^{\prime \prime}=L_{t}\left(R\left(\tau_{t / 2}\left(x^{*}\right)\right)\right)$ and $y^{\prime \prime}=R\left(\tau_{-t / 2}\left(y^{*}\right)\right)$, then $x^{\prime \prime}, y^{\prime \prime} \in \mathfrak{a}_{0}$ and $\omega_{x^{\prime}, y^{\prime}}=\omega_{x^{\prime \prime}, y^{\prime \prime}}$.

Definition 2.3. Let $\mathfrak{b}$ be the involutive subalgebra of $\left(M_{*}\right)_{\tau}$ generated by the set $\left\{\omega_{x, y}: x, y \in \mathfrak{a}_{0}\right\}$. Let $\hat{\mathfrak{d}}=\hat{\eta}(\mathfrak{b})$ be the involutive algebra with respect to the product $\hat{\eta}(\phi) \hat{\eta}(\psi)=\hat{\eta}(\phi * \psi)$ and the involution $\hat{\eta}(\phi)^{\#}=\hat{\eta}\left(\phi^{\#}\right)$. 
The purpose of this section is to show that $\hat{\mathfrak{U}}$ is a left Hilbert algebra. Let $\mathfrak{\mathfrak { A }}_{0}=\eta_{h}\left(\mathfrak{a}_{0}\right)$. Then it is a left Hilbert algebra dense in $\mathfrak{S}$ with respect to the usual operation. Hence the algebra $\mathfrak{b}$ is dense in the predual space $M_{*}$. This fact will be used in the proof of Theorem 2.17. We also use the fact that the predual $M_{*}$ is an $M$ bimodule. This bimodule structure is given by $(\phi x)(y)=\phi(x y), \quad(x \phi)(y)=\phi(y x)$ for $\phi \in M_{*}$ and $x, y \in M$. The set of $L^{2}$-bounded elements in $M_{*}$ is a left $M$-module by $x \hat{\eta}(\psi)=\hat{\eta}(x \psi)$ for $x \in M$.

Throughout the whole discussion of this paper, the mapping $\Phi: M \otimes M \rightarrow M \otimes M$ defined by

$$
\Phi(x \otimes y)=\delta(y)(x \otimes 1)
$$

plays an important role.

Lemma 2.4. (i) If $x, y \in \mathfrak{n}_{h}$, then $\Phi(x \otimes y) \in \mathfrak{n}_{h \otimes h}$. The operator $W$ on $\mathfrak{S} \otimes \mathfrak{S}$ defined by

$$
\left.W \eta_{h \otimes h}(x \otimes y)=\eta_{h \otimes h} \Phi(x \otimes y)\right), \quad x, y \in \mathfrak{n}_{h},
$$

gives an isometry $W$ on $\mathfrak{S} \otimes \mathfrak{S}$, where $\eta_{h \otimes h}$ in (2.2) is the GNS-mapping for the weight $h \otimes h$ on $M \otimes M$.

(ii) The operator $W$ defined by (2.2) satisfies $\delta(z) W=W(1 \otimes z)$ for $z \in M$.

(iii) The operator $W$ satisfies the pentagonal relation:

$$
W_{12} W_{23}=W_{23} W_{13} W_{12},
$$

where $W_{i j}$ is the tensor product of the operator $W$ acting on the $i$-th and the $j$-th spaces and the identity operator on the remaining space of $\mathbf{S} \otimes \mathfrak{S} \otimes \mathfrak{5}$.

Proof. For $x, y \in \mathfrak{n}_{h}$, since $(\mathrm{id} \otimes h)\left(\delta\left(y^{*} y\right)\right)=h\left(y^{*} y\right) 1$ by the left invariance of the Haar weight $h$, we have

$$
\begin{gathered}
(h \otimes h)\left(\Phi(x \otimes y)^{*} \Phi(x \otimes y)\right)=(h \otimes h)\left(\left(x^{*} \otimes 1\right) \delta\left(y^{*} y\right)(x \otimes 1)\right) \\
=h\left(x^{*} x\right) h\left(y^{*} y\right)=\left\|\eta_{h}(x)\right\|^{2}\left\|\eta_{h}(y)\right\|^{2} .
\end{gathered}
$$

This proves assertion (i). Assertion (ii) is immediate by replacing y by zy in (2.2). Assertion (iii) is a consequence of the coassociativity of the comultiplication $\delta$. QED

Definition 2.5. The operator $W: \mathbf{S} \otimes \mathfrak{S} \rightarrow \mathfrak{S} \otimes \mathfrak{S}$ defined by (2.2) is called the Kac-Takesaki operator.

One of the main problems in our argument is to show the unitarity of the KacTakesaki operator. Using this property, we will show that $\hat{\mathfrak{A}}$ is a left Hilbert algebra in Theorem 2.17 . 
Remark 2.6. Let $\left(A, \mu_{A}, \delta_{A}, \varepsilon_{A}, \kappa_{A}\right)$ be an involutive Hopf algebra with a faithful Haar state $h$. By the GNS construction we have a representation $\left\{\pi_{h}, \mathfrak{Q}_{h}, \eta_{h}\right\}$ such that $\left(\pi_{h}(a) \eta_{h}(b) \mid \eta_{h}(c)\right)=h\left(c^{*} a b\right)$. Then $\pi_{h}\left(a^{*}\right) \subset \pi_{h}(a)^{*}$ and hence $\pi_{h}(a)$ is closable.

Now we define two operators $V$ and $W$ on $\otimes$ by

$$
\begin{aligned}
V \eta_{h \otimes h}(a \otimes b) & =\eta_{h \otimes h}\left(\Phi_{V}(a \otimes b)\right) \\
W \eta_{h \otimes h}(a \otimes b) & =\eta_{h \otimes h}\left(\Phi_{W}(a \otimes b)\right),
\end{aligned}
$$

where $\Phi_{V}$ and $\Phi_{W}$ are the mappings of $A \otimes A$ into itself:

$$
\begin{aligned}
& \Phi_{V}=\left(\kappa_{A}^{-1} \otimes \mathrm{id}\right) \circ\left(\mu_{A} \otimes \mathrm{id}\right) \circ\left(\kappa_{A} \otimes \mathrm{id} \otimes \mathrm{id}\right) \circ\left(\mathrm{id} \otimes \delta_{A}\right) \\
& \Phi_{W}=\left(\kappa_{A}^{-1} \otimes \mathrm{id}\right) \circ\left(\mu_{A} \otimes \mathrm{id}\right) \circ\left(\kappa_{A} \otimes \kappa_{A} \otimes \mathrm{id}\right) \circ\left(\mathrm{id} \otimes \delta_{A}\right) .
\end{aligned}
$$

Due to the existence of the counit, it is shown that $\Phi_{W}=\Phi$ and $V W=W V=1$, where $\Phi$ is defined by the same formula as (2.1). The unitarity of the Kac-Takesaki operator for this case is then an immediate consequence deduced from the existence of a faithful Haar state on an involutive Hopf algebra contrary to the case of Woronowicz algebras as discussed below.

Lemma 2.7. The isometric operator $W$ acting on the Hilbert space $\otimes$ belongs to the von Neumann algebra $M \otimes \mathscr{L}(\mathfrak{S})$.

Proof. For $x_{j}, y_{j} \in \mathfrak{a}_{h}, j=1,2$,

$$
\begin{aligned}
(J \otimes J)\left(y_{1} \otimes y_{2}\right)(J \otimes J) W \eta_{h \otimes h}\left(x_{1} \otimes x_{2}\right) & \\
= & (J \otimes J)\left(y_{1} \otimes y_{2}\right)(J \otimes J) \eta_{h \otimes h}\left(\delta\left(x_{2}\right)\left(x_{1} \otimes 1\right)\right) \\
& =\delta\left(x_{2}\right)\left(x_{1} \otimes 1\right)(J \otimes J) \eta_{h \otimes h}\left(y_{1} \otimes y_{2}\right) \\
& =\delta\left(x_{2}\right)\left(J y_{1} J \otimes 1\right)\left(\eta_{h}\left(x_{1}\right) \otimes J \eta_{h}\left(y_{2}\right)\right),
\end{aligned}
$$

where we used the fact that $J y J \eta_{h}(x)=x J \eta_{h}(y), x, y \in \mathfrak{a}_{h}$ for the second and the third equalities. Therefore, the existence of the strong net $y_{1} \rightarrow 1$ implies

$$
\left(1 \otimes J y_{2} J\right) W \eta_{h \otimes h}\left(x_{1} \otimes x_{2}\right)=\delta\left(x_{2}\right)\left(\eta_{h}\left(x_{1}\right) \otimes J \eta_{h}\left(y_{2}\right)\right) .
$$

Due to the density of $\mathfrak{a}_{h}=\eta_{h}\left(\mathfrak{a}_{h}\right)$ in $\mathfrak{S}, \eta_{h}\left(x_{1}\right)$ can be replaced by $z^{\prime} \xi$ for $z^{\prime} \in M^{\prime}$ and $\xi \in \mathfrak{S}$ and hence we obtain

$$
\begin{aligned}
& \left(1 \otimes J y_{2} J\right) W\left(z^{\prime} \xi \otimes \eta_{h}\left(x_{2}\right)\right)=\delta\left(x_{2}\right)\left(z^{\prime} \xi \otimes J \eta_{h}\left(y_{2}\right)\right) \\
& \quad=\left(z^{\prime} \otimes 1\right) \delta\left(x_{2}\right)\left(\xi \otimes J \eta_{h}\left(y_{2}\right)\right)=\left(z^{\prime} \otimes J y_{2} J\right) W\left(\xi \otimes \eta_{h}\left(x_{h}\right)\right) .
\end{aligned}
$$

Therefore the existence of the strong net $y_{2} \rightarrow 1$ and the density of $\eta_{h}\left(\mathfrak{a}_{h}\right)$ in implies $W\left(z^{\prime} \otimes 1\right)=\left(z^{\prime} \otimes 1\right) W$ for $z^{\prime} \in M^{\prime}$ and proves the assertion.

Lemma 2.8. Let $\varphi, \psi$ be the elements in $M_{*}$. If $\psi$ is $L^{2}$-bounded, then the product $\phi * \psi$ is $L^{2}$-bounded with its $L^{2}$-norm satisfying $\|\hat{\eta}(\phi * \psi)\| \leq\|\phi\|\|\hat{\eta}(\psi)\|$. 
Proof. Let $\varphi, \psi \in M_{*}$ be $L^{2}$-bounded elements and $x, y \in \mathfrak{a}_{h}$. We define a linear functional $\omega_{\psi, x}$ on $\mathscr{L}(\mathfrak{S})$ by $\omega_{\psi, x}(z)=\left(z \hat{\eta}(\psi) \mid \eta_{h}(x)\right), z \in \mathscr{L}(\mathfrak{Q})$. It is easy to see that $\varphi y^{*}=\omega_{\varphi, y}$. Then by using $W \in M \otimes \mathscr{L}(\mathfrak{S})$ which was proved in Lemma 2.7, we have

$$
\begin{aligned}
\left(\varphi y^{*} \otimes \omega_{\psi, x}\right)\left(W^{*}\right) & =\left(W^{*} \hat{\eta}(\varphi) \otimes \hat{\eta}(\psi) \mid \eta_{h}(y) \otimes \eta_{h}(x)\right) \\
& =\left(\hat{\eta}(\varphi) \otimes \hat{\eta}(\psi) \mid \eta_{h \otimes h}(\delta(x)(y \otimes 1))\right. \\
& =(\varphi \otimes \psi)\left(\left(y^{*} \otimes 1\right) \delta\left(x^{*}\right)\right)=\left(\varphi y^{*} \otimes \psi\right)\left(\delta\left(x^{*}\right)\right) .
\end{aligned}
$$

The existence of the strong net $y^{*} \rightarrow 1$ combined with the density of $L^{2}$-bounded elements in the predual $M_{*}, \varphi y^{*}$ in the both sides of the above equality can be replaced by an 'arbitrary element $\phi \in M_{*}$ Therefore $\left|(\phi \otimes \psi)\left(\delta\left(x^{*}\right)\right)\right|=\left|\left(\phi \otimes \omega_{\psi, \lambda}\right)\left(W^{*}\right)\right| \leq$ $\|\phi\|\|\hat{\eta}(\psi)\|\left\|\eta_{h}(x)\right\|$. This proves the assertion.

QED

Definition 2.9. We denote by $\hat{\pi}(\phi)$ for $\phi \in M_{*}$ the element in $\mathscr{L}(\mathfrak{S})$ determined by $\hat{\pi}(\phi) \hat{\eta}(\psi)=\hat{\eta}(\phi * \psi)$ for all $L^{2}$-bounded elements $\psi$ in $M_{*}$, and by $\hat{M}$ the von Neumann algebra generated by the set $\left\{\hat{\pi}(\phi): \phi \in M_{*}\right\}$

The mapping $\hat{\pi}: M_{*} \rightarrow \hat{M}$ is called the Fourier transform. By Lemma 2.8 , it is bounded : $\|\hat{\pi}(\phi)\| \leq\|\phi\|$ for $\phi \in M_{*}$.

Lemma 2.10. (i) $\hat{\pi}(\phi * \psi)=\hat{\pi}(\phi) \hat{\pi}(\psi)$ for $\phi, \psi \in M_{*}$;

(ii) $\left(\xi \otimes \xi^{\prime} \mid W\left(\eta \otimes \eta^{\prime}\right)\right)=\left(\hat{\pi}\left(\omega_{\xi, \eta}\right) \xi^{\prime} \mid \eta^{\prime}\right)$ for $\xi, \xi^{\prime}, \eta, \eta^{\prime} \in \mathfrak{g}$, where $\omega_{\xi, \eta}$ is identified with an element in $M_{*}$;

(iii) $\hat{\pi}(\phi)=(\phi \otimes \mathrm{id})\left(W^{*}\right)$ for $\phi \in M_{*}$; and

(iv) $W \in M \otimes \hat{M}$.

Proof. (i) It is clear from Definition 2.9.

(ii) Due to the proof of Lemma 2.8,

$$
\left(\hat{\eta}(\varphi) \otimes \hat{\eta}(\psi) \mid W \eta_{h \otimes h}(x \otimes y)\right)=\left(\varphi x^{*} \otimes \psi\right)\left(\delta\left(y^{*}\right)\right) .
$$

for $L^{2}$-bounded elements $\varphi, \psi$ in $M_{*}$ and $x, y \in \mathfrak{a}_{h}$. Therefore, using $\varphi x^{*}=\omega_{\varphi, 1}$, we have

$$
\left(\hat{\eta}(\varphi) \otimes \hat{\eta}(\psi) \mid W \eta_{h \otimes h}(x \otimes y)\right)=\left(\omega_{\varphi \cdot 1} * \psi\right)\left(y^{*}\right)=\left(\hat{\pi}\left(\omega_{\varphi, 1}\right) \hat{\eta}(\psi) \mid \eta_{h}\left(y^{\prime}\right)\right) .
$$

Then the density argument combined with the inequality $\left\|\hat{\pi}\left(\omega_{\xi, \eta}\right)\right\| \leq\left\|\omega_{\xi, \eta}\right\| \leq\|\xi\|\|\eta\|$, we obtain the assertion.

Assertion (iii) is clear from assertion (ii). Since $W \in M \otimes \mathscr{L}^{\prime}(\mathfrak{S})$ by Lemma 2.7, assertion (iv) follows from assertion (ii) by replacing $\xi^{\prime}$ by $y^{\prime} \xi^{\prime}$ with $y^{\prime} \in \hat{M}^{\prime}$. QED 
Lemma 2.11. For $\phi \in\left(M_{*}\right)_{\tau}, \hat{\pi}\left(\phi^{\#}\right)=\hat{\pi}(\phi)^{*}$.

Proof. Let $x_{j}, y_{j} \in \mathfrak{a}_{0}$ and put $z_{j}=x_{j} \sigma_{-i}^{h}\left(y_{j}^{*}\right)$ for $j=1,2$. Then, for $\phi \in\left(M_{*}\right)_{\tau}$,

$$
\begin{aligned}
\left(\hat{\pi}\left(\phi^{\#}\right) \hat{\eta}\left(\omega_{x_{1}, y_{1}}\right) \mid \hat{\eta}\left(\omega_{x_{2}, y_{2}}\right)\right) & =\left(\phi^{\#} \otimes \omega_{x_{1}, y_{1}}\right)\left(\delta\left(z_{2}^{*}\right)\right) \\
& =\left(\phi^{\#} \otimes h\right)\left(\left(1 \otimes y_{1}^{*}\right)\left(\delta\left(z_{2}^{*}\right)\left(1 \otimes x_{1}\right)\right) .\right.
\end{aligned}
$$

Here we use the KMS condition for the weight $h$. Then the right hand side is equal to

$$
\begin{aligned}
\left(\phi^{\#} \otimes h\right)\left(\delta\left(z_{2}^{*}\right)\left(1 \otimes z_{1}\right)\right) & =\left(\phi^{\#} \circ \tau_{i / 2} \circ R \otimes h\right)\left(\left(1 \otimes z_{2}^{*}\right) \delta\left(z_{1}\right)\right) \\
& =\left(\phi^{*} \otimes h\right)\left(\left(1 \otimes z_{2}^{*}\right) \delta\left(z_{1}\right)\right)=\overline{(\phi \otimes h)\left(\delta\left(z_{1}^{*}\right)\left(1 \otimes z_{2}\right)\right)} \\
& =\overline{\left(\phi \otimes \omega_{x_{2}, y_{2}}\right)\left(\delta\left(z_{1}^{*}\right)\right)}=\overline{\left(\hat{\pi}(\phi) \hat{\eta}\left(\omega_{x_{2}, y_{2}}\right) \mid \hat{\eta}\left(\omega_{x_{1}, y_{1}}\right)\right)} \\
& =\left(\hat{\eta}\left(\omega_{x_{1}, y_{1}}\right) \mid \hat{\pi}(\phi) \hat{\eta}\left(\omega_{x_{2}, y_{2}}\right)\right),
\end{aligned}
$$

where the first equality follows from the strong left invariance and the second equality follows from $\phi^{\#} \circ \tau_{t / 2} \circ R=\phi^{*}$. Therefore the density argument proves the assertion.

QED

By Lemmas 2.10 and 2.11 , the Fourier transform $\hat{\pi}$ is a $*$-homomorphism of $\left(M_{*}\right)_{\tau}$ to $\hat{M}$. To show the unitarity of the Kac-Takesaki operator we need the following lemma giving a relation between the Fourier transform with the left involution operator $S$, the right involution operator $F$, or the modular operator $\Delta$ for the left Hilbert algebra $\mathfrak{A}_{h}=\eta_{h}\left(\mathfrak{a}_{h}\right)$. In what follows, the domain of an unbounded operator $T$ will be denoted by $\operatorname{Dom}(T)$.

Lemma 2.12. (i) If $\phi \in M_{*}$, then $\hat{\pi}(\phi) \operatorname{Dom}(F) \subset \operatorname{Dom}(F)$ and

$$
F \hat{\pi}(\phi) \xi^{\prime}=\hat{\pi}\left(\phi^{*}\right) F \xi^{\prime} \text { for } \xi^{\prime} \in \operatorname{Dom}(F) .
$$

(ii) If $\phi \in\left(M_{*}\right)_{\tau}$, then $\hat{\pi}(\phi) \operatorname{Dom}(S) \subset \operatorname{Dom}(S)$ and

$$
S \hat{\pi}(\phi) \xi=\hat{\pi}\left(\phi^{*} \circ \tau_{-\imath}\right) S \xi \text { for } \xi \in \operatorname{Dom}(S) .
$$

(iii) If $\phi \in\left(M_{*}\right)_{\tau}$, then $\hat{\pi}(\phi) \operatorname{Dom}(\Delta) \subset \operatorname{Dom}(\Delta)$ and

$$
\Delta \hat{\pi}(\phi) \xi=\hat{\pi}\left(\phi \circ \tau_{1}\right) \Delta \xi \quad \text { for } \xi \in \operatorname{Dom}(\Delta) .
$$

Proof. (i) Let $\mathfrak{a}^{\prime}$ be the right Hilbert algebra corresponding to the left Hilbert algebra $\mathfrak{A}_{h}$. If $\psi=\omega_{\xi^{\prime}, \eta^{\prime}}$ for $\xi^{\prime}, \eta^{\prime} \in \mathfrak{A}^{\prime}$, then $\hat{\eta}(\psi)=\xi^{\prime} \eta^{\prime b}$ where the superscript $b$ indicates the right involution in $\mathfrak{A}^{\prime}$. Hence $\psi$ and $\psi^{*}$ are $L^{2}$-bounded. For any $\phi \in M_{*}$ and $x \in \mathfrak{a}_{h}$, we see that

$$
\begin{gathered}
\left(\hat{\pi}(\phi) \hat{\eta}(\psi) \mid S \eta_{h}(x)\right)=\left(\hat{\eta}(\phi * \psi) \mid \eta_{h}\left(x^{*}\right)\right)=(\phi * \psi)(x) \\
=(\phi \otimes \psi)(\delta(x))=\overline{\left(\phi^{*} \otimes \psi^{*}\right)\left(\delta\left(x^{*}\right)\right)}=\left(\eta_{h}(x) \mid \hat{\pi}\left(\phi^{*}\right) \hat{\eta}\left(\psi^{*}\right)\right) .
\end{gathered}
$$


Therefore $\hat{\pi}(\phi) \hat{\eta}(\psi)$ belongs to the domain of the right involution operator $F$ and $F \hat{\pi}(\phi) \hat{\eta}(\psi)=\hat{\pi}\left(\phi^{*}\right) \hat{\eta}\left(\psi^{*}\right)$. Since

$$
\left(F \hat{\eta}(\psi) \mid \eta_{h}(x)\right)=\left(\eta_{h}\left(x^{*}\right) \mid \hat{\eta}(\psi)\right)=\overline{\psi(x)}=\psi^{*}\left(x^{*}\right)=\left(\hat{\eta}\left(\psi^{*}\right) \mid \eta_{h}(x)\right)
$$

for any $x \in \mathfrak{a}_{h}$, we have a formula

$$
F \hat{\eta}(\psi)=\hat{\eta}\left(\psi^{*}\right)
$$

Hence $F \hat{\pi}(\phi) \hat{\eta}(\psi)=\hat{\pi}\left(\phi^{*}\right) F \hat{\eta}(\psi)$.

Let $\xi^{\prime}$ be an arbitrary element in the domain of the operator $F$. Since the linear span $\left(\mathfrak{U}^{\prime}\right)^{2}$ of elements of the form $\xi^{\prime} \eta^{\prime}$ with $\xi^{\prime}, \eta^{\prime} \in \mathfrak{A}^{\prime}$ is a core for $F$, there exists a sequence $\left\{\zeta_{n}^{\prime}\right\}$ in $\left(\mathfrak{A}^{\prime}\right)^{2}$ such that $\zeta_{n}^{\prime} \rightarrow \xi^{\prime}$ and $F \zeta_{n}^{\prime} \rightarrow F \xi^{\prime}$. Since $\hat{\eta}\left(\omega_{\xi^{\prime}, \eta^{\prime}}\right)=\xi^{\prime} \eta^{\prime b}$ for $\xi^{\prime}, \eta^{\prime} \in \mathfrak{A}^{\prime}$, it follows that $\hat{\pi}(\phi) \zeta_{n}^{\prime} \rightarrow \hat{\pi}(\phi) \xi^{\prime}$ and $F \hat{\pi}(\phi) \zeta_{n}^{\prime}=\hat{\pi}\left(\phi^{*}\right) F \zeta_{n}^{\prime}$ $\rightarrow \hat{\pi}\left(\phi^{*}\right) F \xi^{\prime}$. Consequently, $\hat{\pi}(\phi) \xi^{\prime}$ belongs to the domain of $F$ and $F \hat{\pi}(\phi) \xi^{\prime}=$ $\hat{\pi}\left(\phi^{*}\right) F \xi^{\prime}$.

(ii) If $\xi \in \operatorname{Dom}(S)$ and $\xi^{\prime} \in \operatorname{Dom}(F)$, then

$$
\left(F \xi^{\prime} \mid \hat{\pi}(\phi) \xi\right)=\left(\hat{\pi}\left(\phi^{\#}\right) F \xi^{\prime} \mid \xi\right)=\left(F \hat{\pi}\left(\phi^{\# *}\right) \xi^{\prime} \mid \xi\right)=\left(S \xi \mid \hat{\pi}\left(\phi^{\# *}\right) \xi^{\prime}\right)=\left(\hat{\pi}\left(\phi^{\# \# \#}\right) S \xi \mid \xi^{\prime}\right) .
$$

Since $\phi^{\# * \#}=\phi^{*} \circ \tau_{-i}$, it follows that $\hat{\pi}(\phi) \xi$ belongs to the domain of the operator $S$ and $S \hat{\pi}(\phi) \xi=\hat{\pi}\left(\phi^{*} \circ \tau_{-l}\right) S \xi$.

(iii) If $\xi \in \operatorname{Dom}(\Delta)$, then $S \xi \in \operatorname{Dom}(F)$ and hence

$$
\hat{\pi}\left(\phi \circ \tau_{\imath}\right) F S \xi=F \hat{\pi}\left(\phi^{*} \circ \tau_{-l}\right) S \xi=F S \hat{\pi}(\phi) \xi
$$

by (i) and (ii).

QED

Lemma 2.13. Let $\hat{\mathfrak{U}}=\hat{\eta}(\mathfrak{b})$. Then the followings hold:

(i) As a linear space, $\hat{\mathfrak{U}}$ coincides with $\mathfrak{Z}_{0}^{2}$, and hence $\hat{\mathfrak{U}}$ is dense in $\mathfrak{\mathfrak { S }}$.

(ii) The left involution operator: $\hat{\eta}(\phi) \rightarrow \hat{\eta}\left(\phi^{\#}\right)$ in $\hat{\mathfrak{U}}$ is closable as a conjugate linear operator on $\mathbf{s}$.

Proof. Assertion (i) is a consequence of $\hat{\eta}\left(\omega_{\imath, v}\right)=\eta_{h}\left(x \sigma_{-l}^{h}\left(y^{*}\right)\right)$ for $x, y \in \mathfrak{a}_{0}$. We have only to show assertion (ii). If $\hat{\eta}\left(\phi_{n}\right) \rightarrow 0$ and $\hat{\eta}\left(\phi_{n}^{\#}\right) \rightarrow \xi$ for $\phi_{n} \in\left(M_{*}\right)_{\tau}$, then, for each $\eta_{h}(x) \in \mathfrak{A}_{0}=\eta_{h}\left(\mathfrak{a}_{0}\right)$,

$$
\phi_{n}^{\#}\left(x^{*}\right)=\left(\hat{\eta}\left(\phi_{n}^{\#}\right) \mid \eta_{h}(x)\right) \rightarrow\left(\xi \mid \eta_{h}(x)\right) .
$$

Since $\phi_{n}^{\#}\left(x^{*}\right)=\left(\eta_{h}\left(\tau_{-t / 2} \circ R\left(x^{*}\right)\right) \mid \hat{\eta}\left(\phi_{n}\right)\right)$ converges to $0,(\xi \mid \eta)=0$ for all $\eta \in \mathfrak{A}_{0}$. Thus $\xi=0$.

Let $\hat{S}$ denote the closed operator on $\mathfrak{S}$ obtained as the closure of the conjugate linear mapping: $\hat{\eta}(\phi) \rightarrow \hat{\eta}\left(\phi^{\#}\right)$ and $\hat{S}=\hat{J} \hat{\Delta}^{1 / 2}$ be the polar decomposition. Since $\hat{\mathfrak{U}}$ is a core for $\hat{S}$ and $\hat{S}^{2}=1$ on it, the range of $\hat{S}$ is then contained in the domain of $\hat{S}$ and 
$\hat{S}^{2} \subset 1$. Indeed, if $\eta=\hat{S} \xi$ for $\xi \in \operatorname{Dom}(\hat{S})$, there exists a sequence $\left\{\xi_{n}\right\}$ in $\hat{\mathfrak{U}}$ such that $\xi_{n} \rightarrow \xi$ and $\hat{S} \xi_{n} \rightarrow \eta$. Since $\hat{S}\left(\hat{S} \xi_{n}\right)=\xi_{n} \rightarrow \xi$ and $\hat{S}$ is closable, $\eta \in \operatorname{Dom}(\hat{S})$ and $\hat{S} \eta=\xi$. Therefore $\hat{\Delta}^{1 / 2} \hat{J} \hat{\Delta}^{1 / 2}=\hat{J}$ on the domain of $\hat{\Delta}^{1 / 2}$. Hence $\hat{J} \operatorname{Dom}\left(\hat{\Delta}^{1 / 2}\right)$ $\subset \operatorname{Dom}\left(\hat{\Delta}^{-1 / 2}\right)$ and

$$
\hat{\Delta}^{1 / 2}=\hat{J} \hat{\Delta}^{-1 / 2} \hat{J} \quad \text { on } \operatorname{Dom}\left(\hat{\Delta}^{1 / 2}\right) \text {. }
$$

Let $\hat{F}$ be the adjoint operator of $\hat{S}$. Then $\hat{F}=\hat{\Delta}^{1 / 2} \hat{J}$. If $\phi \in \mathfrak{b}$ and $x \in \mathfrak{a}_{0}$, then

$$
\left(\hat{S} \hat{\eta}(\phi) \mid \eta_{h}(x)\right)=\phi^{*}\left(\tau_{-l / 2} \circ R\left(x^{*}\right)\right)=\overline{\phi\left(\tau_{-l / 2} \circ R\left(x^{*}\right)^{*}\right)}=\left(\eta_{h}\left(\tau_{-l / 2} \circ R\left(x^{*}\right)\right) \mid \hat{\eta}(\phi)\right) .
$$

Hence the set $\mathfrak{U}_{0}$ is contained in the domain of the operator $\hat{F}$ and

$$
\hat{F} \eta_{h}(x)=\eta_{h}\left(\tau_{-t / 2} \circ R\left(x^{*}\right)\right) \text { for } x \in \mathfrak{a}_{0} .
$$

The $\left\{\tau_{t}\right\}$-invariance of the Haar weight $h$ implies $\tau_{t}\left(\mathfrak{n}_{h}\right)=\mathfrak{n}_{h}$ and the existence of a positive self-adjoint operator $H$ with

$$
H^{t t} \eta_{h}(x)=\eta_{h}\left(\tau_{t}(x)\right), \quad t \in \mathbb{R}, \quad x \in \mathfrak{n}_{h} .
$$

Hence $S H^{t t} \eta_{h}(x)=\eta_{h}\left(\tau_{t}(x)^{*}\right)=\eta_{h}\left(\tau_{t}\left(x^{*}\right)\right)=H^{t t} S \eta_{h}(x)$ for $x \in \mathfrak{a}_{h}$, where $S$ is the left involution operator for the left Hilbert algebra $\mathfrak{2}_{h}=\eta_{h}\left(\mathfrak{a}_{h}\right)$. Since the set $\mathfrak{A}_{h}$ is a core for $\Delta^{1 / 2}$ and satisfies $H^{t t} \eta_{h}\left(\mathfrak{n}_{h}\right)=\eta_{h}\left(\tau_{t}\left(\mathfrak{n}_{h}\right)\right)=\eta_{h}\left(\mathfrak{n}_{h}\right)$, we have $S H^{t}=H^{t t} S$ for any $t \in \mathbb{R}$. As the one parameter automorphism groups $\left\{\sigma_{t}^{h}\right\},\left\{\sigma_{t}^{h \circ R}\right\}$ and $\left\{\tau_{t}\right\}$ are mutually commutative and preserve the Haar weight $h$, the one parameter unitaries $\left\{\Delta^{t t}\right\},\left\{\Delta_{h \circ R}^{t t}\right\}$ and $\left\{H^{t}\right\}$ which implement the above automorphism groups are mutually commutative. This implies $J H^{t t}=H^{t t} J$ and hence $J H J=H^{-1}$.

The family $\{J, \Delta, \rho, H\}$ in the following lemma will be crucial for the analysis of Woronowicz algebras.

Lemma 2.14. (i) The four positive self-adjoint operators $\Delta, H, \rho$ and $J \rho J$ are mutually commutative.

(ii) $J H J=H^{-1}$

(iii) The operator $\hat{\Delta}$ is the closure of the operator $J \rho J H$.

Proof. Assertions (i) and (ii) have already been discussed above except for the commutativity of $\rho$ and $J \rho J$ with $\Delta$ and $H$. Since $\rho$ is affiliated with the fixed point subalgebra of $M$ with respect to the modular automorphism $\left\{\sigma_{t}^{h}\right\}, \Delta$ commutes with $\rho$ and $J \rho J$. Due to the $\left\{\tau_{t}\right\}$-invariance of the Haar weight, we have

$$
\tau_{t}\left(\rho^{-l s}\right)=\tau_{t}((D h \circ R: D h),)=\left(D h \circ R \circ \tau_{t}^{-1}: D h \circ \tau_{t}^{-1}\right),=(D h \circ R: D h),=\rho^{-r s} .
$$

Thus $H^{\prime \prime}$ commutes with $\rho^{\prime \prime}$. Hence $H$ commutes with $\rho$ as well as $J \rho J$ by (ii).

(iii) The left Hilbert algebra $\mathfrak{U}_{0}$ is contained in the domain of $\hat{F}$ by (2.6). For any $x$ and $y$ in $a_{0}$, we have

$$
\left(\hat{\Delta}^{-1 / 2} \eta_{h}(x) \mid \hat{\Delta}^{-1 / 2} \eta_{h}(y)\right)=\left(\hat{F} \eta_{h}(y) \mid \hat{F} \eta_{h}(x)\right)
$$




$$
\begin{aligned}
& =\left(\eta_{h}\left(R \circ \tau_{-l / 2}\left(y^{*}\right)\right) \mid \eta_{h}\left(R \circ \tau_{-l / 2}\left(x^{*}\right)\right)\right) \\
& =h \circ R\left(\tau_{-l / 2}\left(y^{*}\right) \tau_{-l / 2}\left(x^{*}\right)^{*}\right) \\
& =\left(J \rho^{-1 / 2} J \eta_{h}\left(\tau_{l / 2}(x)\right) \mid J \rho^{-1 / 2} J \eta_{h}\left(\tau_{l / 2}(y)\right)\right) \\
& =\left(J \rho^{-1 / 2} J H^{-1 / 2} \eta_{h}(x) \mid J \rho^{-1 / 2} J H^{-1 / 2} \eta_{h}(y)\right),
\end{aligned}
$$

where the fourth equality is due to Lemma 2.2. Since $\mathfrak{A}_{0}=\eta_{h}\left(\mathfrak{a}_{0}\right)$ is a core for $H^{-1 / 2}$ and for $J \rho^{-1 / 2} J H^{-1 / 2}$, it follows that $J \rho^{-1 / 2} J H^{-1 / 2} \subset \hat{\Delta}^{-1 / 2}$. The operators in the both sides are self-adjoint. Hence the closure of $J \rho^{-1 / 2} J H^{-1 / 2}$ coincides with $\hat{\Delta}^{-1 / 2}$. Thus the commutativity of $J \rho J$ and $H$ implies that the closure of $J \rho J H$ is nothing but the operator $\hat{\Delta}$.

QED

Corollary 2.14.1. The deformation automorphism $\left\{\tau_{t}\right\}$ on $M$ is implemented by $\left\{\hat{\Delta}^{t t}\right\}$ as well as $\left\{H^{t t}\right\}$. Namely, the equalities $\tau_{t}(x)=\hat{\Delta}^{t t} x \hat{\Delta}^{-t t}=H^{t t} x H^{-t t}$ hold for all $x \in M$ and $t \in \mathbf{R}$.

Lemma 2.15. (i) The left Hilbert algebra $\mathfrak{\mathfrak { A }}_{0}$ is a core for both involution operators $\hat{S}$ and $\hat{F}$.

(ii) $\hat{S} \eta_{h}(x)=\eta_{h}\left(L_{t} \circ R \circ \tau_{t / 2}\left(x^{*}\right)\right)$ for $x \in \mathfrak{a}_{0}$.

(iii) $\hat{F} \hat{\eta}(\phi)=\hat{\eta}\left(\left(\rho^{-1} \phi^{*}\right) \circ R \circ \tau_{t / 2}\right)$ for $\phi \in \mathfrak{b}$.

Proof. (i) Let $x \in \mathfrak{a}_{0}$. Then, by Lemma 2.14 (iii),

$$
\begin{aligned}
\hat{\Delta}^{t t} \eta_{h}(x) & =J \rho^{-t t} J H^{t t} \eta_{h}(x)=J \rho^{-t t} J \eta_{h}\left(\tau_{t}(x)\right)=S \rho^{-t t} S \eta_{h}\left(\tau_{t}(x)\right) \\
& =S \eta_{h}\left(\rho^{-t t} \tau_{t}(x)^{*}\right)=\eta_{h}\left(\tau_{t}(x) \rho^{t t}\right)=\eta_{h}\left(L_{t}\left(\tau_{t}(x)\right)\right) .
\end{aligned}
$$

Hence the vector $\eta_{h}(x)$ is entire analytic for $\left\{\hat{\Delta}^{t t}\right\}$ and $\mathfrak{A}_{0}$ is a core for the operators $\hat{\Delta}^{1 / 2}$ and $\hat{\Delta}^{-1 / 2}$. Thus the assertion is proved.

(ii) It is immediate from (2.6) that, for $x, y \in \mathfrak{a}_{0}$

$$
\begin{aligned}
\left(\hat{S} \eta_{h}(x) \mid \eta_{h}(y)\right) & =\left(\eta_{h}\left(R \circ \tau_{-t / 2}\left(y^{*}\right)\right) \mid \eta_{h}(x)\right)=h\left(x^{*} R \circ \tau_{-t / 2}\left(y^{*}\right)\right) \\
& =h \circ R\left(y^{*} R \circ \tau_{t / 2}\left(x^{*}\right)\right)=\left(\eta_{h}\left(L_{\iota} \circ R \circ \tau_{t / 2}\left(x^{*}\right)\right) \mid \eta_{h}(y)\right),
\end{aligned}
$$

where the fourth equality is due to Lemma 2.2 .

(iii) It is immediate from (ii).

Theorem 2.16. Let $W$ be the Kac-Takesaki operator. Then

(i) $W$ is a unitary in $M \otimes \hat{M}$;

(ii) W commutes with $\hat{\Delta} \otimes \Delta$; and

(iii) $W^{*}=(\hat{J} \otimes J) W(\hat{J} \otimes J)$. 
Proof. Assertions (i) and (ii) will be proved simultaneously. Let $\mathfrak{a}^{\prime}$ be the right Hilbert algebra associated with $\mathfrak{A}_{h}$. Then, for $x, y \in \mathfrak{a}_{0}, \xi^{\prime} \in \mathfrak{A}^{\prime}$ and $\eta \in \mathfrak{A}_{h}$, $\hat{\pi}\left(\omega_{x, v}\right) \xi^{\prime} \in \operatorname{Dom}(F)$ and $F \hat{\pi}\left(\omega_{x, y}\right) \xi^{\prime}=\hat{\pi}\left(\omega_{y, x}\right) F \xi^{\prime}$ by Lemma 2.12 (i). Hence, by Lemmas 2.2 and 2.10 (ii), we have

$$
\begin{aligned}
\left(\eta_{h}(x) \otimes \xi^{\prime} \mid W\left(\eta_{h}(y) \otimes \eta\right)\right) & =\left(\hat{\pi}\left(\omega_{x, y}\right) \xi^{\prime} \mid \eta\right)=\left(S \eta \mid F \hat{\pi}\left(\omega_{x, y}\right) \xi^{\prime}\right) \\
& =\left(S \eta \mid \hat{\pi}\left(\omega_{y, x}\right) F \xi^{\prime}\right) .
\end{aligned}
$$

Hence, by Lemma 2.11, the right hand side is equal to

$$
\begin{aligned}
\left(\hat{\pi}\left(\left(\omega_{y, x}\right)^{\#}\right) S \eta \mid F \xi^{\prime}\right) & =\left(\hat{\pi}\left(\omega_{L_{t} \circ R \circ \tau_{t / 2}\left(y^{*}\right), R \circ \tau_{-l / 2}\left(x^{*}\right)}\right) S \eta \mid F \xi^{\prime}\right) \\
& =\left(\eta_{h}\left(L_{\iota} \circ R \circ \tau_{\iota / 2}\left(y^{*}\right)\right) \otimes S \eta \mid W\left(\eta_{h}\left(R \circ \tau_{-l / 2}\left(x^{*}\right)\right) \otimes F \xi^{\prime}\right)\right) \\
& =\left(\hat{S} \eta_{h}(y) \otimes S \eta \mid W\left(\hat{F} \eta_{h}(x) \otimes F \xi^{\prime}\right)\right),
\end{aligned}
$$

where the first equality follows from Lemma 2.2 and the third from (2.6) and Lemma 2.15. Let $\hat{S} \otimes S, \hat{F} \otimes F$ and $\hat{\Delta} \otimes \Delta$ denote the closures of their algebraic tensor products. Then $\hat{\Delta} \otimes \Delta$ is positive self-adjoint and $\hat{F} \otimes F=(\hat{S} \otimes S)^{*}=(\hat{\Delta} \otimes \Delta)^{1 / 2}(\hat{J} \otimes J)$. By virtue of Lemma $2.15, \mathfrak{A}_{0} \otimes \mathfrak{A}_{0}$ is a core for both operators $\hat{S} \otimes S$ and $\hat{F} \otimes F$. Thus

(2.8) $(\tilde{\xi} \mid W \tilde{\eta})=((\hat{S} \otimes S) \tilde{\eta} \mid W(\hat{F} \otimes F) \tilde{\xi})$ for $\tilde{\xi} \in \operatorname{Dom}(\hat{F} \otimes F)$ and $\tilde{\eta} \in \operatorname{Dom}(\hat{S} \otimes S)$.

Replacing $\xi^{\prime} \in \mathfrak{A}^{\prime}$ and $\eta \in \mathfrak{A}_{h}$ in the above argument by $\xi \in \mathfrak{A}_{h}$ and $\eta^{\prime} \in \mathfrak{A}^{\prime}$, respectively, we obtain a similar equation

(2.9) $(\tilde{\xi} \mid W \tilde{\eta})=((\hat{F} \otimes F) \tilde{\eta} \mid W(\hat{S} \otimes S) \tilde{\xi})$ for $\tilde{\eta} \in \operatorname{Dom}(\hat{F} \otimes F)$ and $\tilde{\xi} \in \operatorname{Dom}(\hat{S} \otimes S)$.

Next we will show that

$$
W(\hat{F} \otimes F)=(\hat{F} \otimes F) W^{*} \text { and } W^{*}(\hat{F} \otimes F)=(\hat{F} \otimes F) W \text { on } \operatorname{Dom}(\hat{F} \otimes F)
$$

and

$$
W(\hat{S} \otimes S)=(\hat{S} \otimes S) W^{*} \text { on } \operatorname{Dom}(\hat{S} \otimes S) .
$$

By virtue of (2.8), the vector $W(\hat{F} \otimes F) \hat{\xi}$ belongs to the domain of $\hat{F} \otimes F$ and

$$
W^{*}=(\hat{F} \otimes F) W(\hat{F} \otimes F) \text { on } \operatorname{Dom}(\hat{F} \otimes F) .
$$

Hence $W^{*} \operatorname{Dom}(\hat{F} \otimes F)$ is contained in the range of $\hat{F} \otimes F$. Since $\hat{F} \otimes F$ is closed and involutive on the core $\mathfrak{A}_{0} \otimes \mathfrak{A}_{0}$, the range is contained in its domain and $(\hat{F} \otimes F)^{2}=1$ on $\operatorname{Dom}(\hat{F} \otimes F)$. Thus the first equality in (2.10) follows from (2.12). We also deduce from (2.9) that the second equality in (2.10) holds. By considering the adjoint of the first equality in (2.10), we obtain (2.11).

Finally we will show that $W$ is unitary. Since $(\hat{S} \otimes S) \operatorname{Dom}(\hat{\Delta} \otimes \Delta)$ $\subset \operatorname{Dom}(\hat{F} \otimes F)$, we have $\hat{\Delta} \otimes \Delta \subset(\hat{F} \otimes F) W^{*} W(\hat{S} \otimes S)$. The equality $W(\hat{S} \otimes S)$ $=(\hat{S} \otimes S) W^{*}$ holds on the domain of the operator $\hat{\Delta} \otimes \Delta$ by (2.11). Hence 


$$
\hat{\Delta} \otimes \Delta \subset(\hat{F} \otimes F) W^{*}(\hat{S} \otimes S) W^{*}
$$

and

$$
(\hat{S} \otimes S) W^{*} \operatorname{Dom}(\hat{\Delta} \otimes \Delta)=W(\hat{S} \otimes S) \operatorname{Dom}(\hat{\Delta} \otimes \Delta) \subset W \operatorname{Dom}(\hat{F} \otimes F) .
$$

The right hand side of the second equality in (2.10) shows that the domain of $\hat{F} \otimes F$ is invariant under $W$ and hence we have $(\hat{S} \otimes S) W^{*} \operatorname{Dom}(\hat{\Delta} \otimes \Delta) \subset \operatorname{Dom}(\hat{F} \otimes F)$. Therefore (2.13) together with the first equality in (2.10) implies

$$
\hat{\Delta} \otimes \Delta \subset W(\hat{F} \otimes F)(\hat{S} \otimes S) W^{*}=W(\hat{\Delta} \otimes \Delta) W^{*} .
$$

Since the range of the self-adjoint operator $\hat{\Delta} \otimes \Delta$ is dense in $\mathfrak{S} \otimes \mathfrak{S}$, so is the range of $W$. Thus $W$ is unitary and $\hat{\Delta} \otimes \Delta=W(\hat{\Delta} \otimes \Delta) W^{*}$.

(iii) According to (2.10) and (2.11),

$$
W=(\hat{J} \otimes J)(\hat{\Delta} \otimes \Delta)^{1 / 2} W^{*}(\hat{\Delta} \otimes \Delta)^{-1 / 2}(\hat{J} \otimes J)=(\hat{J} \otimes J) W^{*}(\hat{J} \otimes J)
$$

holds on the domain of $\hat{S} \otimes S$. Since both sides are bounded, the assertion holds. QED

Theorem 2.17. (i) The involutive algebra $\hat{\mathfrak{U}}=\hat{\eta}(\mathfrak{b})$ is a left Hilbert algebra.

(ii) The representation $\hat{\pi}_{\ell}$ of $\hat{\mathfrak{U}}$ coincides with the Fourier transform $\hat{\pi}$ in the sense that $\hat{\pi}_{\ell}(\hat{\eta}(\phi))=\hat{\pi}(\phi)$ for $\phi \in \mathfrak{b}$.

(iii) The von Neumann algebra $\hat{M}$ is generated by $\{\hat{\pi}(\phi): \phi \in \mathfrak{b}\}$.

Proof. Assertion (ii) is immediate from

$$
\hat{\pi}_{\rho}(\hat{\eta}(\phi)) \hat{\eta}(\psi)=\hat{\eta}(\phi) \hat{\eta}(\psi)=\hat{\eta}(\phi * \psi)=\hat{\pi}(\phi) \hat{\eta}(\psi) \text { for } \phi, \psi \in \mathfrak{b} .
$$

The continuity of the representation $\hat{\pi}_{\ell}(\xi): \eta \rightarrow \xi \eta$ and the closability of the left involution: $\xi \rightarrow \xi^{\#}$ were proved by Lemmas 2.8 and 2.13 , respectively. Since $\hat{\pi}(\phi)^{*}=\hat{\pi}\left(\phi^{\#}\right)$ for $\phi \in \mathfrak{b}$, the equality $(\xi \eta \mid \zeta)=\left(\eta \mid \xi^{\#} \zeta\right)$ follows from assertion (ii). It remains to show the density of $\hat{\mathfrak{A}}^{2}$ in $\hat{\mathfrak{U}}$ (or in $\mathfrak{\mathfrak { g }}$ ), where $\hat{\mathfrak{\mathfrak { A }}}^{2}$ is the linear span of elements of the form $\xi \eta$ with $\xi, \eta \in \hat{\mathfrak{U}}$. Suppose that $\xi \in \mathfrak{S}$ is orthogonal to $\hat{\mathfrak{U}}^{2}$. Then, for any $\phi, \psi \in \mathfrak{b},(\hat{\pi}(\phi) \xi \mid \hat{\eta}(\psi))=\left(\xi \mid \hat{\eta}\left(\phi^{\#} * \psi\right)\right)=0$. Therefore the density of $\hat{\mathfrak{U}}$ in $\mathfrak{S}$ implies $\hat{\pi}(\phi) \xi=0$. Hence for any $x, y \in \mathfrak{a}_{0}$ and $\eta \in \mathfrak{S}$

$$
\left(\eta_{h}(x) \otimes \xi \mid W\left(\eta_{h}(y) \otimes \eta\right)\right)=\left(\hat{\pi}\left(\omega_{\lambda, y}\right) \xi \mid \eta\right)=0
$$

by Lemma 2.10 . Since $W$ is unitary by Theorem $2.16, \eta_{h}(x) \otimes \xi=0$ for any $x \in \mathfrak{a}_{0}$. This implies $\xi=0$ and hence assertion (i) is proved.

Assertion (iii) follows from the density of $\mathfrak{b}$ in the predual space $M_{*}$. 
Due to this theorem, we are allowed to conclude that $\hat{\Delta}$ and $\hat{J}$ are the modular operator and the modular conjugation operator for the left Hilbert algebra $\hat{\mathfrak{U}}$.

\section{§3. Dual Woronowicz Algebra}

In the previous section, we constructed a left Hilbert algebra $\hat{\mathfrak{I}}=\hat{\eta}(\mathfrak{b})$ from the Woronowicz algebra $(M, \delta, R, \tau, h)$ and denoted the associated von Neumann algebra by $\hat{M}$. This section is devoted to construct the dual Woronowicz algebra $(\hat{M}, \hat{\delta}, \hat{R}, \hat{\tau}, \hat{h})$ which corresponds to the convolution algebra for the given "quantum group".

Let $\hat{W}$ denote the unitary $\sigma\left(W^{*}\right)$ on $\mathfrak{S} \otimes \mathfrak{S}$, where $\sigma$ is the flip automorphism on $\mathscr{L}(\mathfrak{S})$. Then the pentagonal relation for $\hat{W}$

$$
\hat{W}_{12} \hat{W}_{23}=\hat{W}_{23} \hat{W}_{13} \hat{W}_{12}
$$

follows from that for $W$ given by (2.3).

Proposition 3.1. Let $\hat{\delta}(x)=\hat{W}(1 \otimes x) \hat{W}^{*}$ for $x \in \hat{M}$. Then $\hat{\delta}$ is a coassociative coproduct on $\hat{M}$.

Proof. As $\hat{W} \in \hat{M} \otimes M$ by Lemma $2.10, \hat{\delta}(x) \in \hat{M} \otimes \mathscr{L}(\mathfrak{S})$. The pentagonal relation (3.1) for $\hat{W}$ guarantees the coassociativity of $\hat{\delta}$. It remains to show that $\hat{\delta}(x) \in \hat{M} \otimes \hat{M}$. For the proof of this fact, it suffices to show that each element $W^{*}(\hat{\pi}(\phi) \otimes 1) W$ for $\phi \in M_{*}$ commutes with $y \otimes 1$ for $y \in \hat{M}^{\prime}$, where $\hat{\pi}$ is the Fourier transform of $M_{*}$ to $\hat{M}$ defined by Definition 2.9. For $\xi, \eta \in \mathfrak{S} \otimes$ and $\xi^{\prime}, \eta^{\prime} \in$ we have

$$
\begin{aligned}
& \left((y \otimes 1) W^{*}\left(\hat{\pi}\left(\omega_{\xi^{\prime}, \eta^{\prime}}\right) \otimes 1\right) W \xi \mid \eta\right)=\left(\left(\hat{\pi}\left(\omega_{\xi^{\prime}, \eta^{\prime}}\right) \otimes 1\right) W \xi \mid W\left(y^{*} \otimes 1\right) \eta\right) \\
= & \left(\left(W^{*} \otimes 1\right)(1 \otimes W)\left(\xi^{\prime} \otimes \xi\right) \mid(1 \otimes W)\left(1 \otimes y^{*} \otimes 1\right)\left(\eta^{\prime} \otimes \eta\right)\right) \quad \text { (by Lemma 2.10) } \\
= & \left(\operatorname{Ad}_{1 \otimes W}\left(W^{*} \otimes 1\right)\left(\xi^{\prime} \otimes \xi\right) \mid\left(1 \otimes y^{*} \otimes 1\right)\left(\eta^{\prime} \otimes \eta\right)\right) .
\end{aligned}
$$

Since $\operatorname{Ad}_{1 \otimes W^{\prime}}\left(W^{*} \otimes 1\right)=W_{12}^{*} W_{13}^{*}$ by the pentagonal relation $(2.3), W_{12}^{*} W_{13}^{*}$ commutes with $1 \otimes y \otimes 1$ for $y \in \hat{M}^{\prime}$. Hence

$$
\begin{aligned}
\left((y \otimes 1) W^{*}\right. & \left.\left(\hat{\pi}\left(\omega_{\xi^{\prime}, \eta^{\prime}}\right) \otimes 1\right) W \xi \mid \eta\right) \\
& =\left(\operatorname{Ad}_{1 \otimes W^{\prime}}\left(W^{*} \otimes 1\right)\left(\xi^{\prime} \otimes(y \otimes 1) \xi\right) \mid \eta^{\prime} \otimes \eta\right) \\
& =\left(W^{*}\left(\hat{\pi}\left(\omega_{\xi^{\prime}, \eta^{\prime}}\right) \otimes 1\right) W(y \otimes 1) \xi \mid \eta\right),
\end{aligned}
$$

where the last equality follows from (3.2) by replacing $y$ and $\xi$ by 1 and $(y \otimes 1) \xi$, respectively. Since the set of finite linear combinations of $\omega_{\xi^{\prime}, \eta^{\prime}}$ 's is dense 
the predual space $M_{*}$ and $\left\{\hat{\pi}(\phi): \phi \in M_{*}\right\}^{\prime \prime}=\hat{M}$, we find that $\hat{\delta}(x)$ belongs to $\hat{M} \otimes \hat{M}$.

QED

By means of this coproduct we define a product $\theta_{1} * \theta_{2}$ in the predual space $\hat{M}_{*}$ of $\hat{M}$ by

$$
\theta_{1} * \theta_{2}=\left(\theta_{1} \otimes \theta_{2}\right) \circ \hat{\delta} \text { for } \theta_{1}, \theta_{2} \in \hat{M}_{*}
$$

Thus the predual space $\hat{M}_{*}$ turns out to be an algebra which is associative due to the coassociativity of $\hat{\delta}$.

Lemma 3.2. (i) The linear mapping: $\eta_{h}(x) \in \mathfrak{A}_{0} \rightarrow \eta_{h}\left(R \circ \tau_{-l / 2}(x)\right) \in \mathfrak{A}_{0}$ is closable. The closure $K$ satisfies $\rho^{-1} \Delta K \subset K^{*}$.

(ii) Let $K=U|K|$ be the polar decomposition. Then the followings hold:

a) $K^{2}=H$ and $|K|^{2}$ is the closure of $\rho^{-1} \Delta H$.

b) $U=\hat{J} J$ and $\hat{J} U \hat{\eta}(\phi)=\hat{\eta}\left(\phi^{*} \circ \sigma_{-t / 2}^{h}\right)$ for any $\left\{\sigma_{t}^{h}\right\}$ entire analytic $L^{2}$ bounded $\phi \in M_{*}$.

c) $\hat{J} H \hat{J}=H^{-1}$.

Proof. (i) For any $x$ and $y$ in $a_{0}$ we find that

$$
\begin{aligned}
\left(\eta_{h}\left(R \circ \tau_{-l / 2}(x)\right) \mid \eta_{h}(y)\right) & =h\left(y^{*} R \circ \tau_{-l / 2}(x)\right) \\
& =h \circ R\left(x R \circ \tau_{\imath / 2}\left(y^{*}\right)\right) \\
& =\left(J \rho^{-1 / 2} J \eta_{h}\left(R\left(\tau_{-l / 2}(y)\right)^{*}\right) \mid J \rho^{-1 / 2} J \eta_{h}\left(x^{*}\right)\right) \\
& =\left(\rho^{-1 / 2} \Delta^{1 / 2} \eta_{h}(x) \mid \rho^{-1 / 2} \Delta^{1 / 2} \eta_{h}\left(R\left(\tau_{-t / 2}(y)\right)\right)\right. \\
& =\left(\eta_{h}(x) \mid \rho^{-1} \Delta \eta_{h}\left(R\left(\tau_{-l / 2}(y)\right)\right) .\right.
\end{aligned}
$$

Since $\rho^{-t t} \Delta^{t t} \eta_{h}(y)=\eta_{h}\left(\sigma_{t}^{h \circ R}(y)\right)$, the mapping: $\eta_{h}(x) \rightarrow \eta_{h}\left(R \circ \tau_{-l / 2}(x)\right) \quad$ is closable. Denote the closure by $K$. Then $\eta_{h}(y) \in \operatorname{Dom}\left(K^{*}\right)$ and $K^{*} \eta_{h}(y)$ $=\rho^{-1} \Delta \eta_{h}\left(R \circ \tau_{-t / 2}(y)\right)$ for $y \in \mathfrak{a}_{0}$. Since $\mathfrak{a}_{0}$ is a core for $K, \rho^{-1} \Delta K \subset K^{*}$.

(ii) a) Let $K=U|K|$ be the polar decomposition. Since $K$ is invertible, $U$ is unitary. It is easy to verify that $K^{2}$ is positive symmetric and $K^{2} \eta_{h}(x)=$ $\eta_{h}\left(\tau_{-l}(x)\right)=H \eta_{h}(x)$ for $x \in \mathfrak{a}_{0}$. Since $\mathfrak{A}_{0}$ is a core for $K^{2}$ and $H$, it follows that $K^{2}=H$. Hence $\left(\rho^{-1} \Delta H\right)^{-} \subset|K|^{2}$. Since both sides are self-adjoint, the closure of $\rho^{-1} \Delta H$ is $|K|^{2}$.

b) Let $x$ be any element in $a_{0}$. Then

$$
\begin{aligned}
U \eta_{h}(x) & =K \rho^{1 / 2} H^{-1 / 2} \Delta^{-1 / 2} \eta_{h}(x)=K \rho^{1 / 2} \eta_{h}\left(\tau_{t / 2} \circ \sigma_{t / 2}^{h}(x)\right) \\
& =K \eta_{h}\left(L_{-t / 2}\left(\tau_{t / 2} \circ \sigma_{t / 2}^{h}(x)^{*}\right)^{*}\right) \\
& =\eta_{h}\left(R \circ \tau_{-t / 2}\left(L_{-t / 2}\left(\tau_{t / 2} \circ \sigma_{t / 2}^{h}(x)^{*}\right)^{*}\right)\right) .
\end{aligned}
$$


Since

$$
\begin{aligned}
& R \circ \tau_{-t / 2}\left(L_{-i / 2}\left(\tau_{i / 2} \circ \sigma_{i / 2}^{h}(x)^{*}\right)^{*}\right)=R \circ \tau_{i / 2}\left(L_{-l / 2}\left(\tau_{i / 2} \circ \sigma_{t / 2}^{h}(x)^{*}\right)\right)^{*} \\
& =R \circ L_{-l / 2}\left(\sigma_{i / 2}^{h}(x)^{*}\right)^{*}=L_{i / 2} \circ R\left(\sigma_{i / 2}^{h}(x)\right), \quad(\text { by Lemma 2.2) }
\end{aligned}
$$

it follows that

$$
U \eta_{h}(x)=\eta_{h}\left(L_{i / 2} \circ R \circ \sigma_{i / 2}^{h}(x)\right)=J \rho^{-1 / 2} J \eta_{h}\left(R \circ \sigma_{i / 2}^{h}(x)\right) .
$$

Since $J \rho^{-1} J$ is the closure of $\hat{\Delta}^{-1} H$ by Lemma 2.14 , we find that

$$
U \eta_{h}(x)=\hat{\Delta}^{-1 / 2} H^{1 / 2} \eta_{h}\left(R \circ \sigma_{i / 2}^{h}(x)\right)=\hat{\Delta}^{-1 / 2} \eta_{h}\left(\tau_{-l / 2} \circ R\left(\sigma_{i / 2}^{h}(x)\right)\right) .
$$

Applying the operator $\hat{J}$ to both sides, we have

$$
\hat{J} U \eta_{h}(x)=\hat{F} \eta_{h}\left(\tau_{-i / 2} \circ R\left(\sigma_{i / 2}^{h}(x)\right)\right)=\eta_{h}\left(\sigma_{i / 2}^{h}(x)^{*}\right)=S \Delta^{-1 / 2} \eta_{h}(x)=J \eta_{h}(x) .
$$

Thus $\hat{J} U=J$ and so $U=\hat{J} J$.

Let $\phi$ be any $\left\{\sigma_{t}^{h}\right\}$ entire analytic $L^{2}$-bounded element in $M_{*}$. Then

$$
\begin{aligned}
& \left(J \hat{\eta}(\phi) \mid \eta_{h}(x)\right)=\left(J \eta_{h}(x) \mid \hat{\eta}(\phi)\right)=\left(\eta_{h}\left(\sigma_{i / 2}^{h}(x)^{*}\right) \mid \hat{\eta}(\phi)\right) \\
& =\overline{\phi\left(\sigma_{t / 2}^{h}(x)\right)}=\phi^{*}\left(\sigma_{-i / 2}^{h}\left(x^{*}\right)\right)=\left(\hat{\eta}_{h}\left(\phi^{*} \circ \sigma_{-t / 2}^{h}\right) \mid \eta_{h}(x)\right),
\end{aligned}
$$

which implies that $\hat{J} U \hat{\eta}(\phi)=J \hat{\eta}(\phi)=\hat{\eta}\left(\phi^{*} \circ \sigma_{-l / 2}^{h}\right)$.

c) Since $K H=K^{3}=H K$, we see that $|K| H=U^{*} K H=U^{*} H K=U^{*} H U|K|$. Since $|K|$ commutes with $H$ by a), $H=U^{*} H U$ on $|K| \operatorname{Dom}\left(K^{3}\right)$. Since $|K| \operatorname{Dom}\left(K^{3}\right)$ is a core for $U^{*} H U$, it follows that $U^{*} H U \subset H$ and hence that $U^{*} H U=H$. Since $U=\hat{J} J$ by b), we have $\hat{J} H \hat{J}=J H J=H^{-1}$.

Let $\hat{\pi}_{*}$ denote the inverse Fourier transform, i.e. the mapping of the predual space $\hat{M}_{*}$ to $M$ obtained as the restriction of the dual mapping of the Fourier transform $\hat{\pi}$ of $M_{*}$ to $\hat{M}$. Let $\hat{\mathfrak{U}}^{\prime}$ be the right Hilbert algebra associated with the left Hilbert algebra $\hat{\mathfrak{U}}$ constructed in Section 2 .

Lemma 3.3. (i) $\hat{\pi}_{*}(\theta)=(\mathrm{id} \otimes \theta)\left(W^{*}\right)=(\theta \otimes \mathrm{id})(\hat{W})$.

(ii) $\hat{\pi}_{*}\left(\theta_{1} * \theta_{2}\right)=\hat{\pi}_{*}\left(\theta_{2}\right) \hat{\pi}_{*}\left(\theta_{1}\right)$ for $\theta_{1}, \theta_{2} \in \hat{M}_{*}$.

Proof. (i) For any $\phi \in M_{*}$ we have

$$
\phi\left(\hat{\pi}_{*}(\theta)\right)=\theta(\hat{\pi}(\phi))=\theta\left((\phi \otimes \mathrm{id})\left(W^{*}\right)\right)=(\phi \otimes \theta)\left(W^{*}\right)=\phi\left((\mathrm{id} \otimes \theta)\left(W^{*}\right)\right) .
$$

(ii) Due to the pentagonal relation, we have 


$$
(\mathrm{id} \otimes \hat{\delta})\left(W^{*}\right)=\hat{W}_{23} W_{13}^{*} \hat{W}_{23}^{*}=W_{13}^{*} W_{12}^{*} .
$$

Applying id $\otimes \theta_{1} \otimes \theta_{2}$ to both sides, we get the proof of our assertion.

Proposition 3.4. Let $\hat{R}(x)=J x^{*} J$ for $x \in \hat{M}$. Then

(i) $\hat{R}(\hat{\pi}(\phi))=\hat{\pi}(\phi \circ R)$ for all $\phi \in M_{*}$; and

(ii) $\hat{R}$ is a unitary antipode on $(\hat{M}, \hat{\delta})$, i.e. $\hat{R}$ is a *-antiautomorphism of $\hat{M}$ with $\hat{R}^{2}=$ id and $\sigma \circ \hat{R} \circ \hat{\delta}=(\hat{R} \otimes \hat{R}) \circ \hat{\delta}$.

Proof. (i) Let $\phi$ be any element in $M_{*}$. First we consider the case where $\phi \in\left(M_{*}\right)_{\tau}$. If $\psi$ is $L^{2}$-bounded and entire analytic with respect to $\left\{\sigma_{t}^{h}\right\}$, then

$$
\begin{aligned}
J \hat{\pi}(\phi \circ R) \hat{\eta}(\psi) & =J \hat{\eta}((\phi \circ R) * \psi)=\hat{\eta}\left(((\phi \circ R) * \psi)^{*} \circ \sigma_{-i / 2}^{h}\right) \\
& =\hat{\eta}\left(\left((\phi \circ R)^{*} \otimes \psi^{*}\right) \circ \delta \circ \sigma_{-l / 2}^{h}\right) .
\end{aligned}
$$

The Kac-Takesaki operator $W$ commutes with $\hat{\Delta} \otimes \Delta$ by Theorem 2.16 and the deformation automorphism $\left\{\tau_{t}\right\}$ of $M$ is implemented by $\left\{\hat{\Delta}^{t t}\right\}$ by Corollary 2.14.1. Hence the modular automorphism $\left\{\sigma_{t}^{h}\right\}$ satisfies a formula

$$
\left(\tau_{t} \otimes \sigma_{t}^{h}\right) \circ \delta=\delta \circ \sigma_{t}^{h}, \quad t \in \mathbf{R} .
$$

Therefore, by (3.3), we have

$$
\begin{aligned}
J \hat{\pi}(\phi \circ R) \hat{\eta}(\psi) & =\hat{\eta}\left(\left((\phi \circ R)^{*} \circ \tau_{-l / 2}\right) *\left(\psi^{*} \circ \sigma_{-t / 2}^{h}\right)\right)=\hat{\pi}\left((\phi \circ R)^{*} \circ \tau_{-t / 2}\right) \hat{\eta}\left(\psi^{*} \circ \sigma_{-t / 2}^{h}\right) \\
& \left.=\hat{\pi}(\phi)^{*} J \hat{\eta}(\psi) . \quad \quad(\text { by Lemma } 3.2(\mathrm{ii}) . \mathrm{b})\right)
\end{aligned}
$$

Thus $\hat{R}(\hat{\pi}(\phi))=J \hat{\pi}(\phi)^{*} J=\hat{\pi}(\phi \circ R)$ for $\phi \in\left(M_{*}\right)_{\tau}$.

For a general element $\phi$ in $M_{*}$ we approximate $\phi$ by a sequence $\left\{\phi_{n}\right\}$ in $\left(M_{*}\right)_{\tau}$. Since $\|\hat{\pi}(\omega)\| \leq\|\omega\|$ for $\omega \in M_{*}$ by Lemma 2.8 , our assertion (i) is proved.

(ii) It is clear from (i) that $\hat{R}$ maps $\hat{M}$ onto itself. Hence it is a $*-$ antiautomorphism of $\hat{M}$ with $\hat{R}^{2}=\mathrm{id}$.

For any $\theta_{1}, \theta_{2} \in \hat{M}_{*}$ we have

$$
\begin{aligned}
&\left(\theta_{1} \otimes\right.\left.\theta_{2}\right)(\sigma \circ \hat{\delta} \circ \hat{R}(\hat{\pi}(\phi)))=\left(\theta_{2} * \theta_{1}\right)(\hat{\pi}(\phi \circ R)) \\
& \quad=(\phi \circ R)\left(\hat{\pi}_{*}\left(\theta_{2} * \theta_{1}\right)\right)=(\phi \circ R)\left(\hat{\pi}_{*}\left(\theta_{1}\right) \hat{\pi}_{*}\left(\theta_{2}\right)\right) \\
& \quad=\phi\left(R\left(\hat{\pi}_{*}\left(\theta_{2}\right)\right) R\left(\hat{\pi}_{*}\left(\theta_{1}\right)\right)\right) .
\end{aligned}
$$

Since, for any $\theta \in \hat{M}_{*}$ and $\psi \in M_{*}$

$$
\begin{aligned}
\psi\left(R\left(\hat{\pi}_{*}(\theta)\right)\right) & =(\psi \circ R)\left(\hat{\pi}_{*}(\theta)\right)=\theta(\hat{\pi}(\psi \circ R)) \\
& =\theta(\hat{R}(\hat{\pi}(\psi)))=\theta \circ \hat{R}(\hat{\pi}(\psi))=\psi\left(\hat{\pi}_{*}(\theta \circ \hat{R})\right),
\end{aligned}
$$


we have a formula for the inverse Fourier transform:

$$
R\left(\hat{\pi}_{*}(\theta)\right)=\hat{\pi}_{*}(\theta \circ \hat{R}) \text { for } \theta \in \hat{M}_{*} .
$$

Therefore, from (3.5), we have

$$
\begin{aligned}
& \left(\theta_{1} \otimes \theta_{2}\right)(\sigma \circ \hat{\delta} \circ \hat{R}(\hat{\pi}(\phi)))=\phi\left(\hat{\pi}_{*}\left(\theta_{2} \circ \hat{R}\right) \hat{\pi}_{*}\left(\theta_{1} \circ \hat{R}\right)\right), \\
& =\phi\left(\hat{\pi}_{*}\left(\left(\theta_{1} \circ \hat{R}\right) *\left(\theta_{2} \circ \hat{R}\right)\right)\right) \\
& =\left(\left(\theta_{1} \circ \hat{R}\right) *\left(\theta_{2} \circ \hat{R}\right)\right)(\hat{\pi}(\phi))=\left(\theta_{1} \otimes \theta_{2}\right)((\hat{R} \otimes \hat{R}) \circ \hat{\delta} \circ(\hat{\pi}(\phi))) .
\end{aligned}
$$

Thus $\hat{R}$ satisfies $\sigma \circ \hat{\delta} \circ \hat{R}=(\hat{R} \otimes \hat{R}) \circ \hat{\delta}$. Consequently, $\hat{R}$ is a unitary antipode on $(\hat{M}, \hat{\delta})$.

QED

Lemma 3.5. (i) If $\psi \in M_{*}$ is $L^{2}$-bounded, then $\psi \circ \sigma_{-t}^{h}$ is also $L^{2}$-bounded and $\Delta^{t t} \hat{\eta}(\psi)=\hat{\eta}\left(\psi \circ \sigma_{-t}^{h}\right) ;$ and

(ii) $\Delta^{t t} \hat{\pi}(\phi) \Delta^{-t t}=\hat{\pi}\left(\phi \circ \tau_{-t}\right)$ for all $\phi \in M_{*}$.

Proof. (i) For each $x \in \mathfrak{n}_{h}$ we have $\sigma_{t}^{h}\left(\mathfrak{n}_{h}\right)=\mathfrak{n}_{h}$ and

$\left(\Delta^{\prime t} \hat{\eta}(\psi) \mid \eta_{h}(x)\right)=\left(\hat{\eta}(\psi) \mid \eta_{h}\left(\sigma_{-t}^{h}(x)\right)\right)=\psi\left(\sigma_{-t}^{h}\left(x^{*}\right)\right)=\left(\hat{\eta}\left(\psi \circ \sigma_{-t}^{h}\right) \mid \eta_{h}(x)\right)$.

(ii) Let $\phi$ be any element in $M_{*}$. Then

$\Delta^{t t} \hat{\pi}(\phi) \Delta^{-t t} \hat{\eta}(\psi)=\Delta^{t t} \hat{\pi}(\phi) \hat{\eta}\left(\psi \circ \sigma_{t}^{h}\right)=\Delta^{t t} \hat{\eta}\left(\phi *\left(\psi \circ \sigma_{t}^{h}\right)\right)=\hat{\eta}\left(\left(\phi *\left(\psi \circ \sigma_{t}^{h}\right)\right) \circ \sigma_{-t}^{h}\right)$. Since $\left(\tau_{t} \otimes \sigma_{t}^{h}\right) \circ \delta=\delta \circ \sigma_{t}^{h} \quad$ by $\quad(3.4)$, it follows that $\left(\phi *\left(\psi \circ \sigma_{t}^{h}\right)\right) \circ \sigma_{-t}^{h}$ $=\left(\phi \circ \tau_{-t}\right) * \psi$ and hence that

$$
\Delta^{t t} \hat{\pi}(\phi) \Delta^{-t t} \hat{\eta}(\psi)=\hat{\eta}\left(\left(\phi \circ \tau_{-t}\right) * \psi\right)=\hat{\pi}\left(\phi \circ \tau_{-t}\right) \hat{\eta}(\psi)
$$

Proposition 3.6. Let $\hat{\tau}_{t}(x)=H^{t t} x H^{-t t}$ for $x \in \hat{M}$ and $t \in \mathbb{R}$. Then

(i) $\hat{\tau}_{t}(\hat{\pi}(\phi))=\hat{\pi}\left(\phi \circ \tau_{-t}\right)$ for all $\phi \in M_{*}$; and

(ii) $\left\{\hat{\tau}_{t}\right\}$ is a deformation automorphism of $(\hat{M}, \hat{\delta}, \hat{R})$, i.e. it is a one parameter $*$-automorphism group with $\hat{\delta} \circ \hat{\tau}_{t}=\left(\hat{\tau}_{t} \otimes \hat{\tau}_{t}\right) \circ \hat{\delta}$ and $\hat{\tau}_{t} \circ \hat{R}=\hat{R} \circ \hat{\tau}_{t}$ for $t \in \mathbf{R}$.

Proof. (i) Let $\psi \in M_{*}$ be any $L^{2}$-bounded element which is entire analytic with respect to $\left\{\sigma_{t}^{h}\right\}$. Then for any $x \in \mathfrak{n}_{h}$ we have

$$
\left(H^{t} \hat{\eta}(\psi) \mid \eta_{h}(x)\right)=\left(\hat{\eta}(\psi) \mid \eta_{h}\left(\tau_{-t}(x)\right)\right)=\psi\left(\tau_{-t}\left(x^{*}\right)\right)=\left(\hat{\eta}\left(\psi \circ \tau_{-t}\right) \mid \eta_{h}(x)\right), t \in \mathbb{R} .
$$

Hence $H^{t} \hat{\eta}(\psi)=\hat{\eta}\left(\psi \circ \tau_{-t}\right)$. Let $\phi$ be any element in $M_{*}$. Then 


$$
\begin{aligned}
\hat{\tau}_{t}(\hat{\pi}(\phi)) \hat{\eta}(\psi) & =H^{i t} \hat{\pi}(\phi) H^{-t t} \hat{\eta}(\psi)=H^{i t} \hat{\pi}(\phi) \hat{\eta}\left(\psi \circ \tau_{t}\right) \\
& =H^{t t} \hat{\eta}\left(\phi *\left(\psi \circ \tau_{t}\right)\right)=\hat{\eta}\left(\left(\phi *\left(\psi \circ \tau_{t}\right)\right) \circ \tau_{-t}\right) .
\end{aligned}
$$

Since $\delta \circ \tau_{t}=\left(\tau_{t} \otimes \tau_{t}\right) \circ \delta$, we have $\left(\phi *\left(\psi \circ \tau_{t}\right)\right) \circ \tau_{-t}=\left(\phi \circ \tau_{-t}\right) * \psi$ and hence

$$
\hat{\tau}_{t}(\hat{\pi}(\phi)) \hat{\eta}(\psi)=\hat{\eta}\left(\left(\phi \circ \tau_{-t}\right) * \psi\right)=\hat{\pi}\left(\phi \circ \tau_{-t}\right) \hat{\eta}(\psi) .
$$

(ii) Taking (i) together with Lemma 3.5 gives us $\operatorname{Ad}_{\Delta^{t t}}=\operatorname{Ad}_{H^{u t}}$ on $\hat{M}$. On the other hand, $\operatorname{Ad}_{\hat{\Delta}^{t}}=\operatorname{Ad}_{H^{u}}$ on $M$ by Lemma 2.14. Moreover, $W \in M \otimes \hat{M}$ and $W$ commutes with $\hat{\Delta} \otimes \Delta$ by Theorem 2.16. This implies that the operator $W$ commutes with $(H \otimes H)^{t t}$ and hence $\hat{\delta}_{0} \circ \hat{\tau}_{t}=\left(\hat{\tau}_{t} \otimes \hat{\tau}_{t}\right) \circ \hat{\delta}$ for $t \in \mathbf{R}$.

The commutativity of $\hat{\tau}_{t}$ and the unitary antipode $\hat{R}$ follows immediately from that of $\tau_{t}$ and $R$ :

$$
\hat{\tau}_{t} \circ \hat{R}(\hat{\pi}(\phi))=\hat{\pi}\left(\phi \circ R \circ \tau_{t}\right)=\hat{\pi}\left(\phi \circ \tau_{-t} \circ R\right)=\hat{R} \circ \hat{\tau}_{t}(\hat{\pi}(\phi)) .
$$

QED

Corollary 3.6.1. (i) The deformation automorphism $\left\{\hat{\tau}_{t}\right\}$ is implemented by $\left\{\Delta^{t t}\right\}$ as well as $\left\{H^{t t}\right\}$.

(ii) The Kac-Takesaki operator $W$ commutes with the operator $H \otimes H$.

Combining the above argument with Lemmas 2.14 and 3.2, we have

Corollary 3.6.2. The following formulas hold:

(i) $J \hat{\eta}(\phi)=\hat{\eta}\left(\phi^{*} \circ \sigma_{-l / 2}^{h}\right)$ and $\Delta^{u t} \hat{\eta}(\phi)=\hat{\eta}\left(\phi \circ \sigma_{-t}^{h}\right)$ for $\phi \in \mathfrak{b}$;

(ii) $\hat{\Delta}^{t} \hat{\eta}(\phi)=\hat{\eta}\left(\left(\phi \rho^{t t}\right) \circ \tau_{-t}\right)$ and $\hat{J} \hat{\eta}(\phi)=\hat{\eta}\left(\left(\rho^{-1 / 2} \phi^{*}\right) \circ R \circ \tau_{-l}\right)$ for $\phi \in \mathfrak{b}$;

(iii) $\hat{J} \eta_{h}(x)=\eta_{h}\left(L_{t / 2} \circ R\left(x^{*}\right)\right)$ and $J \eta_{h}(x)=\eta_{h}\left(\sigma_{-t / 2}\left(x^{*}\right)\right)$ for $x \in \mathfrak{a}_{0}$; and

(iv) $\hat{J} J=J \hat{J}$.

Here the notation $\rho^{-1 / 2} \phi^{*}=\left(\phi \rho^{-1 / 2}\right)^{*}$ for $\phi \in \mathfrak{b}$ will be explained in Section 4 .

For the proof of assertion (iv), we have to use the formula $\sigma_{t}^{h \circ R} \circ R=R \circ \sigma_{-t}^{h}$, which is shown by using the KMS condition.

Let $\hat{\mathfrak{A}}^{\prime}$ denote the right Hilbert algebra associated with the left Hilbert algebra $\hat{\mathfrak{U}}$ obtained in Section 2 . Then $\hat{\pi}_{\ell}\left(\hat{\mathfrak{U}}^{2}\right)$ and $\hat{\pi}_{r}\left(\left(\hat{\mathfrak{U}}^{\prime}\right)^{2}\right)$ are $\sigma$-weakly dense *-subalgebras of $\hat{M}$ and $\hat{M}^{\prime}$, respectively, where $\hat{\mathfrak{U}}^{2}$ (resp. $\left.\left(\hat{\mathfrak{U}}^{\prime}\right)^{2}\right)$ is the linear span of elements of the form $\xi \eta$ for $\xi, \eta \in \hat{\mathfrak{U}}$ (resp. $\hat{\mathfrak{U}}^{\prime}$ ).

Lemma 3.7. (i) The set $\left(\hat{\mathfrak{U}}^{\prime}\right)^{2}$ is contained in $\eta_{h}\left(\mathfrak{n}_{h}\right)$.

(ii) If $\phi$ is the restriction of $\omega_{\xi^{\prime}, \eta^{\prime}}$ to $\hat{M}$ for $\xi^{\prime}, \eta^{\prime} \in \hat{\mathfrak{A}}^{\prime}$, then $\hat{\pi}_{*}(\phi)^{*} \in \mathfrak{n}_{h}$ and $\eta_{h}\left(\hat{\pi}_{*}(\phi)^{*}\right)=\eta^{\prime} \xi^{\prime b}$, where the superscript $b$ is the right involution of $\hat{\mathfrak{A}}^{\prime}$. 
Proof. (i) Let $\mathfrak{A}^{\prime}$ be the right Hilbert algebra associated with $\mathfrak{A}_{h}$. For any $\xi, \eta \in \mathfrak{U}^{\prime}$ and $\xi^{\prime}, \eta^{\prime} \in \hat{\mathfrak{A}}^{\prime}$ we have

$$
\begin{aligned}
& \left(\xi \otimes \xi^{\prime} \mid W\left(\eta \otimes \eta^{\prime}\right)\right)=\left(\hat{\pi}\left(\omega_{\xi, \eta}\right) \xi^{\prime} \mid \eta^{\prime}\right)=\left(\hat{\pi}_{r}\left(\xi^{\prime}\right) \hat{\eta}\left(\omega_{\xi, \eta}\right) \mid \eta^{\prime}\right) \\
& =\left(\xi \eta^{b} \mid \hat{\pi}_{r}\left(\xi^{\prime}\right)^{*} \eta^{\prime}\right)=\left(\pi_{r}(\eta)^{*} \xi \mid \eta^{\prime} \xi^{\prime b}\right)=\left(\xi \mid \pi_{r}(\eta) \eta^{\prime} \xi^{\prime b}\right),
\end{aligned}
$$

where the superscript $b$ in the fourth inner product is the right involution of $\mathfrak{a}^{\prime}$ and where $\hat{\pi}_{r}$ and $\pi_{r}$ are the representations of the right Hilbert algebras $\hat{\mathfrak{A}}^{\prime}$ and $\mathfrak{A}^{\prime}$, respectively. Hence $\left\|\pi_{r}(\eta) \eta^{\prime} \xi^{\prime}\right\| \leq\left\|\xi^{\prime}\right\|\left\|\eta^{\prime}\right\|\|\eta\|$ and $\eta^{\prime} \xi^{\prime b}$ is left bounded. There exist then an operator $x \in M$ such that $x \eta=\pi_{r}(\eta) \eta^{\prime} \xi^{\prime b}$ for $\eta \in \mathfrak{A}^{\prime}$. Therefore $x \in \mathfrak{n}_{h}$ and $\eta_{h}(x)=\eta^{\prime} \xi^{\prime b}$.

(ii) From the above equalities it follows that

$$
(\xi \mid x \eta)=\left(\hat{\pi}\left(\omega_{\xi, \eta}\right) \xi^{\prime} \mid \eta^{\prime}\right)=\left(\hat{\pi}_{*}\left(\omega_{\xi^{\prime}, \eta^{\prime}}\right) \xi \mid \eta\right) .
$$

Let $\phi$ be the restriction of $\omega_{\xi^{\prime}, \eta^{\prime}}$ to $\hat{M}$. Then $x^{*}=\hat{\pi}_{*}(\phi)$. Hence $\eta_{h}\left(\hat{\pi}_{*}(\phi)^{*}\right)$ $=\eta^{\prime} \xi^{\prime b}$ by (i).

QED

Let $\hat{h}$ denote the canonical weight on $\hat{M}$ associated with the left Hilbert algebra $\hat{\mathfrak{U}}$ :

$$
\hat{h}\left(\hat{\pi}_{\ell}(\xi)^{*} \hat{\pi}_{\ell}(\xi)\right)=\left\{\begin{array}{cl}
(\xi \mid \xi) & \text { if } \xi \in \hat{\mathfrak{U}}^{\prime \prime} \\
\infty & \text { otherwise }
\end{array}\right.
$$

where $\hat{\pi}_{\ell}$ is the representation of the achieved left Hilbert algebra $\hat{\mathfrak{U}}^{\prime \prime}$. Thus $\hat{\pi}_{\ell}(\xi)=\hat{\pi}(\phi)$ and $\hat{h}\left(\hat{\pi}_{\ell}(\xi)^{*} \hat{\pi}_{\ell}(\xi)\right)=\|\hat{\eta}(\phi)\|^{2}$ for $\xi=\hat{\eta}(\phi) \in \hat{\mathfrak{U}}$. In what follows we identify the underlying Hilbert space $\mathfrak{S}_{\hat{h}}$ of the GNS-construction $\left\{\pi_{\hat{h}}, \mathfrak{S}_{\hat{h}}, \eta_{\hat{h}}\right\}$ for $(\hat{M}, \hat{h})$ with the original Hilbert space $\mathfrak{S}=\mathfrak{S}_{\hat{h}}$ through the identification $\eta_{\hat{h}}(\hat{\pi}(\phi))=\hat{\eta}(\phi)$, where $\hat{\pi}$ is the Fourier transform of the algebra $M_{*}$ to $\hat{M}$. Hence $\pi_{\hat{h}}(\hat{\pi}(\phi))=\hat{\pi}(\phi)$. It is known that the weight $\hat{h}$ is faithful, semi-finite and normal and that $\mathfrak{U}_{\hat{h}}^{\prime}=\hat{\mathfrak{A}}^{\prime}$ and $\mathfrak{U}_{\hat{h}}=\hat{\mathfrak{A}}^{\prime \prime}$.

Proposition 3.8. (i) The operator $\hat{W}$ is the Kac-Takesaki operator, that is, it satisfies

$$
\hat{W} \eta_{\hat{h} \otimes \hat{h}}(x \otimes y)=\eta_{\hat{h} \otimes \hat{h}}(\hat{\delta}(y)(x \otimes 1)), \quad x, y \in \mathfrak{n}_{\hat{h}} .
$$

(ii) The weight $\hat{h}$ is left invariant:

$$
(\mathrm{id} \otimes \hat{h})(\hat{\delta}(z))=\hat{h}(z) 1, \quad z \in \hat{M}_{+} .
$$

Proof. (i) Let $\phi$ be any $L^{2}$-bounded element in $M_{*}$ and let $\xi_{1}, \eta_{1}, \xi_{2}$ and $\eta_{2}$ be elements in the right Hilbert algebra $\mathfrak{a}_{\hat{h}}^{\prime}$ corresponding to $\mathfrak{U}_{\hat{h}}$. Then $\hat{\pi}_{*}\left(\omega_{\xi_{,}, \eta}\right)^{*} \in \mathfrak{n}_{h}$ by Lemma 3.7 and 


$$
\begin{aligned}
& \left(\hat{\delta}(\hat{\pi}(\phi))\left(\xi_{1} \otimes \xi_{2}\right) \mid \eta_{1} \otimes \eta_{2}\right)=\left(\omega_{\xi_{1}, \eta_{1}} \otimes \omega_{\xi_{2}, \eta_{2}}\right)(\hat{\delta}(\hat{\pi}(\phi))) \\
& =\phi\left(\hat{\pi}_{*}\left(\omega_{\xi_{1}, \eta_{1}} * \omega_{\xi_{2}, \eta_{2}}\right)\right)=\phi\left(\hat{\pi}_{*}\left(\omega_{\xi_{2}, \eta_{2}}\right) \hat{\pi}_{*}\left(\omega_{\xi_{1}, \eta_{1}}\right)\right) \\
& =\left(\hat{\eta}\left(\hat{\pi}_{*}\left(\omega_{\xi_{1}, \eta_{1}}\right) \phi\right) \mid \eta_{h}\left(\hat{\pi}_{*}\left(\omega_{\xi_{2}, \eta_{2}}\right)^{*}\right)\right) \\
& =\left(\hat{\pi}_{*}\left(\omega_{\xi_{1}, \eta_{1}}\right) \hat{\eta}(\phi) \mid \eta_{2} \xi_{2}{ }^{b}\right) \quad \text { (by Lemma 3.7) } \\
& =\left(\hat{\pi}\left(\omega_{\hat{\eta}(\phi), \eta_{2} \xi_{2}{ }^{b}}\right) \xi_{1} \mid \eta_{1}\right) \\
& =\left(\hat{\eta}(\phi) \otimes \xi_{1} \mid W\left(\eta_{2} \xi_{2} b \otimes \eta_{1}\right)\right) \quad \text { (by Lemma 2.10.(ii)) } \\
& =\left(\hat{W}\left(\xi_{1} \otimes \hat{\eta}(\phi)\right) \mid \eta_{1} \otimes \eta_{2} \xi_{2} b\right) \\
& =\left(\left\{1 \otimes \hat{\pi}_{r}\left(\xi_{2}\right)\right\} \hat{W}\left(\xi_{1} \otimes \hat{\eta}(\phi)\right) \mid \eta_{1} \otimes \eta_{2}\right)
\end{aligned}
$$

where $\hat{\pi}_{r}$ is the representation of the right Hilbert algebra $\mathfrak{u}_{\hat{h}}^{\prime}$. Hence we have

$$
\hat{\delta}(\hat{\pi}(\phi))\left(\xi_{1} \otimes \xi_{2}\right)=\left\{1 \otimes \hat{\pi}_{,}\left(\xi_{2}\right)\right\} \hat{W}\left(\xi_{1} \otimes \hat{\eta}(\phi)\right)
$$

Since $\mathfrak{Z}_{\hat{h}}^{\prime}$ is dense in $\mathfrak{\mathfrak { g }}$, the element $\xi_{1}$ can be replaced by $\hat{\pi}_{r}(\xi) \eta_{\hat{h}}(x)$ for $\xi \in \mathfrak{A}_{\hat{h}}^{\prime}$ and $x \in \mathfrak{n}_{\hat{h}}$. Since $\hat{W} \in \hat{M} \otimes M$, it commutes with $\hat{\pi}_{r}(\xi) \otimes 1$. Thus

(3.9) $\left\{\hat{\pi}_{r}(\xi) \otimes \hat{\pi}_{r}\left(\xi_{2}\right)\right\} \hat{W}\left(\eta_{\hat{h}}(x) \otimes \hat{\eta}(\phi)\right)=\left(1 \otimes \hat{\pi}_{r}\left(\xi_{2}\right)\right) \hat{W}\left(\hat{\pi}_{r}(\xi) \eta_{\hat{h}}(x) \otimes \hat{\eta}(\phi)\right)$

$$
=\hat{\delta}(\hat{\pi}(\phi))\left(\hat{\pi}_{r}(\xi) \eta_{\hat{h}}(x) \otimes \xi_{2}\right)=\hat{\delta}(\hat{\pi}(\phi))(x \otimes 1)\left(\xi \otimes \xi_{2}\right) .
$$

Let $y$ be any element in $\mathfrak{n}_{\hat{h}}$. Then the vector $\xi=\eta_{\hat{h}}(y)$ is left bounded. There exists then a sequence $\left\{\hat{\eta}\left(\phi_{n}\right)\right\} \subset \hat{\mathfrak{U}}$ such that $\left\|\hat{\eta}\left(\phi_{n}\right)-\xi\right\|^{2}+\left\|\hat{\Delta}^{1 / 2}\left(\hat{\eta}\left(\phi_{n}\right)-\xi\right)\right\|^{2}$ $\rightarrow 0,\left\|\hat{\pi}\left(\phi_{n}\right)\right\| \leq\|y\|$ and $\hat{\pi}\left(\phi_{n}\right)$ converges strongly to $\hat{\pi},(\xi)=y$. Therefore we can replace $\hat{\eta}(\phi)$ and $\hat{\pi}(\phi)$ in (3.9) by $\eta_{\hat{h}}(y)$ and $y$, respectively. Hence we have

$$
\left\{\hat{\pi}_{r}(\xi) \otimes \hat{\pi}_{r}\left(\xi_{2}\right)\right\} \hat{W} \eta_{\hat{h} \otimes \hat{h}}(x \otimes y)=\hat{\delta}(y)(x \otimes 1)\left(\xi \otimes \xi_{2}\right) .
$$

Whence $\hat{W} \eta_{\hat{h} \otimes \hat{h}}(x \otimes y)$ is left bounded and we obtain

$$
\left(\hat{\pi}, \otimes \hat{\pi}_{,}\right)\left(\hat{W} \eta_{\hat{h} \otimes \hat{h}}(x \otimes y)\right)=\hat{\delta}(y)(x \otimes 1) .
$$

Consequently, $\hat{W} \eta_{\hat{h} \otimes \hat{h}}(x \otimes y)=\eta_{\hat{h} \otimes \hat{h}}(\hat{\delta}(y)(x \otimes 1))$ for $x, y \in \mathfrak{n}_{\hat{h}}$.

(ii) By virtue of (i) we find that

$$
(\hat{h} \otimes \hat{h})\left(\left(x^{*} \otimes 1\right) \hat{\delta}\left(y^{*} y\right)(x \otimes 1)\right)=\hat{h}\left(x^{*} x\right) \hat{h}\left(y^{*} y\right) \text { for } x, y \in \mathfrak{n}_{\hat{h}} .
$$

Hence $(\operatorname{id} \otimes \hat{h})(\hat{\delta}(z))=\hat{h}(z) 1$ for $z \in \mathfrak{m}_{\hat{h}}^{+}$, where $\mathfrak{m}_{\hat{h}}$ is the set of all finite linear combinations of $y^{*} x$ 's for $x, y \in \mathfrak{n}_{\hat{h}}$. If $z \in M_{+}$and $z \notin \mathfrak{m}_{\hat{h}}$, then there exists an increasing net $\left\{z_{l}\right\} \subset \mathfrak{m}_{\hat{h}}^{+}$such that $z_{l}>z$. Hence

$(\mathrm{id} \otimes \hat{h})(\hat{\delta}(z))=\sup (\mathrm{id} \otimes \hat{h})\left(\hat{\delta}\left(z_{l}\right)\right)=\sup \hat{h}\left(z_{l}\right) 1$, 
which is divergent. Hence $(\mathrm{id} \otimes \hat{h})(\hat{\delta}(z))=\hat{h}(z) 1$ for all $z \in M_{+}$.

QED

The following two lemmas will be used to show that the intersection $M \cap \hat{M}$ is the set of scalar multiples of the identity in Proposition 3.11. This fact will be needed for the analysis of the automorphism group $\left\{\gamma_{t}\right\}$ which will be defined in Lemma 3.12.

Lemma 3.9. For $x \in M, R(x)=\hat{J} x^{*} \hat{J}$.

Proof. Recall that $\hat{F} \eta_{h}(x)=\eta_{h}\left(R \circ \tau_{-l / 2}\left(x^{*}\right)\right)$ for $x \in \mathfrak{a}_{0}$ by (2.6). For any $x$ and $y$ in $\mathfrak{a}_{0}$ we have

$$
R \circ \tau_{-i / 2}(x) \eta_{h}(y)=\eta_{h}\left(\left\{R \circ \tau_{-i / 2}(x)\right\} y\right)=\hat{F} \eta_{h}\left(x^{*} R \circ \tau_{-i / 2}\left(y^{*}\right)\right)=\hat{F} x^{*} \hat{F} \eta_{h}(y)
$$

Hence $R \circ \tau_{-t / 2}(x)$ is the closure of $\hat{F} x^{*} \hat{F}$ for any $x \in \mathfrak{a}_{0}$. Since $\hat{\Delta}$ is the closure of $J \rho J H$ by Lemma 2.14, it follows from (3.10) that, for any $\xi \in \mathfrak{A}_{0}=\eta_{h}\left(a_{0}\right)$,

$$
\hat{J} x^{*} \hat{J} \xi=\hat{J} x^{*} \hat{\Delta}^{-1 / 2} \hat{F} \xi=\hat{J} \hat{\Delta}^{-1 / 2} \hat{\Delta}^{1 / 2} x^{*} \hat{\Delta}^{-1 / 2} \hat{F} \xi=\hat{F} \tau_{-\imath / 2}\left(x^{*}\right) \hat{F} \xi=R(x) \xi
$$

Thus $R(x)=\hat{J} x^{*} \hat{J}$ for any $x \in \mathfrak{a}_{0}$. Since $\mathfrak{a}_{0}$ is $\sigma$-weakly dense in $M, R(x)=\hat{J} x^{*} \hat{J}$ for all $x \in M$.

Lemma 3.10. If $x \in M \cap \hat{M}$ is an entire analytic element for both the modular automorphism $\left\{\sigma_{t}^{\hat{h}}\right\}$ and the deformation automorphism $\left\{\hat{\tau}_{t}\right\}$, then $\mathfrak{a}_{\hat{h}} \hat{\tau}_{-l / 2}(x) \subset \mathfrak{a}_{\hat{h}}$ and $\eta_{\hat{h}}\left(y \hat{\tau}_{-i / 2}(x)\right)=R(x) \eta_{\hat{h}}(y)$ for $y \in \mathfrak{a}_{\hat{h}}$, where $\mathfrak{a}_{\hat{h}}=\left\{x \in \mathfrak{n}_{\hat{h}}: x^{*} \in\right.$ $\left.\mathfrak{n}_{\hat{h}}\right\}$.

Proof. Let $x$ be an entire analytic element in $M \cap \hat{M}$ for the modular automorphism $\left\{\sigma_{t}^{\hat{h}}\right\}$ as well as the deformation automorphism $\left\{\hat{\tau}_{t}\right\}$. It is known that $\mathfrak{a}_{\hat{h}}$ is a $(\hat{M})_{\sigma^{h}}$-bimodule, where $(\hat{M})_{\sigma^{h}}$ is the set of all entire analytic elements in $\hat{M}$ with respect to $\left\{\sigma_{t}^{\hat{h}}\right\}$. Since $\hat{\tau}_{-l / 2}(x)$ is entire analytic for $\left\{\sigma_{t}^{\hat{h}}\right\}, a_{\hat{h}} \hat{\tau}_{-t / 2}(x) \subset$ $\mathfrak{a}_{\hat{h}}$. Notice that

$$
\sigma_{t}^{\hat{h}}(x)=\hat{\Delta}^{t} x \hat{\Delta}^{-t t}=H^{t t} x H^{-t t}=\hat{\tau}_{t}(x),
$$

where we use the fact that $x$ is an element of $M$ for the second equality by Corollary 2.14.1. Since $x$ is entire analytic for $\left\{\sigma_{t}^{\hat{h}}\right\}$ and $\left\{\hat{\tau}_{t}\right\}, \sigma_{\alpha}^{\hat{h}}(x)=\hat{\tau}_{\alpha}(x)$ for $\alpha \in \mathbf{C}$ by analytic continuation. Since $\sigma_{t}^{\hat{h}}\left(x^{*}\right)=\hat{\tau}_{t}(x)^{*}, \sigma_{\alpha}^{\hat{h}}\left(x^{*}\right)=\hat{\tau}_{\bar{\alpha}}(x)^{*}$ by analytic continuation. Thus, for any $y \in \mathfrak{a}_{\hat{h}}$, we see that $\sigma_{-l / 2}^{\hat{h}}\left(\hat{\tau}_{-l / 2}(x)^{*}\right)$ $=\sigma_{-l / 2}^{\hat{h}}\left(\hat{\tau}_{t / 2}\left(x^{*}\right)\right)=x^{*}$ and hence 


$$
\begin{aligned}
\eta_{\hat{h}}\left(y \hat{\tau}_{-l / 2}(x)\right) & =\hat{S} \hat{\tau}_{-l / 2}(x)^{*} \hat{S} \eta_{\hat{h}}(y)=\hat{J} \sigma_{-l / 2}^{\hat{h}}\left(\hat{\tau}_{-l / 2}(x)^{*}\right) \hat{J} \eta_{\hat{h}}(y) \\
& =\hat{J} x^{*} \hat{J} \eta_{\hat{h}}(y)=R(x) \eta_{\hat{h}}(y) .
\end{aligned}
$$

QED

Proposition 3.11. $M \cap \hat{M}=\mathrm{C} 1$.

Proof. Let $x$ be an element in $M \cap \hat{M}$. Then $R(x)=\hat{J} x^{*} \hat{J}$ is an element in $M \cap \hat{M}^{\prime}$. Therefore $\delta(R(x))=\operatorname{Ad}_{W}(1 \otimes R(x))=1 \otimes R(x)$, for $W \in M \otimes \hat{M}$ by Theorem 2.16. Since $\sigma \circ \delta \circ R=(R \otimes R) \circ \delta$, we find that $\delta(x)=x \otimes 1$ or $W(1 \otimes x)=(x \otimes 1) W$. Since $\operatorname{Ad}_{\hat{\jmath} \otimes J}(W)=W^{*}$ by Theorem 2.16 , it follows that

$$
W(R(x) \otimes 1)=(1 \otimes \hat{R}(x)) W, \quad \hat{W}(\hat{R}(x) \otimes 1)=(1 \otimes R(x)) \hat{W} .
$$

First we consider the case where $x \in M \cap \hat{M}$ is entire analytic for the modular automorphism $\left\{\sigma_{t}^{\hat{h}}\right\}$ and the deformation automorphism $\left\{\hat{\tau}_{t}\right\}$. Then $R(x) \eta_{\hat{h}}(y)=\eta_{\hat{h}}\left(y \hat{\tau}_{-l / 2}(x)\right)$ for $y \in \mathfrak{a}_{\hat{h}}$ by Lemma 3.10. Therefore, for any $y, z \in \mathfrak{a}_{\hat{h}}$, we see that

$$
\begin{array}{rlr}
\eta_{\hat{h} \otimes \hat{h}}(\hat{\delta}(z)(\hat{R}(x) y \otimes 1)) & =\hat{W} \eta_{\hat{h} \otimes \hat{h}}(\hat{R}(x) y \otimes z) & \text { (by Proposition 3.8) } \\
& =\hat{W}(\hat{R}(x) \otimes 1) \eta_{\hat{h} \otimes \hat{h}}(y \otimes z) \\
& =(1 \otimes R(x)) \hat{W} \eta_{\hat{h} \otimes \hat{h}}(y \otimes z) \\
& =(1 \otimes R(x)) \eta_{\hat{h} \otimes \hat{h}}(\hat{\delta}(z)(y \otimes 1)) \quad \text { (by Proposition 3.8) } \\
& =\eta_{\hat{h} \otimes \hat{h}}\left(\hat{\delta}(z)\left(y \otimes \hat{\tau}_{-l / 2}(x)\right)\right) .
\end{array}
$$

The set $\mathfrak{a}_{\hat{h}}$ is $\sigma$-weakly dense in $\hat{M}$. Thus $\hat{R}(x) y \otimes 1=y \otimes \hat{\tau}_{-t / 2}(x)$ and so $\hat{R}(x) \otimes 1=1 \otimes \hat{\tau}_{-l / 2}(x)$. Hence $x \in \mathbf{C} 1$. Since each element in $M \cap \hat{M}$ can be approximated by entire analytic elements in $M \cap \hat{M}$ for $\left\{\sigma_{t}^{\hat{h}}\right\}$ and $\left\{\hat{\tau}_{t}\right\}, M \cap \hat{M}$ must be $\mathbf{C} 1$.

QED

Corollary 3.11.1. $M \cap \hat{M}^{\prime}=M^{\prime} \cap \hat{M}=M^{\prime} \cap \hat{M}^{\prime}=\mathrm{C} 1$.

Let $G$ be the set of all comultiplicative elements $\{x \in \hat{M}: \hat{\delta}(x)=x \otimes x, x \neq 0\}$. Owing to the discussion in $[20,23]$, it can be shown that $G$ is a subgroup of the unitary group of $M$ and $R(x)=x^{-1}$ for $x \in G$ by the above corollary. Since the set $\{x \in \hat{M}: \hat{\delta}(x)=x \otimes x\}$ is $\sigma$-weakly closed, the subgroup $G$ is locally compact. When the Woronowicz algebra is commutative, the dual Woronowicz algebra is cocommutative. Hence the predual $\hat{M}_{*}$ is a commutative Banach algebra with the spectrum $G$. 
Now we define the automorphisms $\left\{\gamma_{t}\right\}$ and $\left\{\hat{\gamma}_{t}\right\}$ for a Woronowicz algebra and its dual Woronowicz algebra, respectively.

Lemma 3.12. Let $\gamma_{t}$ and $\hat{\gamma}_{t}$ be the restrictions of $\operatorname{Ad}_{\rho^{t} J \rho^{\prime \prime} J}$ to $M$ and $\hat{M}$, respectively. Then

(i) $\left\{\gamma_{t}\right\}$ is a one parameter *-automorphism group of $M$ and $\left\{\operatorname{Ad}_{\rho^{\prime \prime}}\right\}$, $\left\{\operatorname{Ad}_{J \rho^{\prime \prime} J}\right\}$ and $\left\{\hat{\gamma}_{t}\right\}$ are one parameter $*$-automorphism group of $\hat{M}$;

(ii) $\delta\left(\rho^{i t}\right)=\rho^{i t} \otimes \rho^{i t}$ and

$$
\left(\gamma_{t} \otimes \gamma_{t}\right) \circ \delta=\delta \circ \gamma_{t}, \quad t \in \mathbf{R}
$$

(iii) $\hat{\gamma}_{t}(\hat{\pi}(\phi))=\hat{\pi}\left(\phi \circ \gamma_{-t}\right)$ for all $\phi \in M_{*}$ and $t \in \mathbf{R}$.

Proof. (i) It is clear that $\left\{\gamma_{t}\right\}$ is a one parameter *-automorphism group of $M$. Since $\hat{\Delta}$ is the closure of $J \rho J H$ by Lemma $2.14, \operatorname{Ad}_{J \rho^{\prime \prime} J}=\hat{\tau}_{t} \circ \sigma_{-t}^{\hat{h}}$ is a $*-$ automorphism of $\hat{M}$. Since $\hat{R}(x)=J x * J$ for $x \in \hat{M}, \operatorname{Ad}_{\rho^{t t}}=\hat{R} \circ \operatorname{Ad}_{J \rho^{t t} J} \circ \hat{R}$ is also a $*$-automorphism group of $\hat{M}$. Thus $\left\{\hat{\gamma}_{t}\right\}$ is a one parameter $*$-automorphism group of $\hat{M}$.

(ii) The left hand side of the equality

$$
\left(1 \otimes \rho^{i t}\right) \delta\left(\rho^{-t t}\right)=\left(\mathrm{id} \otimes \operatorname{Ad}_{\rho^{t}}\right)(W) W^{*}
$$

belongs to $M \otimes M$ and the right hand side belongs to $M \otimes \hat{M}$ by (i), for $W \in M \otimes \hat{M}$. Hence it belongs to $M \otimes \mathbf{C l}$ by Proposition 3.11. Therefore, for any fixed $t \in \mathbf{R}, \quad\left(1 \otimes \rho^{t t}\right) \delta\left(\rho^{-t t}\right)=v^{*} \otimes 1$ for some unitary $v$ in $M$; hence $\delta\left(\rho^{t t}\right)=v \otimes \rho^{t t}$. Replacing $\rho^{t t}$ by $R\left(\rho^{t t}\right)$ in the above argument, we see that $\delta\left(R\left(\rho^{t}\right)\right)=w \otimes R\left(\rho^{t}\right)$ for some unitary $w$ in $M$. Since $\sigma \circ \delta \circ R=(R \otimes R) \circ \delta$, we find that $R(w) \otimes \rho^{t t}=\rho^{t t} \otimes v$ and hence $R(w) \rho^{-t t} \otimes 1=1 \otimes v \rho^{-t t}$. Thus $v=\lambda(t) \rho^{i t}$ for some $\lambda(t) \in \mathbf{C}$, i.e. $\delta\left(\rho^{i t}\right)=\lambda(t) \rho^{i t} \otimes \rho^{t t}$. It is easy to see from the multiplicativity of the function $\lambda$ that $\lambda(t)=\mu^{t t}$ for some constant $\mu>0$. Since $R\left(\rho^{t t}\right)=\rho^{-t t}$ by $(1.1), \delta\left(\rho^{t}\right)=\delta\left(R\left(\rho^{-t t}\right)\right)=\sigma \circ(R \otimes R) \circ \delta\left(\rho^{-i t}\right)=\lambda^{-i t} \rho^{i t} \otimes \rho^{i t}$. Hence $\delta\left(\rho^{t t}\right)=\rho^{t t} \otimes \rho^{t t}$.

Since $\gamma_{t}=\operatorname{Ad}_{\rho^{\prime \prime}}$ on $M$, formula (3.11) is immediate from the above.

(iii) For any $L^{2}$-bounded $\psi$ and $x \in \mathfrak{n}_{h}$, we have

$$
\begin{gathered}
\left(\rho^{t t} J \rho^{t t} J \hat{\eta}(\psi) \mid \eta_{h}(x)\right)=\left(\hat{\eta}(\psi) \mid \rho^{-t t} J \rho^{-t t} J \eta_{h}(x)\right) \\
=\left(\hat{\eta}(\psi) \mid \eta_{h}\left(\gamma_{-t}(x)\right)\right)=\left(\hat{\eta}\left(\psi \circ \gamma_{-t}\right) \mid \eta_{h}(x)\right) .
\end{gathered}
$$

Hence we have a formula;

$$
\rho^{i t} J \rho^{\prime t} J \hat{\eta}(\psi)=\hat{\eta}\left(\psi \circ \gamma_{-t}\right) .
$$

Let $\phi$ be any element in $M_{*}$. Then $\left\{\phi *\left(\psi \circ \gamma_{t}\right)\right\} \circ \gamma_{-t}=\left(\phi \circ \gamma_{-t}\right) * \psi$ obtained by (3.11) in (ii) implies that 


$$
\begin{gathered}
\hat{\gamma}_{t}(\hat{\pi}(\phi)) \hat{\eta}(\psi)=\rho^{i t} J \rho^{i t} J \hat{\pi}(\phi) \hat{\eta}\left(\psi \circ \gamma_{t}\right)=\rho^{i t} J \rho^{i t} J \hat{\eta}\left(\phi *\left(\psi \circ \gamma_{t}\right)\right) \\
=\hat{\eta}\left(\left\{\phi *\left(\psi \circ \gamma_{t}\right)\right\} \circ \gamma_{-t}\right)=\hat{\eta}\left(\left(\phi \circ \gamma_{-t}\right) * \psi\right)=\hat{\pi}\left(\phi \circ \gamma_{-t}\right) \hat{\eta}(\psi)
\end{gathered}
$$

QED

Proposition 3.13. Let $\hat{h}$ be the Haar weight on $(\hat{M}, \hat{\delta}, \hat{R}, \hat{\tau})$. Then it is $\left\{\hat{\tau}_{t}\right\}-$ invariant as well as $\left\{\hat{\gamma}_{t}\right\}$-invariant.

Proof. The proof is the same for both automorphisms. We have only consider the $\left\{\hat{\tau}_{t}\right\}$-invariance of the Haar weight. Let $s$ be an arbitrarily fixed real number. Then $\hat{h} \circ \hat{\tau}_{s}$ is a faithful, semi-finite, normal weight on $\hat{M}$. For any $\hat{\eta}(\phi)$ in $\hat{\mathfrak{U}}$ we have

$$
\begin{gathered}
\hat{h} \circ \hat{\tau}_{s}\left(\hat{\pi}(\phi)^{*} \hat{\pi}(\phi)\right)=\hat{h}\left(\hat{\pi}\left(\phi \circ \pi_{-s}\right)^{*} \hat{\pi}\left(\phi \circ \tau_{-s}\right)\right)=\left(\hat{\eta}\left(\phi \circ \pi_{-s}\right) \mid \hat{\eta}\left(\phi \circ \pi_{-s}\right)\right) \\
=\left(H^{i s} \hat{\eta}(\phi) \mid H^{i s} \hat{\eta}(\phi)\right)=\hat{h}\left(\hat{\pi}(\phi)^{*} \hat{\pi}(\phi)\right) .
\end{gathered}
$$

Therefore $\hat{h} \circ \hat{\tau}_{s}=\hat{h}$ on a $\sigma$-weakly dense subalgebra $\hat{\pi}_{\ell}\left(\hat{\mathfrak{A}}^{2}\right)$ of $\hat{M}$, where $\hat{\mathfrak{A}}^{2}$ is the set of all finite linear combinations of $\xi \eta$ for $\xi, \eta \in \hat{\mathfrak{U}}$. Since $\sigma_{t}^{\hat{h}}\left(\hat{\pi}_{\ell}\left(\hat{\mathfrak{A}}^{2}\right)\right)=\hat{\pi}_{\ell}\left(\hat{\mathfrak{A}}^{2}\right)$, if we can show the $\left\{\sigma_{t}^{\hat{h}}\right\}$-invariance of $\hat{h} \circ \hat{\tau}_{s}$, then $\hat{h} \circ \hat{\tau}_{s}=\hat{h}$, namely, $\hat{h}$ is $\left\{\hat{\tau}_{s}\right\}$-invariant. However, the $\left\{\sigma_{t}^{\hat{h}}\right\}$-invariance of $\hat{h} \circ \hat{\tau}_{s}$ is clear from the commutativity of $\hat{\Delta}$ and $H$.

QED

For the proof of the commutativity of the weights $\hat{h}$ and $\hat{h} \circ R$, we will utilize the previous proposition.

Proposition 3.14. The Haar weight $\hat{h}$ commutes with the weight $\hat{h} \circ \hat{R}$.

Proof. Since $R\left(\rho^{t t}\right)=\rho^{-t t}$ by (1.1), it follows that $R \circ \gamma_{t}=\gamma_{t} \circ R$. Since $\hat{R}(\hat{\pi}(\phi))=\hat{\pi}(\phi \circ R)$ by Proposition 3.4 and $\hat{\gamma}_{t}(\hat{\pi}(\phi))=\hat{\pi}\left(\phi \circ \gamma_{-t}\right)$ by Lemma 3.12, it follows that $\hat{R} \circ \hat{\gamma}_{t}=\hat{\gamma}_{t} \circ \hat{R}$. Since $\hat{R}(\hat{\pi}(\phi))=\hat{\pi}(\phi \circ R)$ and $\hat{\tau}_{t}(\hat{\pi}(\phi))=\hat{\pi}\left(\phi \circ \tau_{-t}\right)$ by Proposition 3.6 , it follows that $\hat{R} \circ \hat{\tau}_{t}=\hat{\tau}_{t} \circ \hat{R}$. Since $\sigma_{t}^{\hat{h}}=\operatorname{Ad}_{J \rho^{-u J} J} \circ \hat{\tau}_{t}$ by Lemma 2.14 and $\hat{h}$ is $\left\{\hat{\tau}_{t}\right\}$-invariant by Proposition 3.13 , it follows that $\hat{h}$ is $\left\{\operatorname{Ad}_{J \rho^{\prime \prime} J}\right\}$-invariant. Since $\hat{\gamma}_{t}=\operatorname{Ad}_{\rho^{\prime \prime}} \circ \operatorname{Ad}_{J \rho^{\prime t} J}$ and $\hat{h}$ is $\left\{\hat{\gamma}_{t}\right\}$-invariant by Proposition 3.13, it follows that $\hat{h}$ is $\left\{\mathrm{Ad}_{\rho^{\prime \prime}}\right\}$-invariant. Since

$$
\hat{R} \circ \sigma_{t}^{\hat{h}}=\hat{R} \circ \operatorname{Ad}_{J \rho^{-t} J} \circ \hat{\tau}_{t}=\operatorname{Ad}_{\rho^{-t}} \circ \hat{R} \circ \hat{\tau}_{t}=\operatorname{Ad}_{\rho^{-t}} \circ \hat{\tau}_{t} \circ \hat{R}
$$

and $\hat{h}$ is $\left\{\operatorname{Ad}_{\rho^{-t t}} \circ \hat{\tau}_{t}\right\}$-invariant, we find that $\hat{h} \circ \hat{R} \circ \sigma_{t}^{\hat{h}}=\hat{h} \circ \hat{R}$, which means that $\hat{h}$ commutes with $\hat{h} \circ \hat{R}$.

QED

Remark. The Haar weight $\hat{h}$ is invariant under $\left\{\operatorname{Ad}_{\rho^{\prime \prime}}\right\}$ as well as $\left\{\operatorname{Ad}_{J \rho^{\prime \prime J}}\right\}$. 
The $L^{2}$-boundedness for elements in $\hat{M}_{*}$ is defined similarly as that for $\hat{M}_{*}$; i.e. the element $\omega \in \hat{M}_{*}$ is said to be $L^{2}$-bounded if there exists a positive constant $\lambda>0$ such that $\left|\omega\left(x^{*}\right)\right| \leq \lambda\left\|\eta_{\hat{h}}(x)\right\|$ for all $x \in \mathfrak{n}_{\hat{h}}$. Let $\hat{\hat{\eta}}$ be the linear mapping from $L^{2}$-bounded elements in $\hat{M}_{*}$ to $\mathfrak{S i t h} \omega\left(x^{*}\right)=\left(\hat{\eta}(\omega) \mid \eta_{\hat{h}}(x)\right)$ for $\omega \in \hat{M}_{*}$ and $x \in \mathfrak{n}_{\hat{h}}$. By using the boundedness of the Kac-Takesaki operator $\hat{W}$, it is shown that, if $\theta \in \hat{M}_{*}$, then, for any $L^{2}$-bounded $\omega \in \hat{M}_{*}$, the product $\theta * \omega \in \hat{M}_{*}$ is $L^{2}$-bounded and the bounded operator $\hat{\hat{\pi}}(\theta)$ on is defined by $\hat{\hat{\pi}}(\theta) \hat{\hat{\eta}}(\omega)=\hat{\hat{\eta}}(\theta * \omega)$ similarly as Definition 2.8. The mapping $\hat{\hat{\pi}}: \hat{M}_{*} \rightarrow \mathscr{L}(\mathfrak{S})$ is called the Fourier transform. The relation of the Fourier transform with the KacTakesaki operator is given by the same formula as in Lemma 2.10 :

$$
\left(\xi_{1} \otimes \xi_{2} \mid \hat{W}\left(\xi_{3} \otimes \xi_{4}\right)\right)=\left(\hat{\hat{\pi}}\left(\omega_{\xi_{1}, \xi_{3}}\right) \xi_{2} \mid \xi_{4}\right) .
$$

Let $\hat{\mathfrak{a}}_{0}$ be the entire analytic part of $\mathfrak{a}_{\hat{h}}$ with respect to four transformations $\left\{\sigma_{t}^{\hat{h}}\right\}$, $\left\{\sigma_{t}^{\hat{h} \circ \hat{R}}\right\},\left\{\hat{\tau}_{t}\right\}$ and $\left\{\hat{L}_{t}\right\}$, where $\hat{L}_{t} x=x \hat{\rho}^{\prime t}$ for $x \in \hat{M}$ and $\hat{\rho}$ is the Radon-Nikodym derivative of $\hat{h}$ with respect to $\hat{h} \circ \hat{R}$.

Lemma 3.15. (i) The Fourier transform $\hat{\hat{\pi}}$ of $\hat{M}_{*}$ to $\mathscr{L}($ in linear and multiplicative.

(ii) If $\theta \in \hat{M}_{*}$ is entire analytic with respect to $\left\{\hat{\tau}_{t}\right\}$, then $\hat{\hat{\pi}}(\theta)$ $=R \circ \tau_{-l / 2} \circ \hat{\pi}_{*}(\theta) \in M$ and $\hat{\hat{\pi}}\left(\theta^{\#}\right)=\hat{\hat{\pi}}(\theta)^{*}$, where $\theta^{\#}=\theta^{*} \circ \hat{R} \circ \hat{\tau}_{-t / 2}$.

Proof. (i) It is clear from the definition of the Fourier transform $\hat{\hat{\pi}}$.

(ii) Let $x \in \hat{\mathfrak{a}}_{0}$ and let $\theta^{\prime} \in \hat{M}_{*}$ be $L^{2}$-bounded and entire analytic with respect to $\left\{\hat{\tau}_{t}\right\}$. If $\xi$ and $\eta$ are elements in

$$
\begin{aligned}
& \left(\hat{\hat{\pi}}\left(\omega_{\xi, \eta}\right) \hat{\hat{\eta}}\left(\theta^{\prime}\right) \mid \eta_{\hat{h}}(x)\right)=\left(\xi \otimes \hat{\hat{\eta}}\left(\theta^{\prime}\right) \mid \hat{W}\left(\eta \otimes \eta_{\hat{h}}(x)\right)\right) \\
& =\left(W\left(\hat{\hat{\eta}}\left(\theta^{\prime}\right) \otimes \xi\right) \mid \eta_{\hat{h}}(x) \otimes \eta\right)=\left(\xi \mid \hat{\pi}\left(\omega_{\eta_{\hat{h}}(\lambda), \hat{\hat{\eta}}(\theta)}\right) \eta\right) \\
& =\omega_{\xi, \eta}\left(\hat{\pi}\left(\omega_{\eta_{\dot{h}^{(}(x), \hat{\eta}\left(\theta^{\prime}\right)}}\right)^{*}\right) .
\end{aligned}
$$

Since each element $\theta \in \hat{M}_{*}$ is written in terms of $\omega_{\xi, \eta}$ 's, we replace $\omega_{\xi, \eta}$ in the above equality by $\theta \in \hat{M}_{*}$. If $\phi \in M_{*}$, then, by Proposition 3.6(i),

$$
H^{t \prime} \eta_{\hat{h}}(\hat{\pi}(\phi))=H^{t t} \hat{\eta}(\phi)=\hat{\eta}\left(\phi \circ \tau_{-t}\right)=\eta_{\hat{h}}\left(\hat{\pi}\left(\phi \circ \tau_{-t}\right)\right)=\eta_{\hat{h}}\left(\hat{\tau}_{t}(\hat{\pi}(\phi))\right) .
$$

Hence we obtain $H^{t t} \eta_{\hat{h}}(x)=\eta_{\hat{h}}\left(\hat{\tau}_{t}(x)\right)$. If $y \in \mathfrak{n}_{\hat{h}}$, then

$$
\left(H^{\prime \prime} \hat{\hat{\eta}}(\theta) \mid \eta_{\hat{h}}(y)\right)=\left(\hat{\hat{\eta}}(\theta) \mid \eta_{\hat{h}}\left(\hat{\tau}_{-t}(y)\right)\right)=\theta\left(\hat{\tau}_{-t}(y)^{*}\right)=\left(\hat{\hat{\eta}}\left(\theta \circ \hat{\tau}_{-t}\right) \mid \eta_{\hat{h}}(y)\right) .
$$

Hence we obtain $H^{\prime \prime} \hat{\hat{\eta}}(\theta)=\hat{\hat{\eta}}\left(\theta \circ \hat{\tau}_{-t}\right)$. Therefore we have 


$$
\omega_{\hat{\hat{\eta}}\left(\theta^{\prime}\right), \eta_{\hat{h}}(x)} \circ \tau_{t}(z)=\omega_{\hat{\hat{\eta}}\left(\theta^{\prime} \circ \hat{\tau}_{t}\right), \eta_{\hat{h}}\left(\hat{\tau}_{-t}(x)\right)}(z), z \in M, t \in \mathbf{R}
$$

and hence the functional $\omega_{\hat{\hat{\eta}}\left(\theta^{\prime}, \eta_{\hat{h}}(x)\right.} \in M_{*}$ is entire analytic with respect to $\left\{\tau_{t}\right\}$. Furthermore, since $\left(\theta \circ \hat{\tau}_{-t}\right)(\hat{\pi}(\phi))=\theta\left(\hat{\pi}\left(\phi \circ \tau_{t}\right)\right)=\phi\left(\tau_{t}\left(\hat{\pi}_{*}(\theta)\right)\right)$ for $\theta \in \hat{M}_{*}$, if $\theta$ is entire analytic with respect to $\left\{\hat{\tau}_{t}\right\}$, then $\hat{\pi}_{*}(\theta)$ is entire analytic with respect to $\left\{\tau_{t}\right\}$. Thus

$$
\begin{aligned}
& \left(\hat{\hat{\pi}}(\theta) \hat{\hat{\eta}}\left(\theta^{\prime}\right) \mid \eta_{\hat{h}}(x)\right)=\theta\left(\hat{\pi}\left(\omega_{\eta_{\hat{h}}(x) \hat{\hat{\eta}}\left(\theta^{\prime}\right)}\right)^{*}\right) \\
& =\theta\left(\hat{\pi}\left(\omega_{\hat{\hat{\eta}}\left(\theta^{\prime}\right), \eta_{\hat{h}}(x)} \circ R \circ \tau_{-l / 2}\right)\right)=\left(R \circ \tau_{-t / 2} \circ \hat{\pi}_{*}(\theta) \hat{\hat{\eta}}\left(\theta^{\prime}\right) \mid \eta_{\hat{h}}(x)\right) .
\end{aligned}
$$

As the image of $\hat{\mathfrak{a}}_{0}$ by the GNS-mapping $\eta_{\hat{h}}$ as well as the image of entire analytic and $L^{2}$-bounded elements in $\hat{M}_{*}$ by the mapping $\hat{\hat{\eta}}$ are dense in $\mathfrak{S}$, we see that $\hat{\hat{\pi}}(\theta)=R \circ \tau_{-l / 2} \circ \hat{\pi}_{*}(\theta)$ for any entire analytic element $\theta \in \hat{M}_{*}$ for $\left\{\hat{\tau}_{t}\right\}$.

Finally we will show that $\hat{\hat{\pi}}\left(\theta^{\#}\right)=\hat{\hat{\pi}}(\theta)^{*}$. By Propositions 3.4 and 3.6 , if $\phi \in\left(M_{*}\right)_{\tau}$, we have

$$
\begin{aligned}
& \phi\left(\hat{\hat{\pi}}\left(\theta^{\#}\right)\right)=\phi\left(R \circ \tau_{-l / 2} \circ \hat{\pi}_{*}\left(\theta^{\#}\right)\right)=\left(\phi^{*}\right)^{\#}\left(\hat{\pi}_{*}\left(\phi^{\#}\right)\right)=\theta^{\#}\left(\hat{\pi}\left(\phi^{* \#}\right)\right) \\
& =\theta^{\#}\left(\hat{\pi}\left(\phi^{*}\right)^{*}\right)=\theta^{*}\left(\hat{R} \circ \hat{\tau}_{i / 2}\left(\hat{\pi}_{*}\left(\phi^{*}\right)\right)^{*}\right)=\overline{\theta \circ \hat{R} \circ \hat{\tau}_{t / 2}\left(\hat{\pi}\left(\phi^{*}\right)\right)} \\
& =\overline{\theta\left(\hat{\pi}\left(\phi^{*} \circ R \circ \tau_{-l / 2}\right)\right)}=\overline{\phi^{*} \circ R \circ \tau_{-l / 2}\left(\hat{\pi}_{*}(\theta)\right)} \overline{\phi^{*}(\hat{\hat{\pi}}(\theta))} \\
& =\phi\left(\hat{\hat{\pi}}(\theta)^{*}\right),
\end{aligned}
$$

and hence we obtain $\hat{\hat{\pi}}\left(\theta^{\#}\right)=\hat{\hat{\pi}}(\theta)^{*}$.

Proposition 3.16. The Haar weight $\hat{h}$ satisfies the strong left invariance.

Proof. Let $\theta$ be any element in $\hat{M}_{*}$. Let $x$ and $y$ be any elements in $\mathfrak{n}_{\hat{h}}$. If $\theta$ is of the form $\omega_{\xi, \eta}$ for $\xi=\eta_{\hat{h}}(a)$ and $\eta=\eta_{\hat{h}}(b)$ with $a, b \in \mathfrak{n}_{\hat{h}}$, then

$$
\begin{aligned}
(\theta \otimes \hat{h})((1 \otimes & \left.\left.\hat{\tau}_{t}(y)\right)^{*} \hat{\delta}\left(\hat{\tau}_{t}(x)\right)\right)=(\hat{h} \otimes \hat{h})\left(\left(b \otimes \hat{\tau}_{t}(y)\right)^{*} \hat{\delta}\left(\left(\hat{\tau}_{t}(x)\right)(a \otimes 1)\right)\right. \\
& =\left(\eta_{\hat{h} \otimes \hat{h}}\left(\hat{\delta}\left(\hat{\tau}_{t}(x)\right)(a \otimes 1)\right) \mid \eta_{\hat{h} \otimes \hat{h}}\left(b \otimes \hat{\tau}_{t}(y)\right)\right) \\
& =\left(\hat{W} \eta_{\hat{h} \otimes \hat{h}}\left(a \otimes \hat{\tau}_{t}(x)\right) \mid \eta_{\hat{h} \otimes \hat{h}}\left(b \otimes \hat{\tau}_{t}(y)\right)\right) \\
& =\left(\eta_{\hat{h}}\left(\hat{\tau}_{t}(x)\right) \mid \hat{\hat{\pi}}\left(\theta^{*}\right) \eta_{\hat{h}}\left(\hat{\tau}_{t}(y)\right)\right)
\end{aligned}
$$

and hence we obtain 


$$
\begin{aligned}
& (\theta \circ \hat{R} \otimes \hat{h})\left(\hat{\delta}\left(\hat{\tau}_{t}(y)\right)^{*}\left(1 \otimes \hat{\tau}_{t}(x)\right)\right)=\overline{\left(\theta^{*} \circ \hat{R} \otimes \hat{h}\right)\left(\left(1 \otimes \hat{\tau}_{t}(x)\right)^{*} \hat{\delta}\left(\hat{\tau}_{t}(y)\right)\right)} \\
& =\overline{\left(\eta_{\hat{h}}\left(\hat{\tau}_{t}(y)\right) \mid \hat{\hat{\pi}}(\theta \circ \hat{R}) \eta_{\hat{h}}\left(\hat{\tau}_{t}(x)\right)\right)} \\
& =\left(\hat{\hat{\pi}}(\theta \circ \hat{R}) \eta_{\hat{h}}\left(\hat{\tau}_{t}(x)\right) \mid \eta_{\hat{h}}\left(\hat{\tau}_{t}(y)\right)\right), t \in \mathbb{R} .
\end{aligned}
$$

In the above formulas (3.14) and (3.15) we may assume that $\theta$ is a finite linear combinations of $\omega_{\xi, \eta}$ 's. Since such elements form a dense subspace of $\hat{M}_{*}$, we may assume that $\theta$ is an arbitrary element in $\hat{M}_{*}$.

For any $x$ and $y$ in $\hat{\mathfrak{a}}_{0}$ we define two entire functions $F_{x, y, \theta}$ and $G_{x, y, \theta}$ by

$$
\begin{gathered}
F_{x, v, \theta}(\alpha)=\left(\eta_{\hat{h}}\left(\hat{\tau}_{\alpha}(x)\right) \mid \hat{\hat{\pi}}\left(\theta^{*}\right) \eta_{\hat{h}}\left(\hat{\tau}_{\bar{\alpha}}(y)\right)\right), \quad \alpha \in \mathbb{C} \\
G_{x, y, \theta}(\alpha)=\left(\hat{\hat{\pi}}(\theta \circ \hat{R}) \eta_{\hat{h}}\left(\hat{\tau}_{\alpha}(x)\right) \mid \eta_{\hat{h}}\left(\hat{\tau}_{\bar{\alpha}}(y)\right)\right), \quad \alpha \in \mathbb{C} .
\end{gathered}
$$

Of course, the function $F_{x, y, \theta}$ is bounded on $\mathbf{R}$ and $\mathbf{R}+(i / 2)$; hence it is bounded continuous function on $\mathbf{D}=\{\alpha \in \mathbf{C}: 0 \leq \operatorname{Im} \alpha \leq 1 / 2\}$. Similarly, the function $G_{x, y, \theta}$ is bounded continuous function on $\{\alpha \in \mathbf{C}:-1 / 2 \leq \operatorname{Im} \alpha \leq 0\}$. Since $\tau_{t}\left(\hat{\pi}_{*}\left(\theta^{\prime}\right)\right)=\hat{\pi}_{*}\left(\theta^{\prime} \circ \hat{\tau}_{-t}\right)$ and $R\left(\hat{\pi}_{*}\left(\theta^{\prime}\right)\right)=\hat{\pi}_{*}\left(\theta^{\prime} \circ \hat{R}\right)$ for any $\theta^{\prime} \in \hat{M}_{*}$, if $\theta$ is entire analytic with respect to $\left\{\hat{\tau}_{t}\right\}$, then for any $t \in \mathbb{R}$

$$
\begin{aligned}
F_{i, y, \theta}(t) & =\left(\hat{\pi}\left(\theta^{*}\right)^{*} \eta_{\hat{h}}\left(\hat{\tau}_{t}(x)\right) \mid \eta_{\hat{h}}\left(\hat{\tau}_{t}(y)\right)\right) \\
& =\left(\hat{\hat{\pi}}\left(\theta \circ \hat{R} \circ \hat{\tau}_{-t / 2}\right) \eta_{\hat{h}}\left(\hat{\tau}_{t}(x)\right) \mid \eta_{\hat{h}}\left(\hat{\tau}_{t}(y)\right)\right) \\
& =\left(\hat{\hat{\pi}}(\theta \circ \hat{R}) H^{1 / 2} \eta_{\hat{h}}\left(\hat{\tau}_{t}(x)\right) \mid H^{-1 / 2} \eta_{\hat{h}}\left(\hat{\tau}_{t}(y)\right)\right) \\
& =\left(\hat{\hat{\pi}}(\theta \circ \hat{R}) \eta_{\hat{h}}\left(\hat{\tau}_{t-(l / 2)}(x)\right) \mid \eta_{\hat{h}}\left(\hat{\tau}_{t+(t / 2)}(y)\right)\right) \\
& =G_{x, y, \theta}(t-(i / 2)) .
\end{aligned}
$$

By the unicity theorem we find that $G_{x, y, \theta}(\alpha)=f_{x, y, \theta}(\alpha+(i / 2)), \alpha \in \mathbf{C}$ for any $\left\{\hat{\tau}_{t}\right\}$-entire analytic elements $\theta \in \hat{M}_{*}$. Thus the strong left invariance holds for $x, y \in \hat{\mathfrak{a}}_{0}$ by (3.14) and (3.15).

Next, we consider the case where $x \in \mathfrak{n}_{\hat{h}}$ and $y \in \hat{\mathfrak{a}}_{0}$. Let $\left\{x_{n}\right\}$ be a sequence in $\hat{\mathfrak{a}}_{0}$ such that $\eta_{\hat{h}}\left(x_{n}\right) \rightarrow \eta_{\hat{h}}(x)$. Denote the entire functions $F_{x_{n}, v, \theta}$ by $F_{n}$, which are entire analytic even if $x$ is not entire analytic with respect to $\left\{\hat{\tau}_{t}\right\}$, since $\theta$ is entire analytic with respect to $\left\{\hat{\tau}_{t}\right\}$. Then the sequence $\left\{F_{n}\right\}$ converges uniformly on $\mathbb{R}$ and $\mathbf{R}+(i / 2)$ to functions (3.16) and (3.17), respectively. Thus the Phragmen-Lindelöf theorem tells us that $\left\{F_{n}\right\}$ converges to a bounded continuous function $F$ on $\mathbf{D}=\{\alpha \in \mathbf{C}: 0 \leq \operatorname{Im} \alpha \leq 1 / 2\}$ which is analytic in $\mathbf{D}$ and satisfies the strong left invariance:

$$
\begin{gathered}
F(t)=(\theta \otimes \hat{h})\left(\left(1 \otimes \hat{\tau}_{t}(y)\right)^{*} \hat{\delta}\left(\hat{\tau}_{t}(x)\right)\right) \\
F(t+(i / 2))=(\theta \circ \hat{R} \otimes \hat{h})\left(\hat{\delta}\left(\hat{\tau}_{t}(y)\right)^{*}\left(1 \otimes \hat{\tau}_{t}(x)\right)\right) .
\end{gathered}
$$


Furthermore, the case where $x$ and $y$ are arbitrary elements in $\mathfrak{n}_{\hat{h}}$ is treated similarly by approximating $y$ by elements in $\hat{\mathfrak{a}}_{0}$ in addition to the above argument.

QED

Summarizing the above propositions, we have the following theorem.

Theorem 3.17. The family $(\hat{M}, \hat{\delta}, \hat{R}, \hat{\tau}, \hat{h})$ is a Woronowicz algebra and $\hat{W}$ is the Kac-Takesaki operator.

The family $(\hat{M}, \hat{\delta}, \hat{R}, \hat{\tau}, \hat{h})$ is called the dual Woronowicz algebra for the Woronowicz algebra $(M, \delta, R, \tau, h)$. A Haar weight on the dual Woronowicz algebra is sometimes called the Plancherel weight for $(M, \delta, R, \tau, h)$.

Remark 3.18. A Woronowicz algebra is said to compact (resp. discrete), if the Haar weight (resp. Plancherel weight) is bounded. If a Woronowicz algebra is compact, then the dual Woronowicz algebra has the counit $\hat{\varepsilon}: \hat{\pi}(\varphi) \rightarrow \varphi(1)$ as in Remark 3.19 below. If a Woronowicz algebra $(M, \delta, R, \tau, h)$ is compact and discrete, then it is automatically finite dimensional by a similar discussion as in [23]. Hence the modular automorphism $\left\{\sigma_{t}^{h}\right\}$ is inner, i.e. there exists an invertible positive operator $d$ in the fixed point subalgebra $M^{\sigma^{h}}$ with respect to $\left\{\sigma_{t}^{h}\right\}$ such that $\sigma_{t}^{h}(x)=d^{t t} x d^{-t t}$ for $x \in M$ and $t \in \mathbf{C}$. Therefore the mapping: $x \in M \rightarrow h\left(d^{-1} x\right) \in \mathbf{C}$ is a faithful normal trace on $M$, which we denote by $t r$. On the other hand, the Radon-Nikodym derivatives in this case satisfy $\rho=1$ and $\hat{\rho}=1$ by Remark 1.3 , and so $\Delta=H=\hat{\Delta}$ by Lemma 2.14 . Since $\rho=1$ and $R(\rho)=\rho^{-1}$, we see that $\operatorname{tr} \circ R(x)=\operatorname{tr}\left(d^{2} x\right)$ for $x \in M$. Since $\operatorname{tr} \circ R$ is also a faithful normal trace on $M$, the Radon-Nikodym derivative $d^{2}$ of $t r \circ R$ with respect to $\operatorname{tr}$ belongs to the center of $M$. Hence the modular automorphism $\left\{\sigma_{t}^{h}\right\}$ turns out to be trivial, i.e. $\Delta=1$, and hence $H=\hat{\Delta}=1$. Therefore the deformation automorphisms in the Woronowicz algebra $(M, \delta, R, \tau, h)$ and its dual are trivial. Consequently, $(M, \delta, R, t r)$ is a Kac algebra. In other words, the Woronowicz algebra $(M, \delta, R, \tau, h)$ and its dual are Kac algebras.

Remark 3.19. If the Haar weight is bounded, then it is $L^{2}$-bounded and satisfies $W(\xi \otimes \hat{\eta}(h))=\xi \otimes \hat{\eta}(h)$ for all $\xi \in \mathfrak{S}$ as shown in [1]. Indeed, for any $L^{2}$-bounded $\varphi \in M_{*}$ and $x, y \in \mathfrak{n}_{h}$ we find

$$
\begin{gathered}
\left(\hat{\eta}(\varphi) \otimes \hat{\eta}(h) \mid W \eta_{h \otimes h}(x \otimes y)\right)=(\varphi \otimes h)\left(\left(x^{*} \otimes 1\right) \delta\left(y^{*}\right)\right) \\
=\varphi\left(x^{*}\right) h\left(y^{*}\right)=\left(\hat{\eta}(\varphi) \otimes \hat{\eta}(h) \mid \eta_{h \otimes h}(x \otimes y)\right) .
\end{gathered}
$$

By the uniqueness of a bounded Haar weight up to scalar shown as in Remark 1.2 , the subspace $\{\eta \in \mathfrak{S}: W(\xi \otimes \eta)=\xi \otimes \eta, \xi \in \mathfrak{S}\}$ is one dimensional. The state 
$\omega_{\eta}$ for a normalized vector $\eta$ in this subspace gives us a normalized Haar weight $h$ on $M$ as well as the counit $\hat{\varepsilon}$ on $\hat{M}$ :

$$
\left(\mathrm{id} \otimes \omega_{\eta}\right)(\delta(x))=\omega_{\eta}(x) 1 \text { for } x \in M
$$

and

$$
\omega_{\eta}(\hat{\pi}(\varphi))=\omega_{\hat{\eta}(h)}(\hat{\pi}(\varphi))=\varphi(1) \text { for } \varphi \in M_{*} .
$$

Therefore we obtain $\hat{\pi}_{*}(\hat{\varepsilon})=1$ for $\hat{\varepsilon}=\left.\omega_{\eta}\right|_{\hat{M}}$, and $(\hat{\varepsilon} \otimes \mathrm{id}) \circ \hat{\delta}=\mathrm{id}=(\mathrm{id} \otimes \hat{\varepsilon}) \circ \hat{\delta}$.

\section{§4. Duality Theorem}

Let $(M, \delta, R, \tau, h)$ be a Woronowicz algebra and $(\hat{M}, \hat{\delta}, \hat{R}, \hat{\tau}, \hat{h})$ its dual Woronowicz algebra:

$$
\hat{\delta}(x)=\hat{W}(1 \otimes x) \hat{W}^{*}, \quad \hat{R}(x)=J x^{*} J, \quad \hat{\tau}_{t}(x)=H^{t t} x H^{-i t}
$$

and

$$
\hat{h}\left(\hat{\pi}_{\ell}(\xi)^{*} \hat{\pi}_{\ell}(\xi)\right)=\left\{\begin{array}{cc}
(\xi \mid \xi) & \text { if } \quad \xi \in \hat{\mathfrak{U}}^{\prime \prime} \\
\infty & \text { otherwise }
\end{array}\right.
$$

Then $\hat{W}=\sigma\left(W^{*}\right)$ is the Kac-Takesaki operator for the dual Woronowicz algebra. The bidual Woronowicz algebra $(\hat{\hat{M}}, \hat{\hat{\delta}}, \hat{\hat{R}}, \hat{\hat{\tau}}, \hat{\hat{h}})$ is defined to be the dual Woronowicz algebra of $(\hat{M}, \hat{\delta}, \hat{R}, \hat{\tau}, \hat{h})$ constructed in the same manner as in Section 3. The Kac-Takesaki operator $\hat{\hat{W}}$ is then given by a unitary $\sigma\left(\hat{W}^{*}\right)$. Thus $\hat{\hat{W}}=W$. If $\hat{\hat{M}}$ is shown to be a subalgebra of $M$, then $\hat{\hat{\delta}}, \hat{\hat{R}}, \hat{\hat{\tau}}$ and $\hat{\hat{h}}$ agree with $\delta, R, \tau$ and $h$ on $\hat{\hat{M}}$.

Our main assertion is the following duality theorem for a Woronowicz algebra.

Theorem 4.1. A Woronowicz algebra is isomorphic to the bidual Woronowicz algebra:

$$
(M, \delta, R, \tau, h) \cong(\hat{\hat{M}}, \hat{\hat{\delta}}, \hat{\hat{R}}, \hat{\hat{\tau}}, \hat{\hat{h}})
$$

We have already used the notation $\hat{\hat{\mathfrak{A}}}, \hat{\hat{S}}, \hat{\hat{\eta}}$ and $\hat{\hat{\pi}}$ for the bidual Woronowicz algebra corresponding to those for the dual Woronowicz algebra, i.e. $(\hat{\hat{\mathfrak{A}}}, \hat{\hat{S}})$ is a pair of the left Hilbert algebra and the left involution operator corresponding to $(\hat{\mathfrak{A}}, \hat{S}) ; \hat{\hat{\eta}}$ is the linear mapping of the set of $L^{2}$-bounded elements in $\hat{M}_{*}$ to corresponding to $\hat{\eta}$; and $\hat{\hat{\pi}}$ is the Fourier transform from $\hat{M}_{*}$ to $\hat{\hat{M}}$ corresponding to $\hat{\pi}$. 
Lemma 4.2. The left Hilbert algebra $\hat{\hat{\mathfrak{U}}}$ is a subalgebra of the achieved left Hilbert algebra $\mathfrak{A}_{h}$ and $\hat{\hat{S}} \subset S$.

Proof. Let $\hat{\hat{\eta}}(\theta)$ be any element in $\hat{\hat{\mathfrak{U}}}=\hat{\hat{\eta}}(\hat{\mathfrak{b}})$. For any $\xi^{\prime}, \eta^{\prime}$ in the right Hilbert algebra $\mathfrak{A}^{\prime}$ associated with $\mathfrak{U}_{h}$, we denote by $\phi$ the restriction of $\omega_{\xi^{\prime}, \eta^{\prime}}$ to $M$. Since $\hat{\eta}(\phi)=\xi^{\prime} \eta^{\prime b}$, we have

$$
\begin{aligned}
& \left(\pi_{r}\left(\eta^{\prime}\right) \hat{\hat{\eta}}(\theta) \mid \xi^{\prime}\right)=\left(\hat{\hat{\eta}}(\theta) \mid \xi^{\prime} \eta^{\prime b}\right)=(\hat{\hat{\eta}}(\theta) \mid \hat{\eta}(\phi)) \\
& =\left(\hat{\hat{\eta}}(\theta) \mid \eta_{\hat{h}}(\hat{\pi}(\phi))\right)=\theta\left(\hat{\pi}(\phi)^{*}\right)=\theta\left(\hat{\pi}\left(\phi^{\#}\right)\right) \\
& =\phi^{\#}\left(\hat{\pi}_{*}(\theta)\right)=\phi^{*}\left(R \circ \tau_{-t / 2} \circ \hat{\pi}_{*}(\theta)\right)=\left(\hat{\hat{\pi}}(\theta) \eta^{\prime} \mid \xi^{\prime}\right),
\end{aligned}
$$

where $\pi_{r}$ is the representation of the right Hilbert algebra $\mathfrak{A}^{\prime}$. Thus $\pi_{r}\left(\eta^{\prime}\right) \hat{\hat{\eta}}(\theta)=\hat{\hat{\pi}}(\theta) \eta^{\prime}$ for all $\eta^{\prime} \in \mathfrak{U}^{\prime}$. Hence $\hat{\hat{\eta}}(\theta)$ is left bounded. On the other hand,

$$
\begin{aligned}
& \left(\hat{\hat{S}} \hat{\hat{\eta}}(\theta) \mid \xi^{\prime} \eta^{\prime b}\right)=\left(\hat{\hat{\eta}}\left(\theta^{\#}\right) \mid \hat{\eta}(\phi)\right)=\left(\hat{\hat{\pi}}\left(\theta^{\#}\right) \eta^{\prime} \mid \xi^{\prime}\right) \\
& =\left(\hat{\hat{\pi}}(\theta)^{*} \eta^{\prime} \mid \xi^{\prime}\right)=\left(\eta^{\prime} \xi^{\prime} b \mid \hat{\hat{\eta}}(\theta)\right)=\left(F\left(\xi^{\prime} \eta^{\prime b}\right) \mid \hat{\hat{\eta}}(\theta)\right) .
\end{aligned}
$$

Therefore $\hat{\hat{\eta}}(\theta)$ belongs to the domain of operator $S$ and $\hat{\hat{S}}=S$ on $\hat{\hat{\mathfrak{A}}}$. Hence $\hat{\hat{\eta}}(\theta) \in \mathfrak{U}_{h}$. Thus $\hat{\hat{\mathfrak{U}}}$ is identified with a vector subspace of $\mathfrak{U}_{h}$ together with their left involution operators.

To show the coincidence of multiplication structures, it remains to show that $\pi_{\ell}(\hat{\hat{\eta}}(\theta))=\hat{\hat{\pi}}(\theta)$, where $\pi_{\ell}$ is the representation of the left Hilbert algebra $\mathfrak{A}_{h}$. Let $\xi^{\prime}$ and $\eta^{\prime}$ be any elements in $\mathfrak{A}^{\prime}$. Then we have

$$
\left(\pi_{\ell}(\hat{\hat{\eta}}(\theta)) \xi^{\prime} \mid \eta^{\prime}\right)=\left(\pi_{r}\left(\xi^{\prime}\right) \hat{\hat{\eta}}(\theta) \mid \eta^{\prime}\right)=\left(\hat{\hat{\pi}}(\theta) \xi^{\prime} \mid \eta^{\prime}\right)
$$

by (4.1).

QED

Denote by $W$ the Woronowicz algebra $(M, \delta, R, \tau, h)$. We will consider the co-opposite Woronowicz algebra $W^{\circ}$ and the commutant Woronowicz algebra $W^{\prime}$ by using the mappings: $x \rightarrow R(x)=\hat{J} x^{*} \hat{J}$ and $x \rightarrow J x^{*} J$, respectively.

Proposition 4.3. (i) If $\delta^{\circ}=\sigma \circ \delta, R^{\circ}=R, \tau_{t}^{\circ}=\tau_{-t}$, and $h^{\circ}=h \circ R$, then $W^{\circ}=\left(M, \delta^{\circ}, R^{\circ}, \tau^{\circ}, h^{\circ}\right)$ is a Woronowicz algebra and the Kac-Takesaki operator $W^{\circ}$ is of the form $V W$, where $V \in \mathscr{L}(\mathfrak{S} \otimes \mathfrak{S})$ is the flip operator: $\xi \otimes \eta \rightarrow \eta \otimes \xi$.

(ii) If $\delta^{\prime}=\operatorname{Ad}_{J \otimes J} \circ \delta \circ \mathrm{Ad}_{J}, R^{\prime}=\operatorname{Ad}_{J} \circ R \circ \operatorname{Ad}_{J}, \tau_{t}^{\prime}=\operatorname{Ad}_{J} \circ \tau_{-t} \circ \operatorname{Ad}_{J}$ and $h^{\prime}(x)=h(J x J)$ for $x \in M_{+}^{\prime}$, then $W^{\prime}=\left(M^{\prime}, \delta^{\prime}, R^{\prime}, \tau^{\prime}, h^{\prime}\right)$ is a Woronowicz algebra 
and the Kac-Takesaki operator $W^{\prime}$ is of the form $\operatorname{Ad}_{J \otimes J}(W)=\operatorname{Ad}_{U \otimes 1}\left(W^{*}\right)$, where $U=J \hat{J}$.

Proof. (i) It is easy to see that $\delta^{\circ}$ is coproduct, $R^{\circ}$ is a unitary antipode, $\left\{\tau_{t}^{\circ}\right\}$ is a deformation automorphism and $h^{\circ}$ is a $\left\{\tau_{t}^{\circ}\right\}$-invariant faithful semifinite normal weight on $M$ such that $h^{\circ}$ is left invariant and commutes with the weight $h^{\circ} \circ R^{\circ}$. It remains to show that the weight $h^{\circ}$ satisfies the strong left invariance. If $x, y \in \mathfrak{a}_{h} \cap \mathfrak{a}_{h \circ R}$ and $\phi \in\left(M_{*}\right)_{\tau}$ then, by the strong left invariance of the Woronowicz algebra $W$, there exists a bounded continuous function $F_{x, y, \phi}$ on the strip $\mathbf{D}=\{z \in \mathbf{C}: 0 \leq \operatorname{Im} z \leq 1 / 2\}$ such that $F_{x, y, \phi}$ is analytic in $\mathbf{D}$ and satisfies the boundary condition:

$$
\begin{gathered}
F_{x, y, \phi}(t)=\left(\phi \circ \tau_{t} \otimes h\right)\left((1 \otimes y)^{*} \delta(x)\right) \\
F_{x, y, \phi}(t+(i / 2))=\left(\phi \circ R \circ \tau_{t} \otimes h\right)\left(\delta(y)^{*}(1 \otimes x)\right) .
\end{gathered}
$$

Let $F_{R(y)^{\dagger}, R(x)^{\dagger}, \phi}(z)=F_{x, y, \phi}(-z+(i / 2))$. Then $F_{R(y)^{\dagger}, R(x)^{r}, \phi}$ is a bounded continuous function on $\mathbf{D}$ such that it is analytic in $\mathbf{D}$ and satisfies the boundary condition:

$$
\begin{gathered}
F_{R(y)^{+}, R(x)^{\dagger}, \phi}(t)=\left(\phi \circ \tau_{t}^{\circ} \otimes h^{\circ}\right)\left((1 \otimes R(x)) \delta^{\circ}\left(R\left(y^{*}\right)\right)\right) \\
F_{R(y)^{*}, R(x)^{*}, \phi}(t+(i / 2))=\left(\phi \circ R^{\circ} \circ \tau_{t}^{\circ} \otimes h^{\circ}\right)\left(\delta^{\circ}(R(x))\left(1 \otimes R\left(y^{*}\right)\right)\right) .
\end{gathered}
$$

Here notice that $R\left(\mathfrak{a}_{h} \cap \mathfrak{a}_{h \circ R}\right)=\mathfrak{a}_{h} \cap \mathfrak{a}_{h \circ R}$. For any $x, y$ in $\mathfrak{n}_{h^{\circ}}$ there exist sequences $\left\{x_{n}\right\}$ and $\left\{y_{n}\right\}$ in $\mathfrak{a}_{h} \cap \mathfrak{a}_{h \circ R}$ such that $\eta_{h^{\circ}}\left(x_{n}\right) \rightarrow \eta_{h^{\circ}}(x)$ and $\eta_{h^{\circ}}\left(y_{n}\right) \rightarrow \eta_{h^{\circ}}(y)$. The sequence $\left\{F_{R\left(y_{n}\right)^{*}, R\left(x_{n}\right)^{\prime}, \phi}\right\}$ is Cauchy on $\mathbf{R}$ and $\mathbf{R}+(i / 2)$ in the uniform topology. Hence, by the Phragmen-Lindelöf Theorem, there exists a function $F$ on D such that $F_{R\left(y_{n}\right)^{*}, R\left(x_{n}\right)^{*}, \phi}$ converges uniformly to $F$. Thus $F$ is a bounded continuous function on $\mathbf{D}$ such that it is analytic in $\mathbf{D}$ and satisfies the same boundary condition (4.2).

(ii) The proof is accomplished in the same manner as in (i).

QED

The entire analytic part $\left(M_{*}\right)_{\tau^{\circ}}$ of the predual is the same as $\left(M_{*}\right)_{\tau}$ as a set. The product $\phi, \psi \rightarrow(\phi \otimes \psi) \circ \delta^{\circ}$ in the former coincides with $\psi * \phi$ in the latter. Since the entire analytic part $\mathfrak{a}_{0}$ for $W$ coincides with that for $W^{\circ}$, the involutive algebra $\mathfrak{b}^{\circ}$ for $W^{\circ}$ corresponding to $\mathfrak{b}$ in Definition 2.3 is the same as $\mathfrak{b}$ as a set. Therefore the involutive algebra $\hat{\mathfrak{A}}^{\circ}=\hat{\eta}^{\circ}\left(\mathfrak{b}^{\circ}\right)$ is $*$-antiisomorphic to $\hat{\mathfrak{U}}=\hat{\eta}(\mathfrak{b})$.

By means of the correspondence: $\eta_{h^{\circ}}(x) \rightarrow J \rho^{-1 / 2} J \eta_{h}(x)=\eta_{h}\left(L_{l / 2} x\right)$ for $x \in \mathfrak{a}_{0}$, the standard representation Hilbert space for $\left(M^{\circ}, h^{\circ}\right)$ is identified with the Hilbert space $\mathfrak{S}$ for $(M, h)$. Similarly, by the correspondence: $\eta_{h^{\prime}}(x) \rightarrow$ $J \eta_{h}(J x J)$ the standard representation Hilbert space for $\left(M^{\prime}, h^{\prime}\right)$ is identified with the Hilbert space $\mathfrak{S}$ for $(M, h)$. The left Hilbert algebra $\mathfrak{A}_{h^{\prime}}$ is then identified with the right Hilbert algebra $\mathfrak{A}^{\prime}$ associated with $\mathfrak{A}_{h}$. Hence

$$
\pi_{\rho}^{\prime}\left(\eta_{h^{\prime}}(x)\right)=J \pi_{\ell}\left(\eta_{h}(J x J)\right) J=\pi_{r}\left(\eta_{h}\left(J x^{*} J\right)\right), x \in \mathfrak{a}_{h^{\prime}} .
$$


Indeed,

$$
\begin{aligned}
& \pi_{\ell}^{\prime}\left(\eta_{h^{\prime}}(x)\right) \eta_{h^{\prime}}(y)=\eta_{h^{\prime}}(x y)=J \eta_{h}(J x y J) \\
& =J \pi_{\ell}\left(\eta_{h}(J x J)\right) \eta_{h}(J y J)=J \pi_{\ell}\left(\eta_{h}(J x J)\right) J \eta_{h^{\prime}}(y) .
\end{aligned}
$$

Let $\left\{J^{\circ}, \Delta^{\circ}, \rho^{\circ}, H^{\circ}\right\}$ and $\left\{J^{\prime}, \Delta^{\prime}, \rho^{\prime}, H^{\prime}\right\}$ be families of operators which appear in $W^{\circ}$ and $W^{\prime}$ respectively, corresponding to the family $\{J, \Delta, \rho, H\}$ for $W$. Then

$$
J^{\circ}=J, \quad \Delta^{\circ}=\Delta_{h \circ R}, \quad \rho^{\circ}=\rho^{-1}, \quad H^{\circ}=H^{-1}
$$

and

$$
J^{\prime}=J, \quad \Delta^{\prime}=\Delta^{-1}, \quad \rho^{\prime}=J \rho J, \quad H^{\prime}=H^{-1} .
$$

If $x, y \in \mathfrak{n}_{h}$, then $\rho^{i t} x, \rho^{-i t} y \in \mathfrak{n}_{h}$ and hence

$$
\rho^{t t} \omega_{x, y}=\omega_{\rho^{u t} x, y}=\omega_{\left(L_{-t} x^{*}\right)^{*}, y} \text { and } \omega_{x, y} \rho^{t t}=\omega_{x, \rho^{-t} y}=\omega_{x,\left(L_{t} y^{*}\right)^{+}} .
$$

Since $\delta\left(\rho^{i t}\right)=\rho^{i t} \otimes \rho^{i t} \quad$ implies $\quad \rho^{i t}(\phi * \psi)=\left(\rho^{i t} \phi\right) *\left(\rho^{i t} \psi\right) \quad$ and $\quad(\phi * \psi) \rho^{i t}=$ $\left(\phi \rho^{t t}\right) *\left(\psi \rho^{t t}\right)$ for $\phi, \psi \in M_{*}$, if $\phi, \psi \in \mathfrak{b}$, then these functions in $t$ can be extended to the whole complex plane by their analytic continuation. We sometimes use the formal notation such as $\phi \rho^{ \pm 1 / 2}$ or $\phi \rho^{ \pm 1}$ for them.

Proposition 4.4 Let $W^{\wedge}$ denote the dual Woronowicz algebra of $W$.

(i) $\left(W^{\circ}\right)^{\wedge}=\left(W^{\wedge}\right)^{\prime}$.

(ii) $\left(W^{\prime}\right)^{\wedge}=\left(W^{\wedge}\right)^{\circ}$.

Proof. (i) Let $\hat{\mathfrak{a}}^{\circ}=\hat{\eta}^{\circ}\left(\mathfrak{b}^{\circ}\right)$ be a left Hilbert algebra constructed from $W^{\circ}$ in the same way as in Definition 2.3. Let $\hat{\pi}^{\circ}$ and $\hat{\eta}^{\circ}$ denote the mappings corresponding to $\hat{\pi}$ and $\hat{\eta}$. Then for any $\phi$ in $\mathfrak{b}$, we have

$$
\hat{\eta}^{\circ}(\phi)=J \rho^{1 / 2} J \hat{\eta}(\phi)=\hat{\eta}\left(\phi \rho^{1 / 2}\right), \quad \hat{S}^{\circ} \hat{\eta}^{\circ}(\phi)=\hat{F} \hat{\eta}\left(\phi \rho^{1 / 2}\right)
$$

and

$$
\hat{\pi}^{\circ}(\phi)=\pi_{r}\left(\hat{\eta}\left(\phi \rho^{1 / 2}\right)\right),
$$

where $\hat{S}^{\circ}$ is the left involution operator for $\mathfrak{U}_{h^{\circ}}$ and $\pi_{r}$ is the representation of the right Hilbert algebra associated with $\hat{\mathfrak{U}}$. Indeed, $\hat{F} \hat{\eta}(\psi)=\hat{\eta}\left(\left(\rho^{-1} \psi^{*}\right) \circ R \circ \tau_{\iota / 2}\right)$ for $\psi$ in $\mathfrak{b}$ by Lemma 2.15 and hence

$$
\begin{aligned}
\hat{F} \hat{\eta}\left(\phi \rho^{1 / 2}\right) & =\hat{\eta}\left(\left(\rho^{-1 / 2} \phi^{*}\right) \circ R \circ \tau_{t / 2}\right)=\hat{\eta}\left(\left(\phi^{*} \circ R \circ \tau_{t / 2}\right) \rho^{1 / 2}\right) \\
& =\hat{\eta}^{\circ}\left(\phi^{*} \circ R \circ \tau_{t / 2}\right)=\hat{\eta}^{\circ}\left(\phi^{*} \circ R \circ \tau_{-t / 2}^{\circ}\right)=\hat{S}^{\circ} \hat{\eta}^{\circ}(\phi)
\end{aligned}
$$

for $\phi$ in $\mathfrak{b}$. By means of this identification, it is straightforward to verify that assertion (i) holds. 
(ii) The predual $\left(M^{\prime}\right)_{*}$ of $M^{\prime}$ coincides with the set $\left\{\phi^{\prime} \in\left(M^{\prime}\right)_{*}: \phi^{\prime}(x)=\right.$ $\left.\phi\left(J x^{*} J\right), \phi \in M_{*}\right\}$. Since, for $x \in \mathfrak{a}_{h^{\prime}}$ and an $L^{2}$-bounded $\phi^{\prime} \in\left(M^{\prime}\right)_{*}$ with $\phi^{\prime}(x)=$ $\phi\left(J x^{*} J\right)$,

$$
\begin{aligned}
\left(\hat{\eta}^{\prime}\left(\phi^{\prime}\right) \mid \eta_{h^{\prime}}(x)\right) & =\phi^{\prime}\left(x^{*}\right)=\phi(J x J)=\left(\hat{\eta}(\phi) \mid \eta_{h}\left(J x^{*} J\right)\right) \\
& =\left(\eta_{h^{\prime}}\left(x^{*}\right) \mid J \hat{\eta}(\phi)\right)=\left(\hat{\eta}(\phi) \mid \Delta^{-1 / 2} \eta_{h^{\prime}}(x)\right),
\end{aligned}
$$

the vector $\hat{\eta}(\phi)$ belongs to the domain of $\Delta^{-1 / 2}$ and $\hat{\eta}^{\prime}\left(\phi^{\prime}\right)=\Delta^{-1 / 2} \hat{\eta}(\phi)$. For any $\phi, \psi \in \mathfrak{b}$ we see that

$$
\hat{\pi}^{\circ}(\phi) \hat{\eta}^{\circ}(\psi)=\hat{\eta}^{\circ}(\phi * \psi)=\hat{\eta}\left((\phi * \psi) \rho^{1 / 2}\right)=\Delta^{1 / 2} \hat{\eta}^{\prime}\left(\left(\phi \rho^{1 / 2} * \psi \rho^{1 / 2}\right)^{\prime}\right) .
$$

It is straightforward to verify that $(\phi \otimes \psi)^{\prime}=\phi^{\prime} \otimes \psi^{\prime}$. Hence

$$
\hat{\pi}^{\circ}(\phi) \hat{\eta}^{\circ}(\psi)=\Delta^{1 / 2} \hat{\eta}^{\prime}\left(\left(\phi \rho^{1 / 2}\right)^{\prime} *\left(\psi \rho^{1 / 2}\right)^{\prime}\right)=\left(\Delta^{1 / 2} \hat{\pi}^{\prime}\left(\left(\phi \rho^{1 / 2}\right)^{\prime}\right) \Delta^{-1 / 2}\right) \hat{\eta}^{\circ}(\psi) .
$$

Hence $\hat{\pi}^{\prime}\left(\left(\phi \rho^{1 / 2}\right)^{\prime}\right)=\Delta^{-1 / 2} \hat{\pi}^{\circ}(\phi) \Delta^{1 / 2}$ for $\phi \in M_{*}$. Since $\hat{\pi}^{\circ}(\phi)=\hat{R}(\hat{\pi}(\phi))=\hat{\pi}(\phi \circ R)$, we have

$$
\Delta^{-1 / 2} \hat{\pi}^{\circ}(\phi) \Delta^{1 / 2}=\Delta^{-1 / 2} \hat{\pi}(\phi \circ R) \Delta^{1 / 2}=\hat{\pi}\left(\phi \circ R \circ \tau_{-l / 2}\right) .
$$

Using these fact in mind, we get the proof of $\left(W^{\prime}\right)^{\wedge}=\left(W^{\wedge}\right)^{\circ}$.

Corollary 4.4.1. (i) Let $\{\hat{J}, \hat{\Delta}, \hat{\rho}, H\}$ be the family of operators which appear in $W^{\wedge}$. Then $\left\{\hat{J}, \hat{\Delta}^{-1}, \hat{J} \hat{\rho} \hat{J}, H^{-1}\right\}$ is the family of operators for $\left(W^{\circ}\right)^{\wedge}=\left(W^{\wedge}\right)^{\prime}$ and $\left\{\hat{J}, \hat{\Delta}_{\hat{h} \circ \hat{R}}, \hat{\rho}^{-1}, H^{-1}\right\}$ for $\left(W^{\prime}\right)^{\wedge}=\left(W^{\wedge}\right)^{\circ}$.

(ii) Let $\Delta^{\circ \wedge}$ and $\Delta^{\text {s }}$ be the modular operators for $W^{\circ}$ and $W^{\prime}$ corresponding to $\hat{\Delta}$ for $W$. Then $\Delta^{\circ \wedge}$ is the closure of $J \rho^{-1} J H^{-1}$ and $\Delta^{\wedge \wedge}$ is the closure of $\rho H^{-1}$.

Proof. It is clear from (4.3) and (4.4).

Proof of Theorem 4.1. By virtue of Lemma 4.2, $\hat{\hat{S}} \subset S$. If $\hat{\hat{\Delta}}$ commute with $\Delta$, then $\hat{\hat{S}}=S$. Hence $\mathfrak{U}_{h}$ is the achieved left Hilbert algebra associated with $\hat{\hat{\mathfrak{U}}}$. It remains to show the commutativity of the modular operators $\Delta$ and $\hat{\hat{\Delta}}$. By Corollaries 2.14.1 and 3.6.1, $\operatorname{Ad}_{\hat{\hat{\Delta}}^{\prime \prime}}=\operatorname{Ad}_{H^{\prime \prime}}$ and $\operatorname{Ad}_{\Delta^{\prime \prime}}=\operatorname{Ad}_{H^{\prime \prime}}$ on $\hat{M}$, which prove $\operatorname{Ad}_{\hat{\hat{\Delta}}^{t \prime}}=\operatorname{Ad}_{\Delta^{\prime \prime}}$ on $\hat{M}$. Recall that $\hat{\hat{\Delta}}$ commutes with $\hat{\rho}$ by Lemma 2.14. Since $\hat{\rho}^{i s} \in \hat{M}$ for $s \in \mathbb{R}$, it follows that $\operatorname{Ad}_{\Delta^{u}}\left(\hat{\rho}^{i s}\right)=\hat{\rho}^{\prime s}$. Hence $\Delta$ commutes with $\hat{\rho}$.

We repeat the same argument for the co-opposite Woronowicz algebra $W^{\circ}$. Then we find that $\Delta^{\circ}=\Delta_{h \circ R}$ commutes with $\hat{J} \hat{\rho} \hat{J}$ by Corollary 4.4.1.

Since $\sigma_{t}^{\hat{h}}\left(\hat{\rho}^{t s}\right)=\hat{\rho}^{t s}$ for $t, s \in \mathbb{R}, \hat{\rho}^{t s}$ commutes with $\hat{\Delta}^{t t}=J \rho^{-t t} J H^{t t}$ as well as $J \rho^{i t} J$. The equality $\hat{\rho}^{i s}=\hat{R}\left(\hat{\rho}^{-t s}\right)=J \hat{\rho}^{i s} J$ implies that $\hat{\rho}^{t s}$ commutes with $\rho^{t t}$ and $J \rho^{\prime \prime} J$. Thus $\hat{\rho}$ commutes with $\rho$ and $J \rho J$. Hence $\hat{J} \hat{\rho} \hat{J}$ commutes with $\hat{J} \rho \hat{J}=\rho^{-1}$ 
and $\hat{J} J \rho J \hat{J}$. Since $\hat{\Delta}^{i t}=\hat{J} \hat{\Delta}^{t t} \hat{J}=\hat{J} J \rho^{-t t} J \hat{J} H^{t t}$, it follows that $J \rho^{t t} J=\hat{J} J \rho^{t t} J \hat{J}$ and hence $\hat{J} J \rho J \hat{J}=J \rho^{-1} J$. Thus $\hat{J} \hat{\rho} \hat{J}$ commutes with $\rho J \rho^{-1} J$. On the other hand, $\Delta^{t}=\rho^{t t} J \rho^{t} J \Delta_{h \circ R}^{t t}$ and $\Delta_{h \circ R}=\Delta^{\circ}$ by (4.3). Therefore the modular operator $\Delta$ is given by the closure of $\rho J \rho^{-1} J \Delta^{\circ}$. Hence $\Delta$ commutes with $\hat{J} \hat{\rho} \hat{J}$ and $H$ as well. Since $\hat{\hat{\Delta}}$ is the closure of $\hat{J} \hat{\rho} \hat{J} H$ by Lemma $2.14, \Delta$ commutes with $\hat{\hat{\Delta}}$. QED

Remark 4.5. When a Woronowicz algebra is commutative, the modular operator $\Delta$ is trivial, i.e., $\Delta=1$, and hence $H=\hat{\rho}$ by Lemma 2.14. since $\hat{J} \hat{\rho} \hat{J}=H^{-1}, H$ is affiliated with the center of $\hat{M}$ and so the deformation automorphism $\left\{\hat{\tau}_{t}\right\}$ is trivial on $\hat{M}$. By virtue of the duality, the deformation automorphism $\left\{\tau_{t}\right\}$ obtained as the dual of $\left\{\hat{\tau}_{t}\right\}$ is also trivial. Thus the commutative Woronowicz algebra turns out to be a commutative Kac algebra $(M, \delta, R, h)$ and the dual Woronowicz algebra is the corresponding dual Kac algebra.

Proposition 4.6. An involutive Hopf algebra, a Kac algebra and a Woronowicz algebra are the same object whenever the algebras are finite dimensional.

Proof. Throughout the proof of this statement, we assume that the algebras are finite dimensional.

We first prove that an involutive Hopf algebra $(M, \delta, \varepsilon, \kappa)$ with a faithful Haar state $h$ is a Woronowicz algebra. Let $\left\{\pi_{h}, \mathfrak{S}_{h}\right\}$ be the GNS representation. Then $\pi_{h}$ is faithful and $\mathfrak{5}_{h}$ is identified with a finite dimensional left Hilbert algebra. The linear operator $H$ on $\mathfrak{S}_{h}$ defined by $H \eta_{h}(x)=\eta_{h}\left(\kappa^{2}(x)\right)$ is positive and invertible. It also satisfies

$$
H(\xi \eta)=(H \xi)(H \eta), \quad \xi, \eta \in \mathfrak{S}_{h} .
$$

If $\lambda \in \mathbf{C} \backslash \operatorname{Sp}(H)$, then $H-\lambda$ is invertible and $(H-\lambda)^{-1} \mathfrak{S}_{h}=\mathfrak{S}_{h}$. Therefore

$$
\xi(f(H) \eta)=\frac{i}{2 \pi} \int_{\Gamma} f\left(\lambda^{-1} H\right)\left((H-\lambda)^{-1} \xi\right) \eta d \lambda
$$

for any analytic function $f$ on some neighbourhood of $\operatorname{Sp}(H)$ in C. Here we use the same argument as in the Tomita-Takesaki theory [22] with the modular operator replaced by $H$. We then find that

$$
\xi\left(H^{\alpha} \eta\right)=H^{a}\left(\left(H^{-\alpha} \xi\right) \eta\right), \quad \alpha \in \mathbf{C}, \quad \xi, \eta \in \mathfrak{S}_{h} .
$$

Thus the one parameter unitary group $\left\{H^{t}\right\}$ implements a one parameter *-automorphism group $\left\{\tau_{t}\right\}$ of $M$ such that $\pi_{h}\left(\tau_{t}(x)\right)=H^{t t} \pi_{h}(x) H^{-t t}$ for $x \in M$, which will play the role of the deformation automorphism. Using the entire analytic extension of $\left\{\tau_{t}\right\}$, we define a mapping $R$ on $M$ by $\kappa \circ \tau_{t / 2}$. Let $K$ be the 
linear operator: $\eta_{h}(x) \rightarrow \eta_{h}(\kappa(x))$. Then $K H=H K$. Hence $\kappa \circ \tau_{\alpha}=\tau_{\alpha} \circ \kappa$ for $\alpha \in \mathbf{C}$. Thus $R$ is an involutive $*$-antiautomorphism on $M$, which will play the role of the unitary antipode. Since $\sigma \circ(\kappa \otimes \kappa) \circ \delta=\delta \circ \kappa$, the Kac-Takesaki operator $W$ obtained in Remark 2.6 commutes with $H \otimes H$ as well as $H^{1 / 2} \otimes H^{1 / 2}$. Thus $\sigma \circ(R \otimes R) \circ \delta=\delta \circ R$ and $\left(\tau_{t} \otimes \tau_{t}\right) \circ \delta=\delta \circ \tau_{t}$. The $\left\{\tau_{t}\right\}$-invariance of the Haar state $h$ is clear. The strong left invariance is already verified in Remark 1.2. Thus $(M, \delta, R, \tau, h)$ is a Woronowicz algebra.

The fact that a finite dimensional Woronowicz algebra is a Kac algebra is shown in Remark 3.18.

Finally we prove that a finite dimensional Kac algebra $(M, \delta, R, h)$ is an involutive Hopf algebra with a faithful Haar state. Since a finite dimensional Kac algebra is discrete, the exists an element $\varepsilon$ in $M^{*}$ such that $\phi^{*} \varepsilon=\phi=\varepsilon^{*} \phi$ for $\phi$ in $M^{*}$. Thus $\varepsilon$ is the counit. It suffices to verify that $R$ play the role of the coinverse:

$$
\mu \circ(R \otimes \mathrm{id}) \circ \delta(a)=\mu \circ(\mathrm{id} \otimes R) \circ \delta(a)=\varepsilon(a) 1,
$$

where $\mu$ is a product in $M$, i.e. a bilinear mapping of $M \otimes M$ to $M$ with $\mu(x \otimes y)=x y$. First we notice that

$$
\hat{\eta} \otimes \hat{\eta}(\varphi \circ \mu)=V \eta_{\hat{h} \otimes \hat{h}}(\hat{\delta}(\hat{\pi}(\varphi))), \quad \varphi \in M_{*},
$$

where $V$ is the flip operator on $\mathfrak{S} \otimes \mathfrak{S}: \xi \otimes \eta \rightarrow \eta \otimes \xi$. Indeed, if $\theta_{1}$ and $\theta_{2}$ are elements in $\hat{M}_{*}$, then

$$
\begin{gathered}
\varphi \circ \mu\left(\hat{\hat{\pi}}\left(\theta_{1}\right) \otimes \hat{\hat{\pi}}\left(\theta_{2}\right)\right)=\varphi\left(\hat{\hat{\pi}}\left(\theta_{1} * \theta_{2}\right)\right)=(\varphi \circ R)\left(\hat{\pi}_{*}\left(\theta_{1} * \theta_{2}\right)\right)=\left(\theta_{1} * \theta_{2}\right)(\hat{\pi}(\varphi \circ R)) \\
=\left(\theta_{1} \otimes \theta_{2}\right)(\hat{\delta}(\hat{\pi}(\varphi \circ R)))=\left(\theta_{2} \circ \hat{R} \otimes \theta_{1} \circ \hat{R}\right)(\hat{\delta}(\hat{\pi}(\varphi)))
\end{gathered}
$$

and hence

$$
\begin{gathered}
\left((\hat{\eta} \otimes \hat{\eta})(\varphi \circ \mu) \mid \eta_{h \otimes h}\left(\hat{\hat{\pi}}\left(\theta_{1}\right)^{*} \otimes \hat{\hat{\pi}}\left(\theta_{2}\right)^{*}\right)\right)=\left((\hat{\hat{\eta}} \otimes \hat{\hat{\eta}})\left(\theta_{2} \circ \hat{R} \otimes \theta_{1} \circ \hat{R}\right) \mid \eta_{\hat{h} \otimes \hat{h}}\left(\hat{\delta}\left(\hat{\pi}(\varphi)^{*}\right)\right)\right) \\
=\left(\eta_{\hat{h} \otimes \hat{h}}\left(\hat{\delta}(\hat{\pi}(\varphi)) \mid(\hat{J} \otimes \hat{J})(\hat{\hat{\eta}} \otimes \hat{\hat{\eta}})\left(\theta_{2} \circ \hat{R} \otimes \theta_{1} \circ \hat{R}\right)\right) .\right.
\end{gathered}
$$

Since $\hat{J} \hat{\hat{\eta}}(\theta)=\hat{\hat{\eta}}\left(\theta^{*}\right)$ and $J \hat{\hat{\eta}}(\theta)=\hat{\hat{\eta}}\left(\theta^{*} \circ \hat{R}\right)$ by Corollary 3.6.2, we see that

$$
\begin{aligned}
& \left((\hat{\eta} \otimes \hat{\eta})(\varphi \circ \mu) \mid(J \otimes J) \eta_{h \otimes h}\left(\hat{\hat{\pi}}\left(\theta_{1}\right) \otimes \hat{\hat{\pi}}\left(\theta_{2}\right)\right)\right) \\
& =\left(\eta_{\hat{h} \otimes \hat{h}}\left(\hat{\delta}(\hat{\pi}(\varphi)) \mid(J \otimes J)(\hat{\hat{\eta}} \otimes \hat{\hat{\eta}})\left(\theta_{2} \otimes \theta_{1}\right)\right)\right. \\
& =\left(\eta_{\hat{h} \otimes \hat{h}}\left(\hat{\delta}(\hat{\pi}(\varphi)) \mid(J \otimes J) \eta_{h \otimes h}\left(\hat{\hat{\pi}}\left(\theta_{2}\right) \otimes \hat{\hat{\pi}}\left(\theta_{1}\right)\right)\right) .\right.
\end{aligned}
$$

Using formula (4.6), we find that

$$
\begin{aligned}
\varphi \circ \mu \circ(R \otimes \mathrm{id}) \circ \delta(a) & =\left((\hat{\eta} \otimes \hat{\eta})(\varphi \circ \mu) \mid \eta_{h \otimes h}\left((R \otimes \mathrm{id})(\delta(a))^{*}\right)\right) \\
& =\left(V \eta_{\hat{h} \otimes \hat{h}}\left(\hat{\delta}(\hat{\pi}(\varphi)) \mid(\hat{J} \otimes J) \eta_{h \otimes h}(\delta(a))\right)\right.
\end{aligned}
$$




$$
\begin{aligned}
& \left.=\left(V \hat{W} \eta_{\hat{h} \otimes \hat{h}}(1 \otimes \hat{\pi}(\varphi)) \mid(\hat{J} \otimes J) W \eta_{h \otimes h}(1 \otimes a)\right)\right) \\
& \left.=\left(W^{*} V(\hat{\eta} \otimes \hat{\eta})(\varepsilon \otimes \varphi) \mid W^{*}(\hat{J} \otimes J) \eta_{h \otimes h}(1 \otimes a)\right)\right) \\
& =\left((\hat{\eta} \otimes \hat{\eta})(\varphi \otimes \varepsilon) \mid \eta_{h \otimes h}\left(1 \otimes a^{*}\right)\right)=\varepsilon(a) \varphi(1) .
\end{aligned}
$$

Similarly, we have $\mu \circ(\mathrm{id} \otimes R) \circ \delta(a)=\varepsilon(a) 1$. Thus (4.5) holds. Hence $(M, \mu, \delta$, $\varepsilon, R)$ is an involutive Hopf algebra with a faithful Haar state $h$.

The results in [27; Appendix A2] are deduced from the above discussions.

Remark 4.7. (The uniqueness of Haar weights up to scalar) (i) Let $(M, \delta, R$, $\tau, h)$ be a Woronowicz algebra and $G=\{x \in M: \delta(x)=x \otimes x, x \neq 0\}$. If $u \in G$ satisfies $\tau_{t}(u)=u$, then $\delta\left(\sigma_{t}^{h}(u)\right)=u \otimes \sigma_{t}^{h}(u)$ by (3.4), and hence $\delta(v)=1 \otimes v$ for $v=u^{*} \sigma_{t}^{h}(u)$. Since $R(u)=u^{-1}$, we have $R(v) \otimes v=\delta(R(v) v)=1 \otimes 1$. Thus $v=1$, which means that $\sigma_{t}^{h}(u)=u$.

(ii) Let $\phi$ be another Haar weight on $(M, \delta, R, \tau)$. Since the weight $\phi \circ R$ is right invariant, it is $\left\{\sigma_{t}^{h}\right\}$-invariant by (3.4). Since $R \circ \sigma_{t}^{h}=\sigma_{-t}^{h \circ R} \circ R$ $=A d_{\rho^{t}} \circ \sigma_{-t}^{h} \circ R$, the weight $\phi$ is $\left\{\sigma_{t}^{h}\right\}$-invariant by assertion (i) replaced $h$ by $\phi$. Hence the weight $h+\phi$ commutes with the Haar weight $h$. Repeating the same argument as in [20] together with Corollary 3.11.1, we prove that $\phi$ is a scalar multiple of $h$.

\section{$\S 5$. Examples}

Quantum group $S L_{q}(2, \mathbf{C})$ (see [10] for example): Assume that $q \in \mathbf{C}(q \neq 0$, 1 ). Let $A$ be the $\mathbf{C}$-algebra generated by the four elements $x, u, v, y$ satisfying the condition of quantum matrix:

$$
x u=q u x, x v=q v x, u y=q y u, v y=q y v, u v=v u, x y-y x=\left(q-q^{-1}\right) u v
$$

and the normalization condition of the quantum determinant:

$$
x y-q u v=1 \text {. }
$$

Let $X$ be an element in $M(2, A) \cong M(2, \mathbf{C}) \otimes A$ of the form

$$
\left(\begin{array}{ll}
x & u \\
v & y
\end{array}\right) .
$$

Then the family $\left(A, \delta_{A}, \varepsilon_{A}, \kappa_{A}\right)$ is a Hopf algebra with respect to the comultiplication $\delta_{A}$, the counit $\varepsilon_{A}$ and the coinverse (antipode) $\kappa_{A}$ given by

$$
\left(\mathrm{id} \otimes \delta_{A}\right)(X)=X \dot{\otimes} X, \quad\left(\mathrm{id} \otimes \varepsilon_{A}\right)(X)=1_{\mathrm{C}^{2}}, \quad\left(\mathrm{id} \otimes \kappa_{A}\right)(X)=X^{-1},
$$


where id is the identity mapping in $M(2, \mathbf{C})$ and $\dot{\otimes}$ is the contraction tensor product given by $\left(a_{l \jmath}\right) \dot{\otimes}\left(b_{l \jmath}\right)=\left(\sum_{k=1}^{n} a_{i k} \otimes b_{k j}\right)$. The Hopf algebra is then interpreted as the coordinate ring of $S L_{q}(2, \mathbf{C})$ denoted by $A\left(S L_{q}(2, \mathbb{C})\right)$.

Quantum group $S L_{q}(n, \mathbf{C})$ for $n \geq 2$ : Assume that $q \in \mathbb{C}(q \neq 0,1)$. Let $A$ be the $\mathbf{C}$-algebra generated by $n^{2}$ matrix elements $x_{i j}(i=1, \cdots, n ; j=1, \cdots, n)$ of the quantum matrix $X$ satisfying

i) the condition of quantum matrix: Each $2 \times 2$ minor matrix

$$
\left(\begin{array}{ll}
x_{l \jmath} & x_{i l} \\
x_{k j} & x_{k l}
\end{array}\right) \quad 1 \leq i<k \leq n ; 1 \leq j<l \leq n,
$$

satisfies condition (5.1); and

ii) the normalization condition of the quantum determinant:

$$
\sum_{\sigma \in S_{n}}(-q)^{l(\sigma)} x_{1 \sigma(1)} x_{2 \sigma(2)} \cdots x_{n \sigma(n)}=1,
$$

where $S_{n}$ is the symmetric group of order $n$ and $l(\sigma)$ is the cardinality of the set $\{(i, j): i<j, \sigma(i)>\sigma(j)\}$. The left hand side is denoted by $\operatorname{det}_{q}(X)$. Then the family $\left(A, \delta_{A}, \varepsilon_{A}, \kappa_{A}\right)$ with respect to $\delta_{A}, \varepsilon_{A}, \kappa_{A}$ with (5.2) is a Hopf algebra denoted by $A\left(S L_{q}(n, \mathbf{C})\right)$, which is interpreted as the coordinate ring of $S L_{q}(n, \mathbb{C})$.

Let $R$ be the $A_{n-1}$ type solution of the Yang-Baxter equation $R_{12} R_{13} R_{23}$ $=R_{23} R_{13} R_{12}$ on $\mathrm{C}^{n} \otimes \mathrm{C}^{n}$ given by

$$
R=\sum_{i, j=1}^{n} q^{\delta_{l j}} e_{i i} \otimes e_{J j}+\left(q-q^{-1}\right) \sum_{l \supset J} e_{i j} \otimes e_{\jmath t}
$$

Then condition i) is equivalent to the equation $R X_{1} X_{2}=X_{2} X_{1} R$, where $X_{t}$ is the matrix $X$ acting on the $i$-th space of $\mathbf{C}^{n} \otimes \mathbf{C}^{n}$. Let $F$ be the flip operator $\sum_{l, j=1}^{n} e_{l j} \otimes e_{\jmath}$ on $\mathbf{C}^{n} \otimes \mathbf{C}^{n}$ and denote $F R$ by $T$. Then

$$
T=\sum_{i, J=1}^{n} q^{\delta_{l j}} e_{l j} \otimes e_{j i}+\left(q-q^{-1}\right) \sum_{i<J} e_{l i} \otimes e_{J J},
$$

and condition i) is equivalent to the equation $T X_{1} X_{2}=X_{1} X_{2} T$.

Let $\delta_{A}, \varepsilon_{A}$ and $\kappa_{A}$ be the coproduct, the counit and the antipode of $A_{q}(S L(n, \mathbf{C}))$ given by the same formulae as $(5.2)$ :

$$
\delta_{A}\left(x_{t \jmath}\right)=\sum_{k=1}^{n} x_{t k} \otimes x_{k \jmath}, \varepsilon_{A}\left(x_{i j}\right)=\delta_{i j}, \kappa_{A}\left(x_{i j}\right)=(-q)^{i-\jmath} \operatorname{det}_{q}\left(X_{j l}\right),
$$

where $X_{\mu}$ is the $(n-1) \times(n-1)$ quantum matrix obtained from the quantum matrix $X$ by deleting the $j$-th row and the $i$-th column. We sometimes identify the quantum matrix $X$ in $M(n, A)$ with an element $\sum_{l, j=1}^{n} e_{\imath \jmath} \otimes x_{\imath \jmath}$ in $M(n, \mathbb{C}) \otimes A$. Then the $n \times n$ matrix $\left(\kappa_{A}\left(x_{t}\right)\right)$ is the inverse of $X$ in $M(n, A)$. Therefore the family $\left(A, \delta_{A}, \varepsilon_{A}, \kappa_{A}\right)$ is a Hopf algebra, which we denote by $A\left(S L_{q}(n, \mathbb{C})\right)$. 
Quantum group $S U_{q}(n)$ : In what follows we restrict $q$ to be real and consider an involution in $A\left(S L_{q}(n, \mathbf{C})\right)=\left(A, \delta_{A}, \varepsilon_{A}, \kappa_{A}\right)$ defined by

$$
x_{l j}^{*}=\kappa_{A}\left(x_{l l}\right), \quad i, j \in\{1,2, \ldots, n\} .
$$

Then $A$ is a $*$-algebra. The mappings $\delta_{A}$ and $\varepsilon_{A}$ are $*$-homomorphisms and $\kappa_{A}$ is the $*$-antiautomorphism which satisfies the Woronowicz condition:

$$
\kappa_{A}\left(a^{*}\right)=\kappa_{A}^{-1}(a)^{*}, \quad a \in A .
$$

Then the involutive Hopf algebra $\left(A, \delta_{A}, \varepsilon_{A}, \kappa_{A}\right)$ is interpreted as the coordinate ring of the "quantum group $S U_{q}(n)$ ". We denote the involutive Hopf algebra by $A\left(S U_{q}(n)\right)$.

Whenever we deal with the quantum group $S U_{q}(n)$, the quantum matrix is denoted by $U$ instead of $X$. Let $U=\left(x_{t j}\right)$. The adjoint and the transpose are denoted by $U^{*}=\left(x_{j l}^{*}\right)$ and ${ }^{t} U=\left(x_{\jmath}\right)$. Then $U^{*} U=U U^{*}=1$ in $M(n, A)$. Furthermore, we have

\section{Lemma 5.1.}

(i) $\operatorname{det}_{q}(X)=\sum_{\sigma \in S_{n}}(-q)^{l(\sigma)} x_{\sigma(1) 1} x_{\sigma(2) 2} \cdots x_{\sigma(n) n}$

$$
=\sum_{\sigma \in S_{n}}(-q)^{-l(\sigma)} x_{n \sigma(n)} \cdots x_{2 \sigma(2)} x_{1 \sigma(1)} \text {. }
$$

(ii) Let $H=\sum_{J=1}^{n} q^{2 J-n-1} e_{J}$ in $M(n, \mathrm{C})(\subset M(n, A))$. Then

$$
{ }^{t} U H\left({ }^{t} U\right)^{*}=H, \quad\left({ }^{t} U\right)^{*} H^{-1}\left({ }^{t} U\right)=H^{-1} \text {. }
$$

Proof. (i) See [8] for example.

(ii) Let ${ }^{t} U=\left(y_{\imath \jmath}\right)$ with $y_{\imath \jmath}=x_{\jmath}$. Then ${ }^{t} U$ is a quantum matrix. Let $z_{l J}=(-q)^{t-\jmath} \operatorname{det}_{q}\left(\left({ }^{t} U\right)_{\jmath t}\right)$. Then the $n \times n$ matrix $\left(z_{l J}\right)$ is the inverse of ${ }^{t} U$ by (i). Since $\operatorname{det}_{q}\left({ }^{t} U\right)_{j l}=\operatorname{det}_{q}\left(U_{l \jmath}\right)$ by (i), we have

$$
z_{l \jmath}=(-q)^{1-\jmath} \operatorname{det}_{q}\left(\left({ }^{t} U\right)_{\jmath l}\right)=(-q)^{2(t-\jmath)}(-q)^{\jmath-\imath} \operatorname{det}_{q}\left(U_{l l}\right)=(-q)^{2(l-\jmath)} x_{l l}^{*},
$$

which implies that

$$
{ }^{t} U H\left(x_{i j}^{*}\right) H^{-1}={ }^{t} U\left(z_{l \jmath}\right)=1 \text { and } H\left(x_{i j}^{*}\right) H^{-1}\left({ }^{t} U\right)=\left(z_{l j}\right)\left({ }^{t} U\right)=1 .
$$

Thus ${ }^{t} U H\left({ }^{t} U\right)^{*}=H$ and $\left({ }^{t} U\right)^{*} H^{-1}\left({ }^{t} U\right)=H^{-1}$.

We will consider a representation of the *-algebra $A\left(S U_{q}(n)\right)$. Let $\mathfrak{S}^{(2)}$ $=l^{2}\left(\mathbf{Z}_{+}\right) \otimes l^{2}(\mathbf{Z})$. A representation $\left\{\pi^{(2)}, \mathfrak{S}^{(2)}\right\}$ of $A\left(S U_{q}(2)\right)$ for $q \in(0,1)$ is given by Woronowicz:

$$
\pi^{(2)}(x)=\sum_{n=0}^{\infty}\left(1-q^{2 n}\right)^{1 / 2} e_{n+1, n} \otimes 1, \pi^{(2)}(v)=\sum_{n=0}^{\infty} q^{n} e_{n, n} \otimes \sum_{m \in Z} e_{m+1, m} .
$$


Proposition 5.2. Let $U^{(n)}=\left(x_{i j}^{(n)}\right)$ be the $n \times n$ quantum matrix for $S U_{q}(n)$. Then the representation $\left\{\pi^{(n)}, \mathfrak{S}^{(n)}\right\}$ of $A\left(S U_{q}(n)\right)$ for $n \geq 2$ is given inductively by

$$
\left.\pi^{(n)}\left(U^{(n)}\right)=\left(\pi^{(2)}\left(U^{(2)}\right) \oplus 1_{n-2}\right) \dot{\otimes}\left(1 \oplus \pi^{(n-1)}\right)\left(U^{(n-1)}\right)\right) \dot{\otimes}\left(\pi^{(2)}\left(U^{(2)}\right) \oplus 1_{n-2}\right),
$$

where $\mathfrak{S}^{(n)}=\mathfrak{S}^{(2)} \otimes \mathfrak{S}^{(n-1)} \otimes \mathfrak{S}^{(2)}, \pi^{(k)}\left(U^{(k)}\right)$ denotes the $k \times k$ matrix $\left(\pi^{(k)}\left(x_{i j}^{(k)}\right)\right)$, and $\dot{\otimes}$ is the contraction tensor product.

Proof. By direst computation, it is shown that each $2 \times 2$ minor matrix of $\pi^{(n)}\left(U^{(n)}\right)$ satisfies the condition of the quantum matrix and the quantum determinant $\operatorname{det}_{q}\left(U^{(n)}\right)$ is equal to the identity 1 . The unitarity of $U^{(n)}$ is clear from the definition of the contraction tensor product.

QED

The completion of the *-algebra $A\left(S U_{q}(n)\right)$ with respect to the norm

$$
\|a\|=\sup \left\{\|\pi(a)\|: \pi \in \operatorname{Rep}\left(A\left(S U_{q}(n)\right)\right)\right\}, a \in A\left(S U_{q}(n)\right)
$$

becomes a $C^{*}$-algebra, which we denote by $C\left(S U_{q}(n)\right)$. This is nothing but a compact matrix quantum group in the sense of Woronowicz.

Let $h_{A}$ be the Haar state on $A\left(S U_{q}(n)\right)$, i.e. a normalized positive linear functional on $A\left(S U_{q}(n)\right)$ with the left invariance $\left(\mathrm{id} \otimes h_{A}\right)\left(\delta_{A}(a)\right)=h_{A}(a) 1$. The Haar state on the $C^{*}$-algebra $C\left(S U_{q}(n)\right)$ is also defined by the same condition. The existence and the uniqueness of the Haar state on a compact matrix quantum group is shown by Woronowicz [27]. The same results for $A\left(S U_{q}(n)\right)$ is given by [14] explicitly. The faithfulness of the Haar state on $C\left(S U_{q}(n)\right)$ is shown by [12] for $n=2$ and [15] for general $n \geq 2$.

Proposition 5.3. (i) There exists a *-antiautomorphism $R_{A}$ on $A\left(S U_{q}(n)\right)$ with

$$
R_{A}\left(x_{k l}\right)=(-1)^{k-l} \operatorname{det}_{q}\left(U_{l k}\right) .
$$

It satisfies $\sigma \circ\left(R_{A} \otimes R_{A}\right) \circ \delta_{A}=\delta_{A} \circ R_{A}$ and $h_{A} \circ R_{A}=h_{A}$.

(ii) There exist one parameter *-automorphism groups $\left\{\tau_{t}^{A}\right\}$ and $\left\{\sigma_{t}^{A}\right\}$ on $A\left(S U_{q}(n)\right)$ with

$$
\begin{gathered}
\tau_{t}^{A}\left(x_{k l}\right)=q^{2((k-l) t} x_{k l} \\
\sigma_{t}^{A}\left(x_{k l}\right)=q^{2 l(k+l-n-1) t} x_{k l} .
\end{gathered}
$$

Then these automorphisms satisfy the formulas

$$
\begin{array}{lll}
\left(\tau_{t}^{A} \otimes \tau_{t}^{A}\right) \circ \delta_{A}=\delta_{A} \circ \tau_{t}^{A}, & \tau_{t}^{A} \circ R_{A}=R_{A} \circ \tau_{t}^{A}, & h_{A} \circ \tau_{t}^{A}=h_{A}, \\
\left(\tau_{t}^{A} \otimes \sigma_{t}^{A}\right) \circ \delta_{A}=\delta_{A} \circ \sigma_{t}^{A}, & \sigma_{t}^{A} \circ R_{A}=R_{A} \circ \sigma_{-t}^{A}, & h_{A} \circ \sigma_{t}^{A}=h_{A} .
\end{array}
$$


Proof. (i) Let $A$ be the *-algebra $A\left(S U_{q}(n)\right)$ and $A^{\circ}$ its opposite algebra. Denote the right hand side of (5.6) by $x_{k l}^{\prime}$. Then

$$
x_{k l}^{\prime}=q^{l-k} \kappa_{A}\left(x_{k l}\right)=q^{l-k} x_{l k}^{*}
$$

by the definition of the antipode and the adjoint. It is easy to see that $x_{k l}^{\prime}$ for $k$, $l \in\{1,2, \ldots, n\}$ are the generators of $A^{\circ}$ and satisfy the same conditions as the definition of the quantum matrix and its determinant for $A^{\circ}$. Thus there exists a *-isomorphism $R_{A}$ of $A$ to $A^{\circ}$ with $R_{A}\left(x_{k l}\right)=x_{k l}^{\prime}$. This means that $R_{A}$ is a *-antiautomorphism of $A$. It suffices to verify the equality $\sigma \circ\left(R_{A} \otimes R_{A}\right) \circ \delta_{A}$ $=\delta_{A} \circ R_{A}$ only for generators, which is easy to show.

(ii) We denote by $x_{k l}^{\prime \prime}$ the right hand side of (5.7) or (5.8). Then $x_{k l}^{\prime \prime}$ for $k$, $l \in\{1,2, \ldots, n\}$ satisfy the conditions in the definition of the quantum matrix and its determinant. Thus there exists a $*$-automorphism $\tau_{t}^{A}$ or $\sigma_{t}^{A}$ on $A$ with $\tau_{t}^{A}\left(x_{k l}\right)=x_{k l}^{\prime \prime}$ or $\sigma_{t}^{A}\left(x_{k l}\right)=x_{k l}^{\prime \prime}$, respectively. By direct computation, the first two equalities of (5.9) or (5.10) are verified. For instance,

$$
\begin{gathered}
\delta_{A} \circ \sigma_{t}^{A}\left(x_{k l}\right)=q^{2 l(k+l-n-1) t} \delta_{A}\left(x_{k l}\right)=q^{2 l(k+l-n-1) t} \sum_{j=1}^{n} x_{k J} \otimes x_{J l} \\
=\sum_{j=1}^{n} q^{2 l(k-J) t} x_{k J} \otimes q^{2 i(J+l-n-1) t} x_{J l}=\left(\tau_{t}^{A} \otimes \sigma_{t}^{A}\right) \circ \delta_{A}\left(x_{k l}\right)
\end{gathered}
$$

and

$$
\begin{gathered}
\sigma_{t}^{A}\left(R_{A}\left(x_{k l}\right)\right)=q^{l-k} \sigma_{t}^{A}\left(x_{l k}\right)^{*}=q^{l-k} q^{-2 l(k+l-n-1) t} x_{l k}^{*} \\
=q^{-2 l(k+l-n-1) t} R_{A}\left(x_{k l}\right)=R_{A}\left(\sigma_{-t}^{A}\left(x_{k l}\right)\right) .
\end{gathered}
$$

It is easy to see that $h_{A} \circ \tau_{t}^{A}$ and $h_{A} \circ \sigma_{t}^{A}$ are also Haar states on $A\left(S U_{q}(n)\right)$. The uniqueness of the Haar state implies the invariance $h_{A} \circ \tau_{t}^{A}=h_{A}$ and $h_{A} \circ \sigma_{t}^{A}=h_{A}$.

QED

It will be shown that the above $\left\{\tau_{t}^{A}\right\}$ and $\left\{\sigma_{t}^{A}\right\}$ play the role of the deformation automorphism and the modular automorphism on $A\left(S U_{1 q}(n)\right)$, respectively. For each $z \in \mathbf{C}$ we define automorphisms $\tau_{z}^{A}$ and $\sigma_{z}^{A}$ by

$$
\tau_{z}^{A}\left(x_{k l}\right)=q^{2 t(k-l) z} x_{k l}, \quad \sigma_{z}^{A}\left(x_{k l}\right)=q^{2 l(k+l-n-1) z} x_{k l} .
$$

Then the antipode $\kappa_{A}$ is written in the form $\kappa_{A}=R_{A} \circ \tau_{-t / 2}^{A}=\tau_{-t / 2}^{A} \circ R_{A}$.

A unitary representation $w$ on a finite dimensional Hilbert space $\mathfrak{S}$ of a quantum group $G_{q}$ is a unitary in $\mathscr{L}(\mathfrak{S}) \otimes A\left(G_{q}\right)$ with

$$
w_{12} w_{13}=\left(\mathrm{id} \otimes \delta_{A}\right)(w), \quad(\mathrm{id} \otimes \varepsilon)(w)=1 .
$$

When $w=\sum_{l, j=1}^{m} e_{l \jmath} \otimes w_{l \jmath}$ for $m=\operatorname{dim} \mathfrak{g}$, these conditions are written in the form 


$$
\delta_{A}\left(w_{l \jmath}\right)=\sum_{k=1}^{m} w_{l k} \otimes w_{k J}, \quad \varepsilon_{A}\left(w_{l j}\right)=\delta_{l \jmath} .
$$

For two unitary representations $w$ and $w^{\prime}$ on and $a \in \mathscr{L}\left(\mathbb{E}^{\prime}\right)$ which satisfies $(a \otimes 1) w=w^{\prime}(a \otimes 1)$ is called an intertwining operator between $w$ and $w^{\prime}$. If there exists a subjective isometry as an intertwining operator, the unitary representation are said to be equivalent. Let $\hat{G}_{q}$ be the family of the equivalence classes of irreducible unitary representations of the quantum group $G_{q}$. When $G$ is a complex simple Lie group, it is shown by Rosso [19] that $\hat{G}_{q} \cong \hat{G}$. The dual object $\hat{G}$ of the Lie group $S U(n)$ is identified with the set $\left\{\left(m_{1}, \cdots, m_{n-1}\right) \in\left(\mathbb{Z}_{+}\right)^{n-1}: m_{1} \geq \cdots \geq m_{n-1} \geq 0\right\}$. Then the Peter-Weyl theorem for $S U_{q}(n)$ holds [13]:

$$
A\left(S U_{q}(n)\right)=\bigoplus_{\gamma \in \hat{G}} A(\gamma) .
$$

The irreducible unitary representation $w^{\left(\gamma_{0}\right)}$ corresponding to $\gamma_{0}=(1,0, \cdots, 0) \in \hat{G}$ is given by the fundamental unitary representation $U^{(n)}$, i.e. $U^{(n)}=w^{\left(\gamma_{0}\right)}$, and $A\left(\gamma_{0}\right)$ is the linear span of its matrix elements $x_{\imath}$ for $i, j=1, \cdots, n$.

\section{Proposition 5.4.}

(i) $h_{A}\left(x_{l \jmath}^{*} x_{k l}\right)=\delta_{t k} \delta_{\jmath l} q^{2(n-l)} /[n]$,

(ii) $h_{A}\left(x_{l \jmath} x_{k l}^{*}\right)=\delta_{l k} \delta_{\jmath l} q^{2(J-1)} /[n]$, where $[n]=\left(1-q^{2 n}\right) /\left(1-q^{2}\right)$.

Proof. By the right invariance of the Haar state $h_{A}$,

$$
h_{A}\left(x_{\imath \jmath}^{*} x_{\imath \jmath}\right) 1=\left(h_{A} \otimes \mathrm{id}\right)\left(\delta_{A}\left(x_{\imath \jmath}^{*} x_{l j}\right)\right)=\sum_{k, l=1}^{n} h_{A}\left(x_{t k}^{*} x_{t l}\right) x_{k J}^{*} x_{l \jmath} .
$$

Since $\sum_{k=1}^{n} x_{k j}^{*} x_{k \jmath}=1$, we see that

$$
\sum_{k=1}^{n}\left\{h_{A}\left(x_{t \jmath}^{*} x_{t \jmath}\right)-h_{A}\left(x_{t k}^{*} x_{t k}\right)\right\} x_{k \jmath}^{*} x_{k \jmath}=\sum_{k \neq l} h_{A}\left(x_{t k}^{*} x_{t l}\right) x_{k \jmath}^{*} x_{l \jmath}
$$

The set of elements $x_{k n}^{*} x_{l n}$ for $k, l \in\{1, \cdots, n\}$ is linearly independent by the representation in Proposition 5.2. Hence

$$
h_{A}\left(x_{t k}^{*} x_{l l}\right)=\delta_{k l} h_{A}\left(x_{l j}^{*} x_{l j}\right), \quad k, l=1, \cdots, n .
$$

Thus, the row vectors $\left\{\eta_{h_{A}}\left(x_{t k}\right): k=1, \cdots, n\right\}$ have the same norm and mutually orthogonal. From the relation for the transpose of $U$ in Lemma 5.1, we have

$$
\sum_{k=1}^{n} q^{n+1-2 k} h_{A}\left(x_{t k}^{*} x_{j k}\right)=\delta_{t \jmath} q^{n+1-2 \imath}
$$


Hence our assertion (i) holds by (5.12). Assertion (ii) is proved similarly. QED

Proposition 5.5. (i) If $a, b \in A\left(S U_{q}(n)\right)$, then $h_{A}(a b)=h_{A}\left(b \sigma_{-l}^{A}(a)\right)$ $=h_{A}\left(\sigma_{t}^{A}(b) a\right)$.

(ii) There exists a *-isomorphism $\pi$ of $A\left(S U_{q}(n)\right)$ onto a $\sigma$-weakly dense *subalgebra of a von Neumann algebra $M$ and a faithful normal state $h$ on $M$ such that $h \circ \pi=h_{A}$ and $\sigma_{t}^{h}(\pi(a))=\pi\left(\sigma_{t}^{A}(a)\right)$ for $a \in A\left(S U_{q}(n)\right)$, where $\left\{\sigma_{t}^{h}\right\}$ is the modular automorphism of $h$ on $M$.

Proof. Let $w$ and $w^{\prime}$ be irreducible unitary representations on $\mathfrak{S}$ and $\mathfrak{S}^{\prime}$. For each $b \in \mathscr{L}\left(\mathfrak{S}, \mathfrak{S}^{\prime}\right)$, the operators $c=(\mathrm{id} \otimes h)\left(w^{\prime *}(b \otimes 1) w\right)$ and $c^{\prime}=(\mathrm{id} \otimes h)$ $\left(w^{\prime}(b \otimes 1) w^{*}\right)$ are intertwining operators between $w$ and $w^{\prime}$. When $b$ is a matrix unit $e_{l \jmath}$ in $\mathscr{L}\left(\mathfrak{S}, \mathfrak{S}^{\prime}\right)$, the matrix elements of $c$ and $c^{\prime}$ are given by

$$
c_{k l}=h_{A}\left(w_{l k}^{*} w_{J l}\right) e_{k l}, \quad c_{k l}^{\prime}=h_{A}\left(w_{h l}^{\prime} w_{l \jmath}^{*}\right) e_{k l} .
$$

Thus, if $w$ and $w^{\prime}$ are not equivalent, then $h_{A}\left(w_{l j}^{\prime *} w_{k l}\right)=h_{A}\left(w_{l j}^{\prime} w_{k l}^{*}\right)=0$. Therefore

$$
h_{A}\left(x_{k l}^{*} a\right)=q^{2(k+l-n-1)} h_{A}\left(a x_{k l}^{*}\right)=h_{A}\left(a \sigma_{-l}^{A}\left(x_{k l}^{*}\right)\right), \quad a \in A\left(S U_{q}(n)\right),
$$

and hence

$$
h_{A}(a b)=h_{A}\left(b \sigma_{-l}^{A}(a)\right), \text { for } a, b \in A\left(S U_{q}(n)\right),
$$

which is (i). Let $f$ be the analytic function on $\mathbf{C}$ defined by $f(z)=h_{A}\left(\sigma_{:}^{A}(a) b\right)$ for $z \in \mathbf{C}$. Then $f(t+i)=h_{A}\left(\sigma_{t+l}^{A}(a) b\right)=h_{A}\left(b \sigma_{t}^{A}(a)\right)$. Thus $\left\{\sigma_{t}^{A}\right\}$ satisfies the KMS condition for $h_{A}$ on $A\left(S U_{q}(n)\right)$.

Let $\left\{\pi_{h_{A}}, \mathfrak{5}_{h_{A}}, \eta_{h_{A}}\right\}$ be the GNS representation of $A\left(S U_{q}(n)\right)$ with respect to the Haar state $h_{A}$ :

$$
\left(\pi_{h_{A}}(a) \eta_{h_{A}}(b) \mid \eta_{h_{A}}(c)\right)=h_{A}\left(c^{*} a b\right), \quad a, b, c \in A\left(S U_{q}(n)\right) .
$$

Let $M$ be the von Neumann algebra generated by $\pi_{h_{A}}\left(A\left(S U_{q}(n)\right)\right)$ and $h$ the normal state on $M$ defined by $h(x)=\left(x \eta_{h_{A}}(1) \mid \eta_{h_{A}}(1)\right)$ for $x \in M$. Then $h\left(\pi_{h_{A}}(a)\right)=h_{A}(a)$ for $a \in A\left(S U_{q}(n)\right)$. Since $h_{A}$ is $\left\{\sigma_{t}^{A}\right\}$-invariant, there exists a positive self-adjoint operator $\Delta$ with $\Delta^{t t} \eta_{h_{A}}(a)=\eta_{h_{A}}\left(\sigma_{t}^{A}(a)\right)$ and it satisfies $\Delta^{t t} \pi_{h_{A}}(a) \Delta^{-t t}=\pi_{h_{A}}\left(\sigma_{t}^{A}(a)\right)$ for $a \in A\left(S U_{q}(n)\right)$. Therefore the one parameter unitary group $\left\{\Delta^{t}\right\}$ implements a one parameter $*$-automorphism group $\left\{\sigma_{t}^{h}\right\}$ on $M$ such that $\sigma_{t}^{h}(x)=\Delta^{t t} x \Delta^{-t t}$ for $x \in M$. Since $\pi_{h_{A}}\left(A\left(S U_{q}(n)\right)\right)$ is $\sigma$-weakly dense in $M$, by a standard approximation procedure, the automorphism group $\left\{\sigma_{t}^{h}\right\}$ on $M$ is shown to satisfy the KMS condition on $M$. The uniqueness of the modular automorphism implies that it is the modular automorphism for $h$. Therefore $\sigma_{t}^{h}\left(\pi_{h_{A}}(a)\right)=\pi_{h_{4}}\left(\sigma_{t}^{A}(a)\right)$ for $a \in A\left(S U_{q}(n)\right)$ and the normal state $h$ is faithful on $M$. Thus $\pi_{h_{A}}$ is the desired $*$-isomorphism.

QED 
For an involutive Hopf algebra, the Kac-Takesaki operator $W$ : $\left(\eta_{h_{A}} \otimes \eta_{h_{A}}\right)(a \otimes b) \rightarrow\left(\eta_{h_{A}} \otimes \eta_{h_{A}}\right)\left(\delta_{A}(b)(a \otimes 1)\right)$ is unitary as shown in Remark 2.6. Define the coproduct $\delta$ on $M$ by the usual formula: $\delta(x)=W(1 \otimes x) W^{*}$. Then it is an extension of $\delta_{A}$, i.e., $\delta\left(\pi_{h_{A}}(a)\right)=\left(\pi_{h_{A}} \otimes \pi_{h_{A}}\right)\left(\delta_{A}(a)\right)$ for $a \in A\left(S U_{q}(n)\right)$ and satisfies the coassociativity. The left invariance of the state $h$ on $M$ is immediate form that of the Haar state $h_{A}$.

Since the deformation automorphism $\left\{\tau_{t}^{A}\right\}$ preserves the Haar state $h_{A}$, there exists a positive selfadjoint operator $H$ with $H^{i t} \eta_{h_{A}}(a)=\eta_{h_{A}}\left(\tau_{t}^{A}(a)\right)$. Define the deformation automorphism $\left\{\tau_{t}\right\}$ on $M$ by $\tau_{t}(x)=H^{t t} x H^{-t t}$. Then it is an extension of $\left\{\tau_{t}^{A}\right\}$, i.e., $\tau_{t}\left(\pi_{h_{A}}(a)\right)=\pi_{h_{A}}\left(\tau_{t}^{A}(a)\right)$. It is easy to see its commutativity with the coproduct and its invariance of the state $h$ by (5.9). By virtue of Proposition 5.3, each element of $A\left(S U_{q}(n)\right)$ is entire analytic with respect to the deformation automorphism $\left\{\tau_{t}^{A}\right\}$. Put $R_{A}=\kappa_{A} \circ \tau_{t / 2}^{A}$. Using the operator $\hat{J}$ defined by $\hat{J} \eta_{h_{A}}(a)=\eta_{h_{A}}\left(R_{A}\left(a^{*}\right)\right)$ for $a \in A\left(S U_{q}(n)\right)$, we define the unitary antipode $R$ on $M$ by $R(x)=\hat{J} x * \hat{J}$. Then it is an extension of $R_{A}$, i.e., $R\left(\pi_{h_{A}}(a)\right)=\pi_{h_{A}}\left(R_{A}(a)\right)$. It is easy to see its commutativity with the deformation automorphism, its invariance of the state $h$ and $\sigma \circ(R \otimes R) \circ \delta=\delta \circ R$. As we have already seen in Remark 1.2 that an involutive Hopf algebra satisfies the strong left invariance, we find that the family $(M, \delta, R, \tau, h)$ is a Woronowicz algebra, which we denote by $L^{\infty}\left(S U_{q}(n)\right)$. Thus we have the following:

Theorem 5.6. If $A\left(S U_{q}(n)\right)=\left(A, \delta_{A}, \varepsilon_{A}, \kappa_{A}\right)$ is an involutive Hopf algebra and $h_{A}$ is the Haar state, then $L^{\infty}\left(S U_{q}(n)\right)=(M, \delta, R, \tau, h)$ is a Woronowicz algebra which contains $A\left(S U_{q}(n)\right)$ as its $\sigma$-weakly dense *-subalgebra under the above notations.

Remark 5.7 Let $W$ be the Kac-Takesaki operator of $L^{\infty}\left(S U_{q}(n)\right)$. For each $\gamma \in \hat{G}$ with $G=S U(n)$, we denote by $w^{(\gamma)}=\left(w_{l \jmath}^{(\gamma)}\right)_{l, j \in I_{\gamma}}$ the corresponding irreducible unitary representation with $\kappa_{A}\left(w_{l \jmath}^{(\gamma)}\right)=w_{\jmath l}^{(\gamma) *}$. Then

$$
W=\sum_{\gamma \in \hat{G}} \sum_{l, k \in I_{\gamma}} \pi_{h}\left(w_{t k}^{(\gamma)}\right) \otimes v_{k l}^{(\gamma)}=\sum_{\gamma \in \hat{G}} \sum_{l, k \in I_{\gamma}} \sqrt{\frac{h\left(w_{k J}^{(\gamma)^{*}} w_{k j}^{(\gamma)}\right)}{h\left(w_{l j}^{(\gamma)^{*}} w_{l l}^{(\gamma)}\right)}} \pi_{h}\left(w_{l k}^{(\gamma)}\right) \otimes e_{k l}^{(\gamma)},
$$

where $v_{k l}^{(\gamma)} \eta_{h}\left(w_{l \jmath}^{(\gamma)}\right)=\eta_{h}\left(w_{k j}^{(\gamma)}\right)$ for any $j \in I_{\gamma}$ and $v_{k l}^{(\gamma)}=0$ on the orthogonal complement of the subspace $\sum_{j \in I_{\gamma}} \mathrm{C} \eta_{h}\left(w_{l j}^{(\gamma)}\right)$.

Quantum Lie algebra $s l_{q}(n, \mathbb{C})$ for $n \geq 2$ : Assume that $q \in \mathbb{C}(q \neq 0, \pm 1)$. The quantum universal enveloping algebra $\mathscr{U}\left(s l_{q}(n, \mathbf{C})\right)$ is the Hopf algebra $\left(\mathscr{U}, \delta_{\|}, \varepsilon_{/ /}, \kappa_{\|}\right)$consisting of a $\mathbb{C}$-algebra $\mathscr{U}$ generated by $4(n-1)$ elements $k_{J}^{+}, k_{J}^{-}, e_{J}, f$, for $j=1,2, \ldots, n-1$ satisfying the fundamental relations: 


$$
\begin{aligned}
& k_{l}^{+} k_{l}^{-}=k_{l}^{-} k_{l}^{+}=1, \quad k_{l}^{ \pm} k_{j}^{ \pm}=k_{J}^{ \pm} k_{l}^{ \pm} \quad(i \neq j), \\
& k_{l}^{+} e_{J} k_{l}^{-}=q e_{J}(i=j) ;=q^{-1 / 2} e_{J}(|i-j|=1) ;=e_{J}(|i-j|>1) \\
& k_{l}^{+} f_{J} k_{l}^{-}=q^{-1} f_{J}(i=j) ; q^{1 / 2} f_{J}(|i-j|=1) ;=f_{J}(|i-j|>1) \\
& e_{l} f_{J}-f_{J} e_{l}=\delta_{l \jmath}\left(q-q^{-1}\right)^{-1}\left(\left(k_{l}^{+}\right)^{2}-\left(k_{l}^{-}\right)^{2}\right) \\
& e_{\imath} e_{J}=e_{J} e_{\imath}, \quad f_{\imath} f_{J}=f_{J} f_{\imath}(|i-j|>1) \\
& e_{l}^{2} e_{J}-[2] e_{l} e_{J} e_{l}+e_{J} e_{t}^{2}=0, \quad f_{l}^{2} f_{J}-[2] f_{i} f_{J} f_{t}+f_{J} f_{l}^{2}=0 \quad(|i-j|=1)
\end{aligned}
$$

and the coproduct $\delta_{\|}$, the counit $\varepsilon_{\|}$, the antipode $\kappa_{\|}$with

$$
\begin{aligned}
& \delta_{\mathbb{I}}\left(k_{l}^{ \pm}\right)=k_{l}^{ \pm} \otimes k_{l}^{ \pm}, \delta_{\mathbb{I}}\left(e_{l}\right)=e_{l} \otimes k_{l}^{-}+k_{l}^{+} \otimes e_{l}, \quad \delta_{\mathbb{W}}\left(f_{l}\right)=f_{l} \otimes k_{l}^{-}+k_{l}^{+} \otimes f_{l} \\
& \varepsilon_{\text {// }}\left(k_{l}^{ \pm}\right)=1, \varepsilon_{\text {/ }}\left(e_{\imath}\right)=\varepsilon_{\text {/ }}\left(f_{l}\right)=0 \text {, } \\
& \kappa_{/ /}\left(k_{l}^{ \pm}\right)=k_{l}^{\mp}, \kappa_{/ /}\left(e_{l}\right)=-q e_{i}, \kappa_{/ /}\left(f_{l}\right)=-q^{-1} f_{l} \text {. }
\end{aligned}
$$

Quantum Lie algebra $\operatorname{su}_{q}(n)$ for $n \geq 2$ : Assume that $q \in \mathbf{R}(q \neq 0, \pm 1)$. Let's consider an involution : $\phi \rightarrow \phi^{\#}$ in $\mathscr{U}\left(s l_{q}(n, \mathbf{C})\right)=\left(\mathscr{U}, \delta_{\|}, \varepsilon_{/ /}, \kappa_{\|}\right)$satisfying

$$
\left(k_{l}^{ \pm}\right)^{\#}=k_{l}^{ \pm}, \quad e_{l}^{\#}=f_{l}, \quad f_{t}^{\#}=e_{l} .
$$

Then $\mathscr{U}$ is a $*$-algebra. The mappings $\delta_{\|}$and $\varepsilon_{\|}$are $*$-homomorphisms and the mapping $\kappa_{/}$is a $*$-antiautomorphism with the Woronowicz condition. The involutive Hopf algebra $\left(\mathscr{U}, \delta_{\|}, \varepsilon_{\|}, \kappa_{\|}\right)$is called the quantum universal enveloping algebra $\mathscr{U}\left(s u_{q}(n)\right)$ for the "quantum Lie algebra $s u_{q}(n)$ ".

The dual space of $A\left(S U_{q}(n)\right)=\left(A, \delta_{A}, \varepsilon_{A}, \kappa_{A}\right)$ becomes an involutive unital algebra with respect to the product $\phi * \psi=(\phi \otimes \psi) \circ \delta_{A}$ for $\phi, \psi \in A^{*}$, the involution $\phi^{\#}(a)=\phi^{*}\left(\kappa_{/ /}(a)\right)$, where $\phi^{*}(a)=\phi\left(a^{*}\right)$, and the identity $\varepsilon_{A}$. The quantum universal enveloping algebra $\mathscr{U}\left(s u_{q}(n)\right)$ is identified with a *subalgebra of the dual space $A\left(S U_{q}(n)\right)^{*}$ under the initial conditions

$$
\begin{gathered}
i \quad i+1 \\
\left(k_{l}^{ \pm}\left(x_{l m}\right)\right)=\operatorname{diag}\left(1, \cdots, 1, q^{ \pm 1 / 2}, q^{\mp 1 / 2}, 1, \cdots, 1\right), \\
\left(e_{l}\left(x_{l m}\right)\right)=e_{l, t+1}, \quad\left(f_{l}\left(x_{l m}\right)\right)=e_{l+1, l}
\end{gathered}
$$

for the quantum matrix $U=\left(x_{l m}\right)$ and the recurrence formulas

$$
\begin{aligned}
& k_{l}^{ \pm}(a b)=k_{l}^{ \pm}(a) k_{l}^{ \pm}(b), \\
& e_{l}(a b)=e_{l}(a) k_{l}^{-}(b)+k_{l}^{+}(a) e_{l}(b), \quad f_{l}(a b)=f_{l}(a) k_{l}^{-}(b)+k_{l}^{+}(a) f_{l}(b) .
\end{aligned}
$$

In what follows $A\left(S U_{q}(n)\right)=\left(A, \delta_{A}, \varepsilon_{A}, \kappa_{A}\right)$ together with the Haar state $h_{A}$ is embedded into the Woronowicz algebra $L^{\infty}\left(S U_{q}(n)\right)=(M, \delta, R, \tau, h)$ as a $\sigma$ weakly dense $*$-subalgebra. The underlying space $\mathfrak{S}$ is identified with the $L^{2}$ - 
space $L^{2}\left(A, h_{A}\right)$ through the identification $\eta_{h}\left(\pi_{h}(a)\right)=\eta_{h_{A}}(a)$ for $a \in A$. For each $\phi \in A^{*}$ we define two linear operators $\lambda(\phi)$ and $\rho(\phi)$ in by

$$
\lambda(\phi) \eta_{h_{A}}(a)=\left(\eta_{h_{A}} \otimes \phi\right)\left(\delta_{A}(a)\right) \text { and } \rho(\phi) \eta_{h_{A}}(a)=\left(\phi \otimes \eta_{h_{A}}\right)\left(\delta_{A}(a)\right) \text {. }
$$

It is easy to see that these operators are closable. The restriction of elements in the predual $M_{*}$ to $A\left(S U_{q}(n)\right)$ forms a weakly* dense subspace of $A\left(S U_{q}(n)\right)^{*}$.

Proposition 5.8. If $\phi \in\left(M_{*}\right)_{\tau}$, then $\rho(\phi)^{-}=\hat{\pi}(\phi \circ \kappa)$ and $\lambda(\phi)^{-}=\hat{F} \hat{\pi}\left(\phi^{*}\right) \hat{F}$.

Proof. By direct computation, we obtain the assertions.

QED

The modular properties for representations of $S U_{q}(n)$ in the sense of Woronowicz [27] are described by a family $\left\{f_{z}: z \in \mathbf{C}\right\}$ of multiplicative linear functionals on $A$ defined by $\rho\left(f_{z}\right) \subset \hat{\rho}^{z / 2}$, where $\hat{\rho}$ is the Radon-Nikodym derivative $(D \hat{h} \circ \hat{R}: D \hat{h})$, for the dual Woronowicz algebra $L^{\infty}\left(S U_{q}(n)\right)^{\wedge}$.

Proposition 5.9. Let $\mathscr{U}\left(s u_{q}(n)\right)$ be the quantum universal enveloping algebra.

(i) Let $\left\{\alpha_{1}, \cdots, \alpha_{n-1}\right\}$ be the fundamental root system for the Lie algebra su(n) and $P_{+}$the system of positive roots. For each $\alpha=m_{1} \alpha_{1}+\cdots+m_{n-1} \alpha_{n-1}$ in $P_{+}$we set $k^{\alpha}=\left(k_{1}^{+}\right)^{m_{1}} \cdots\left(k_{n-1}^{+}\right)^{m_{n}-1}$ and $k=\prod_{\alpha \in P_{+}} k^{\alpha}$. Then $k=\left(k_{1}^{+}\right)^{n-1}\left(k_{2}^{+}\right)^{2(n-2)}\left(k_{3}^{+}\right)^{3(n-3)}$ $\cdots\left(k_{n-2}^{+}\right)^{2(n-2)}\left(k_{n-1}^{+}\right)^{n-1}$.

(ii) The Radon-Nikodym derivative $\hat{\rho}=(D \hat{h} \circ \hat{R}: D \hat{h})$, for $L^{\infty}\left(S U_{q}(n)\right)^{\wedge}$ is the self-adjoint extension of the operator $\rho(k)^{-4}$.

Proof. (i) By direct computation, we obtain the assertion.

(ii) According to Lemma 2.14 together with the duality, we see that $\hat{\rho}=\hat{J} \Delta \hat{J} H$. For any element $a=x_{J_{1} l_{1}} \cdots x_{J_{m} l_{m}}$ in $A$ we set $j=j_{1}+\cdots+j_{m}$ and $l=l_{1}+\cdots+l_{m}$. Then Proposition 5.3 implies that

$$
\begin{aligned}
\hat{\rho} \eta_{h}(a) & =\hat{J} \Delta \hat{J} H \eta_{h}(a)=q^{2(J-l)} \hat{J} \Delta \hat{J} \eta_{h}(a)=q^{2(J-l)} \hat{J} \Delta \eta_{h}\left(R(a)^{*}\right) \\
& =q^{2(J-l)} q^{2(\jmath+l-m(n+1))} \hat{J} \Delta \eta_{h}\left(R(a)^{*}\right)=q^{2(2 \jmath-m(n+1))} \eta_{h}(a) .
\end{aligned}
$$

On the other hand,

$$
\rho(k) \eta_{h}(a)=(k \otimes \mathrm{id})(\delta(a))=k\left(x_{J_{1} J_{1}} \cdots x_{J_{m} J_{m}}\right) a=k\left(x_{J_{1} J_{1}}\right) \cdots k\left(x_{J_{m} J_{m}}\right) a
$$

and, by assertion (i),

$$
k\left(x_{J_{\iota} J_{l}}\right)=q^{-\left(J_{1}-1\right)\left(n-J_{l}+1\right) / 2} q^{J_{t}\left(n-J_{l}\right) / 2}=q^{-\left(2 J_{1}-n-1\right) / 2}, \quad i=1, \cdots, m .
$$

Hence $\hat{\rho} \eta_{h}(a)=\rho(k)^{-4} \eta_{h}(a)$ for $a \in A$. 
As for the dual Woronowicz algebra for $S U_{q}(n)$, we will discuss in separate publications.

\section{References}

[1] Baaj, S. and Skandalis, G., Unitaires multiplicatifs et dualité pour les produits croisés de $C^{+}-$ algèbre, (first version, 1988)

[3] Drinfeld, V. G., Quantum groups. Proc. ICM, Berkeley, 1(1986), 798-820.

[4] Enock, M. and Schwartz, J. M., Une dualité dans les algèbre de von Neumann, Bull, Soc. Math. France, Suppl. Mémoir, 44(1975), 1-144.

[5] - Sur la structure des algèbres de Kac, II, Proc. London Math, Soc., (3)41(1980), 465480 .

[6] Jimbo, M., A $q$-difference analogue of $U(\mathfrak{g})$ and the Yang-Baxter equation, Lett. Math. Phys.,10(1985), 63-69.

[7] Kac, G. I., Generalization of the group principle of duality, Soviet Math, Dokl., 2(1961), 581584 .

[8] Koelink, H. T., On quantum groups and $q$-special functions, Thesis 1991.

[9] Masuda, T., Mimachi, K., Nakagami, Y., Noumi, M. and Ueno, K., Representation of quantum groups, "Mapping of operator algebras", Proc. Japan-U.S. Joint Seminar, Univ. Pennsylvania, 1988, edited by H. Araki and R. Kadison, 119-128, Birkhäuser, Boston-Basel-Berlin, 1990.

[10] - Representations of the quantum group $S U_{q}(2)$ and the little $q$-Jacobi polynomials, $J$. Functional Analysis, 99(1991), 357-386.

[11] Masuda, T., and Nakagami, Y., An operator algebraic framework for the duality of the quantum groups, "Mathematical Physics X", Proc. AMP-91, Univ. Leipzig, 1991, edited by K. Schmüdgen, 291-295, Springer-Verlag, Berlin-Heiderberg-New York, 1992.

[12] Masuda, T., Nakagami, Y. and Watanabe, J., Non commutative differential geometry on the quantum $S U(2), \mathrm{I}$ : an algebraic viewpoint, $K$-theory, 4(1990), 157-180.

[13] Mimachi, K., Noumi, M. and Yamada, H., Zonal spherical functions on the quantum homogeneous space $S U_{q}(n-1) \backslash S U_{q}(n)$, Proc. Japan Acad., 65(1989), 169-171.

[14] - Finite dimensional representation of the quantum group $G L_{q}(n ; \mathbf{C})$ and the zonal spherical functions on $U_{q}(n-1) \backslash U_{q}(n)$, to appear in Japan. J. Math.

[15] Nagy, G., On the Haar measure of the quantum $S U(n)$ groups, Commun. Math. Phys., 153(1993), 217-228.

[16] Nakagami, Y., Takesaki duality for the crossed product by quantum groups, "Perspectives on Quantum and Non-Commutative Analysis - Past, Present and Future of Contents between Mathematics and Physics", Kyoto, 1992, edited by H. Araki, K. Itoh, I. Ojima and A. Kishimoto, Birkhäuser, 1993.

[17] Podleś, P., and Woronowicz, S. L., Quantum deformation of Lorentz group, Commun. Math. Phys., 130(1990), 381-431.

[18] Rosso, M., Comparison des groupes $S U(2)$ quantiques de Drinfeld et de Woronowicz, $C . R$. Acad. Sc. Paris, 304(1987), 323-326.

[19] - Finite dimensional representations of quantum analog of the enveloping algebra of a complex simple Lie algebra, Commun. Math. Phys., 117(1988), 581-593.

[20] Schwartz, J. M., Sur la structure des algèbres de Kac, I, J. Functional Analysis, 34(1979), 370406.

[21] Stratila, S., Voiclescue, D. and Zsidó, On the crossed products, I, II, Rev. Roum. Math. Pures Appl., 21(1976), 1411-1449; 22(1977), 83-117.

[22] Takesaki, M., Tomita's theory of modular Hilbert algebras and its applications, Lecture Notes in Math., 128(1970), 1-123, Springer-Verlag, Berlin-Heiderberg-New York.

[23] - Duality and von Neumann algebras, Lecture Notes in Math., 247(1972), 665-779, Springer-Verlag, Berlin-Heiderberg-New York. 
[24] Vainerman, L. I. and Kac, G. I., Non unimodular ring groups and Hopf-von Neumann algebras, Math, USSR Sbornik, 23(1974), 185-214.

[25] Vainerman, L. I., Correlation between compact groups and Kac algebras, preprint.

[26] Woronowicz, S. L., Twisted $S U(2)$ group. An example of a non-commutative differential calculus, Publ. RIMS, Kyoto Univ., 23(1987), 117-181.

[27] — Compact matrix pseudogroups, Commun. Math. Phys., 111(1987), 613-665.

[28] - Tannaka-Krein duality for compact matrix pseudogroups. Twisted $S U(N)$ groups, Invent. Math., 93(1988), 35-76.

[29] Yamagami, S., On unitary representations of compact quantum groups, preprint 1993. 